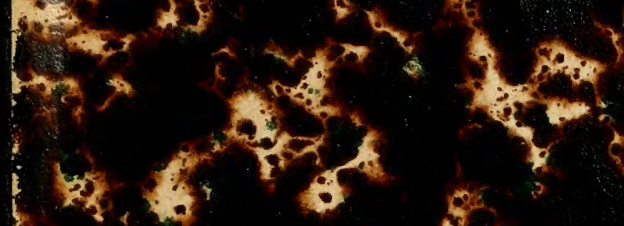

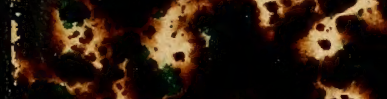

t.

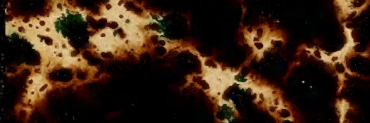

f.

t.t.

or.

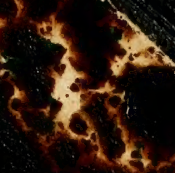

$3_{1}=$

2. 1.

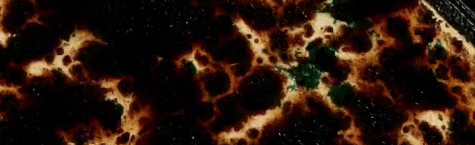

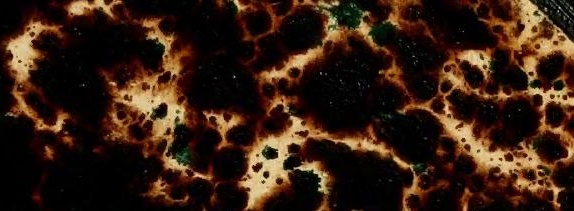

$4 \sin ^{2} \cos ^{2}$

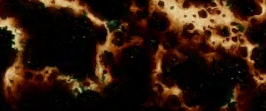

8

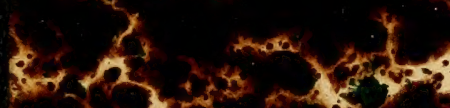

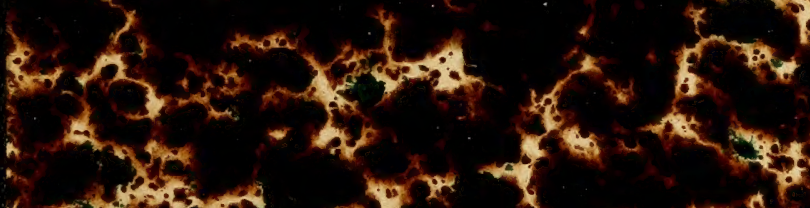

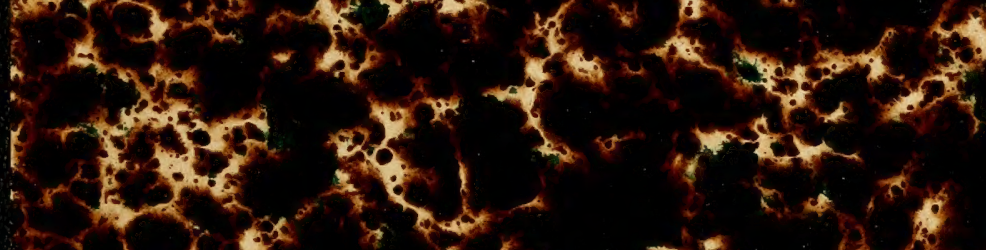

if

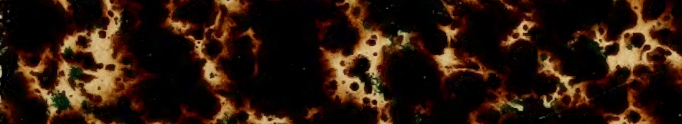

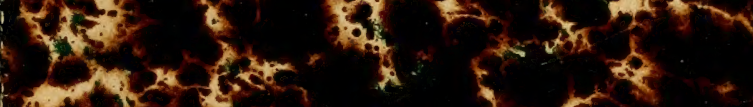

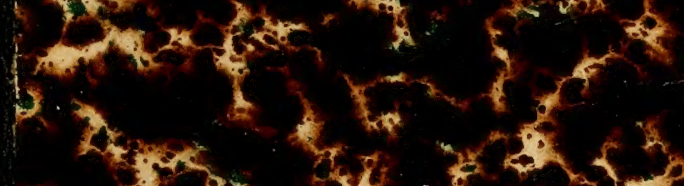

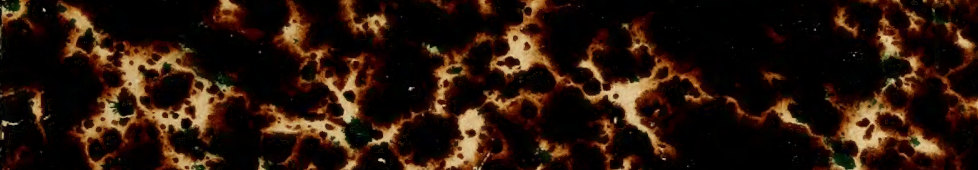

$e^{2}+x^{2}+x^{25}$

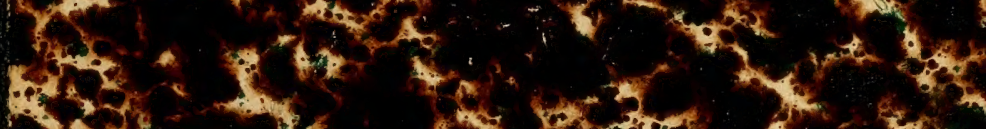

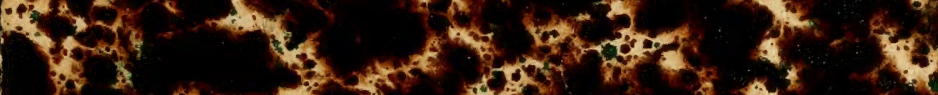
46 os to of $-x^{2}-2,+$

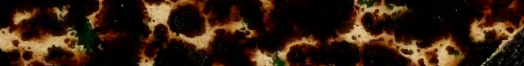




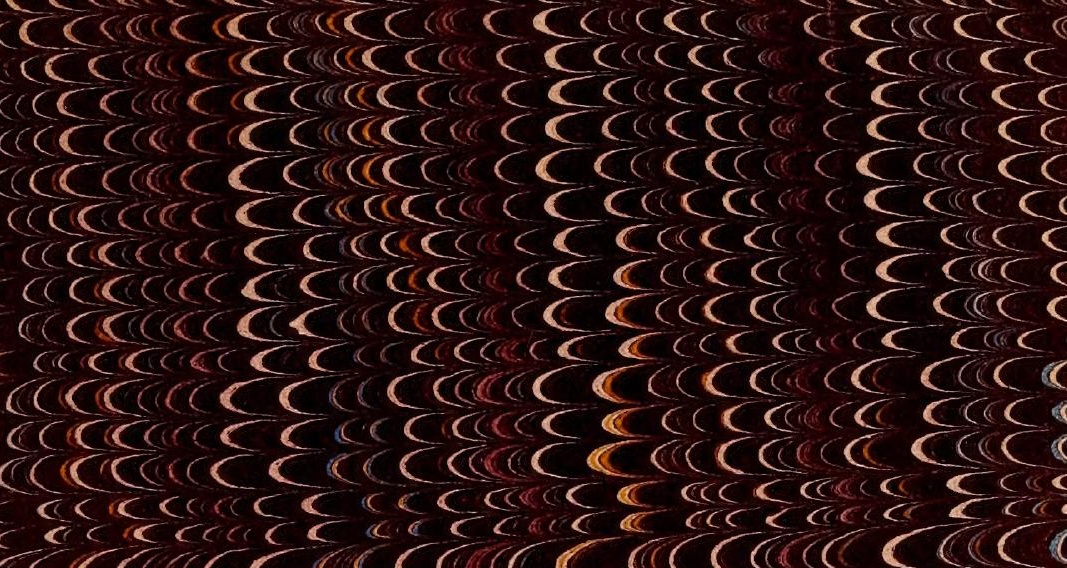

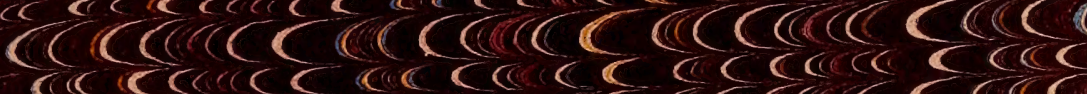

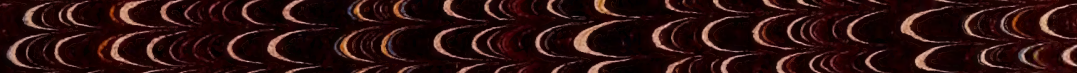

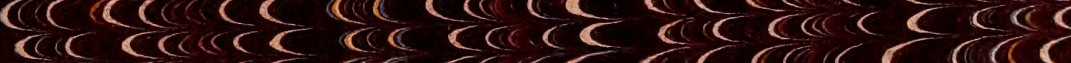

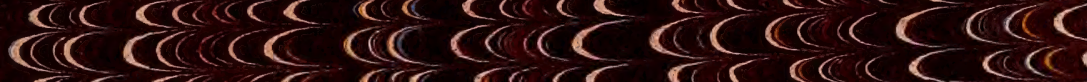

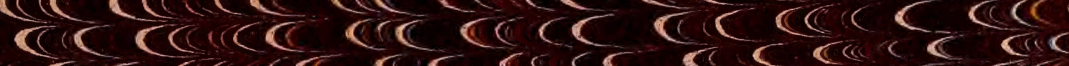

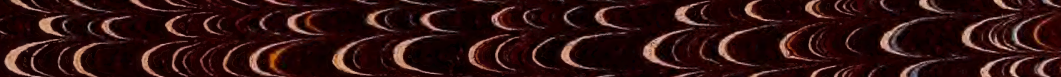

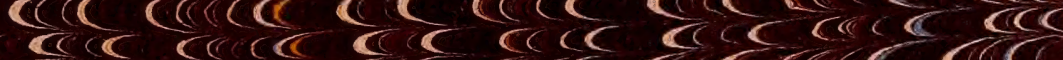

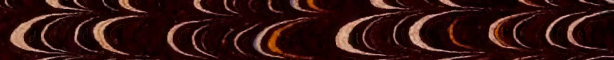

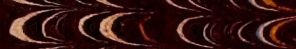
$\operatorname{coc} \cos x$

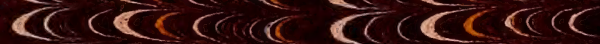

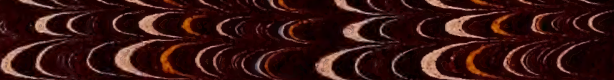

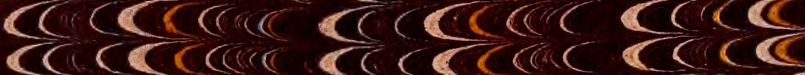

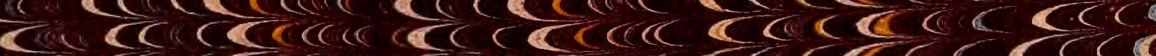

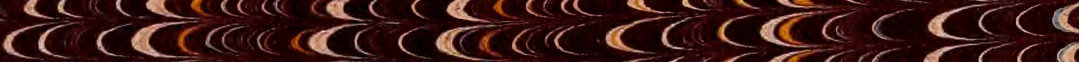

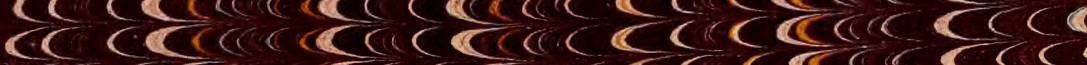

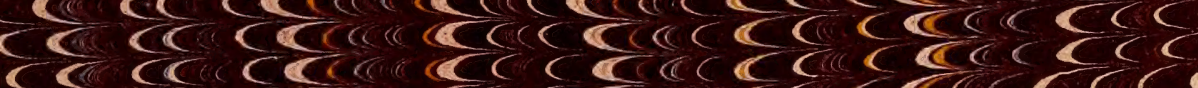

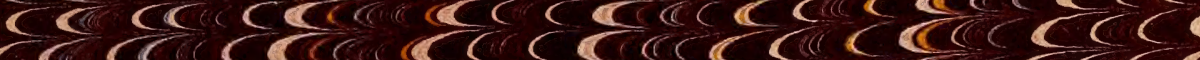

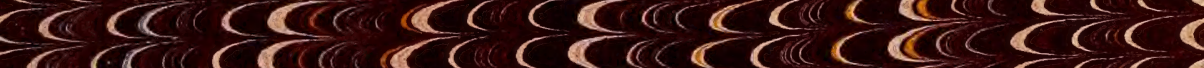

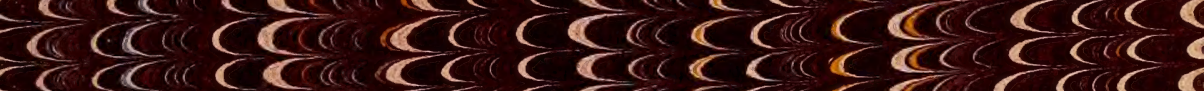

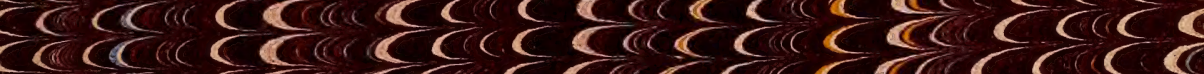

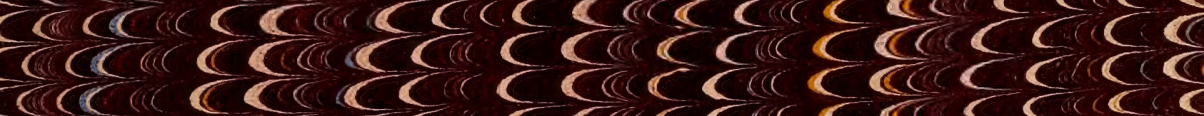
(बic (c) (C) (i) (c) बत्द त्डारी

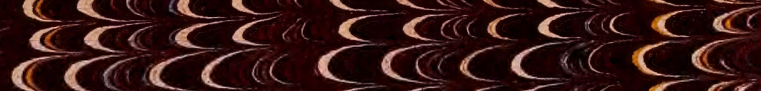
cल्तो त $<1$ CClC cc $c$

C C C CC C CC C तC तC $C$ CCC

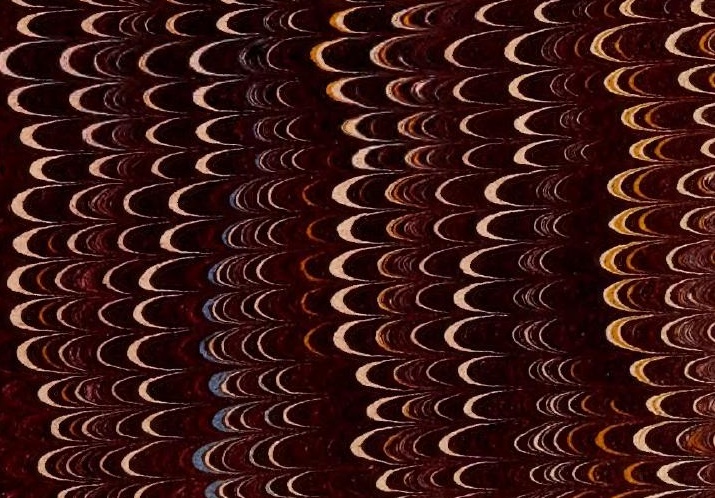



Division of Mollusks

Sectiona] ribrary 


ADOLF D'AILLY:

CONTRIBUTIONS A LA CONNAISSANCE

DES

\section{MOLLUSQUES TERRESTRES ET D'EAU DOUCE}

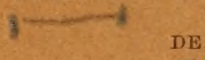

KAMEROUN.

AVEC CINQ PLANCHES.

(BIHANG T. $K$ SVENSKA VET. AKAD:S HANDL. BD. 22, AFD. IV, Nं:O 2.)

Pris 4 kr. 50 öre. 



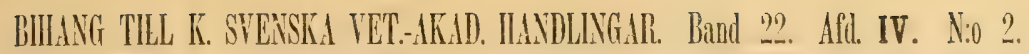

CONTRIBUTIONS A LA CONNAISSANCE

DES

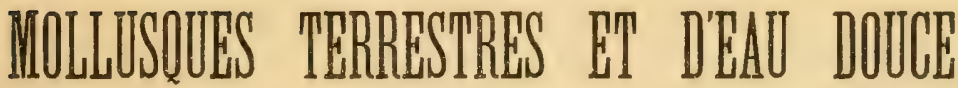

DE

\section{KAMÉROUN}

PAR

ADOLF D'AILLY

Devicion or 7 Nolles

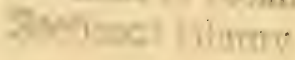

AVEC CINQ PLANCHES

COMMCXIQUÉ A LAC'ADÉME ROY. DES GCIEXCES LE 12 FÉTRIFR 1896

EXAMINÉ PAR G. LINDSTRÜM ET HJ, THÉEL

KUXGI, ROKTRYCKERIET, P. A. XORSTEDT \& SÜNER 



\section{I}

e seul mémoire qu'on rencontre, dans la littérature, spécialement consacré aux mollusques de Kaméroun, est un traité de MI. le Professem. E. vor MIartexs dans le Monatshericht der Königl. Alrademie der Wissenschaften zu Berlin, April 1876\%, sous le titre: „Die vom Prof. Dr. R. Buchnolz in Westafrika gesammelten Land- und Süsswasser-Mollusken. Outre 6 espèces provenant de la Côte d'Or, ce traité contient de Kaméroun 32 espèces terrestres, dont 17 nouvelles, et 8 espèces d'ean douce, dont une nouvelle. Le Sitzungs-Bericht der Gesellschaft naturforschender Freunde zu Berlin» des dernières amnées mentionne aussi quelques espèces de Ǩaméroun décrites par le même savant. M. le Professeur Dr. O. Boettger a de même décrit une nouvelle espèce Kamérouniemne dans le Nachricht der dentschen Malakozoologischen Gesellschaft, 1892».

Les mollusques que nous allons décrire, ont été recueillis par trois naturalistes suédois, MM. l'ingénieur P. Dusḱx, le Dr. Y. Sjöstedt et le Dr. J. R. Juxgner, qui, pendant les années 1890-1892, ont passé deux ans à voyager dans le Kaméroun. Les collections les plus riches ont été faites par les denx premiers, et nous nous faisons un devoir de reconnaître le soin exquis avec lequel ils ont su les faire. La plupart des espèces y sont représentées par des nombreux individus, et la plus grande partie en a été conservée dans l'alcool. Les notes sur les localités et sur les conditions dans lesquelles les mollusques ont été trouvés sont très précieuses. Il faut l'apprécier d'autant plus, que d'autres branches des sciences naturelles ont été l'objet essentiel de leurs traranx. Ajoutons que pendant la plus grande partie de son séjour en Afrique, M. Desśr a été occupé à mesurer et à cartograplier le 
territoire appartenant à la maison suédoise Krotson, Thatnat \& HETLBORN.

On pourra en général faire l'observation que plusieurs des expeces atioaines sont tres abondantes dans des endroits assez restreints; il en est de même a Kamérum de plusienrs especes telles que Gibbus Martensi suru, Thapsia Sjöstedti mh.. Troehozonites Lindströmi mh., Achatina iostoma PFErFfer ete.

Les espèces terrestres les plus répandues, à ce qu'il parait, sont Limicolaria Numidira (REEve), Achatina marginata Swassos, Vagimula plemroprocta (v. MARtexs), Trochozonites Ibuensis (PreIfrer) et (xibbus Liberianus (LEA). et parmi les mollusques d'eau douce, Lanistes ciliatus v. MLartexs, Melania Nigritina MIorelem et Tympanotonus fuscatus (L.).

En décrivant ces collections, nous sommes parvenu à la conriction de la grande importance fu'il faut recomnatre à la sculpture embryomnare de lacoquille comme caractère générigue, chose juspu’à présent trop négligre, si mume on trouve chez quelyues antems des allusions sur ce sujet. Pour ap)uyer cette thise, nous attirerons l'attention sur les genres 'Truchozonites, Achatina, Psendachatina, Pseuloglessula ete. Généralement on a attribué trop d"importance à la forme de la corpuille, à la grandem du dernier tour relativement à celle de la spire, au nombre des tours, et chez certaines espèces à l'échancruxe ile la columelle. Or tout cela varie beaucomp, et la sculyture, yui nous semble somvent très légirement décrite. présente sams le moindie donte des caractires de la plus haute valem. La loupe et le mirroscope n'ont-ils pas ete trop pen employés?

Une particularité que nous rencontrons souvent chez les coquilles africaines c'est l'émorme variabilite de grandem des individus adultes, notamment dans le genre Ennea.

Les collections quont apportees les trois suedois se trouvent dans le Mnsie roval d'histoire naturelle i Stockholm, dans le Mnsée zoologique de l'Eniversité d'L ${ }^{\prime}$ sal et (la plus faible partie) dans le Insée de (tothembourg; la plus grande partie de la collection conchyliologigne faite par MI. Duskis a été achetée par nous.

Avant de passer à la description de ces riches matériaux, ce nons est un devoir particulicrement agréable de présenter ¿ MLM. les Professems H.t. Théct et Ǵ. Limistriim, de Strock-

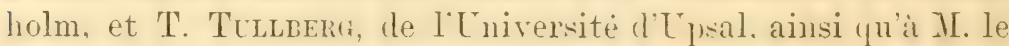


Bibliothécaire Dr. E. II. Dahlgrex de la Bibliothèque de l'Académie des Sciences, à Stockholm, notre reconnaissance respectueuse pour la bienveillance qu'ils nous ont témoignée et la libéralité avec laquelle ils ont mis à notre disposition les collections respectives.

Enfin, nous avous les obligations les plus grandes à M. le Professeur E. ros Martens pour la bonté extraordinaire avec laquelle il nous a reçu à notre visite à Berlin en vue d'étudier les énormes collections de mollusques africains du IIusée zoologique. Nous plaiguons seulement beancoup que le temps que nous arons eu à notre disposition a été trop court.

\section{Fam. STREPTAXID平.}

Genus Streptaxis. GraY.

\section{Sectio Eustreptaxis.}

\section{Streptaxis Camerunensis n. sp.}

(Pl. I, Fig. 1-5.)

Testa umbilicato-rimata, depresse ovalis, tenuis, sub-diaphana, nitida, subirisans, cerea, epidermide tenui, pallide corneo-albida incluta; spira obtusa, semiglobosa; anfr. $5^{1} 2$, primi regulares, embryonales oculo nudo levigati, sub lente præsertim infra suturam lerissime radiatim striolati et lineis spiralibus, subtilissimis, impressis, satis distantibus decussati; sequentes arcuatim confertimque costulati striisque quasi malleatis in interstitiis perspicuis spiraliter sculpti; penultimus postice prope suturam rotundato-subangulatus; ultimus antrorsum devians, antice costulatus, costis rersus basin evanescentibus, sub lente striis spiralibus linearibus densissimis subtilissime ornatus, dorso basique planulatus, lævigatus, politus, in umbilico ipso ad marginem columellarem plicatostriatus; sutura sub-canaliculata, malleato-marginata, ad aperturam leviter descendens, denique breviter ascendens; apertura lunato-ovalis, pexobliqua, pariete lamella mediocri instructa; peristoma albidum, expansiusculum, breviter reflexum marginibus conniventibus callo tenui junctis, dextero superne antrorsum arcuatim protracto. 
Diam. maj. 14-16 mill.; min. 10-11; alt. 10-11 mill.

Testa jun. perspective umbilicata, depresse semiglobosa, Irisertim basi subvaricosa; apertura lunato-subtrapezialis.

Habitat in Camerunia: Bonge (S.); Bibundi (D.).

Specimina 46, quorum 39 viv. coll.

Bien que très-voisine de S. Souleyetianus (Ретіт), qui provient des îles Séchelles, cette espèce présente néammoins des particularités qui l'en distinguent. L'espèce de M. Petrit, que nous arons eu l'occasion de voir au Musée de Berlin, est d'une conleur tirant sur le verdâtre, tandis que la notre est revêtne d'un épiderme nuance corne pâle, grisâtre, avec l'apparence de la cire. Tandis que la costulation de la première est également assez visible sur la face opposée, la seconde y est à peu près parfaitement lisse et d'un poli brillant, gravée seulement de stries spirales d'une excessive finesse, à peine perceptibles sous le microscope. Liavant-dernier des tours est moins proéminent par rapport au dernier que chez $S$. Souleyetianus, et ce n'est qu'à la fin de son développement qu'il présente un angle très léger, chose facile à constater chez les jeunes individus.

Par la taille et la forme, elle se rapproche beaucoup de s. Welwitschii Mrok, et probablement aussi de S. mangera? (GrAY), qui. tous les deux, proviement de l'Afrique occidentale, mais le premier n'étant pourru d'ancune dent à la paroi de l'ourerture, et tous les deux étant privés de la costulation de la surface, il s'en distingue fort aisément.

La dent, d'une longueur de 2 à 3 mill., située un per à droite du milien de la paroi, est comprimée, à bord aryué, un peu plus élevé vers le dehors.

L'ombilic, assez onvert et arrondi en dehors, et se terminant en derlans sur l'avant-ilemier tour par un sillon arqué, est. surtout vers le bord columellaire de l'onverture, gravé de stries plices, et quelquefois revêtu d'une callosité tris mince qui se perd sous celle de la paroi.

Streptaxis Camerumensis, qui semble être assez rare, provient des environs de la factorerie de Bonge, située an bork du Heuve Meme. On ly reneontre ca th lib, mais jamais en ahondance, sous les feuilles morter et pourries dans les fourrés sombres et humides (Buschwald). 
BIHANG TILL K. SV. VET.-AKAD. HANDL. BAND 92. AFD. IV. N:0 2. 7

\section{Genus Gibbus MontFort.}

\section{Gibbus Liberianus (LEA).}

1840 Bulimus Liberianus LEA in Philad. trans. VII. p. 457. PI. 11. Fig. 4.

1848 》 $\gg$ PFeIfFer Mon. hel. II. p. 116.

$\gg \quad \gg$ pupulus Morelet Revue zool. p. 352.

1849 Liberianus Reeve Conch. icon. Vol. V. Pl. 88, N:o 660.

$1853 \gg$ Pfeiffer Mon. hel. III. p. 359.

$\gg$ pupulus $\gg \gg \geqslant \gg 360$.

1855 Gibbus 》 Adams H. et A. Genera of rec. Mollusea p. 167.

1856 Ennea pupula Preiffer Malak. Blätter II. p. 60.

》 $\gg$ Liberiana 》 》 》 $\gg$

1858 Bulimus Liberianus Moneler Sér. Conch. p. 14.

1859 Ennea pupula PFEIFfer Mon. hel. IV. p. 336.

1860 Pupa pupula (Edentulina) Albens Heliceen ed. v. Martens p. 303.

》 $\gg$ liberiana $\gg$ 》 $\gg$ 》

1875 Ennea Liberiana H. DoHrn Mal. Bl. XXII p. 205.

$1878 \gg \quad$ 》 Jahrb. Malak. Gesellsch. p. 154.

$1881 》$ (Edentulina) Pfeiffer Nomenelator p. 18.

1885 Gibbus Liberianus (Edentulina) T'ryon Man. of Conch. Ser. 2: P. 2. p. 83, Pl. 17, Fig. 26.

1891 Ennea Liheriana E. v. Martens Sitzungsher. Gesellsch. Naturf. Freunde Berlin, Febr. 1891 p. 30

Kamérom: Etome, Itoki (D.), N'dian, Bonge, Kitta (S.), Bibundi (S., J.); (Barombi (Pr.)).

(Liberia, Gabon).

48 spée., dont 42 conservés dans l'alcool.

L'identité de cette espèce avee celle qu'a décrite et figurée MI. Deshayes (Fér. hist. II, p. 102, N:o 122, Pl. 150, Fig. 19, 20), avancée par MIM. Pfeiffer (MIon. hel. III p. 359) et MLoRELET (Sér. conch. l. c.), nous semble très douteuse, autant qu'on puisse se fier à la figure. La spire de G. Liberianus est plus acuminée; le dos du dernier tour est ventru, et la paroi de l'ouverture est horizontale, caractères yui ne se retronvent pas dans la figure mentionnée. On ne trouve également ancune trace du pli columellaire. Voilà les motifs qui nous ont forcé de la faire disparaître de la synonymie. 
La plupart des spécimens que nous avons eus sous les reux ont garde entierement on partiellement la couleur vert d'herbe de l'animal, bien qu'ils aient été conservés durant plusienu's annees dans l'alcool. En analogie de celle de $(x$. insigni: (Pferferi) et de G. Martensi Surm, la sculptures'aftaiblit sur la partie aplatie du dernier tour, située au-dessus de louverture. Chez mu seul. la fente ombilicale est agrandie presique de maniere a produire un véritable ombilic; tous les autres ont la fente caractéristique fermée et superficielle.

On trouve cette espèce non seulement parmi les racines sur le sol (I) nnx l. c.). mais rampant aussi sur les herbes it larges feuilles, (Pennisetum polystachyon Scutct.), yui atteignent la hauteur d'un homme.

\section{Gibbus insignis (PfeIffer).}

1856 Ennea insignis Pfeiffer Proc. Zool. Soc. London p. 388. Novitates Conch. I. p. 112 . T. 32 . Fig. 1, 2.

1885 Gibbus 》 (Edentulina) TrYoN Man of Conch. Ser. 2: P. 2. p. 83 Pl. 17. Fig. 16.

Kaméroun: Etome (D.), Boana (J.). (Gabon.)

3 spécimens.

Parmi plus de 100 spécimens de G. Martensi Suitu. touramasses à Etome, se trouvaient deux individus de cette rare espèce, l'un d'une longueur de 40 mill. completement déreloppé, l'autre à l'état jeune.

Ils sont revêtus d'un épiderme mince assez brillant, tirant sur l'or. Un pen plus grands que le type, ils ont anssi le test mu peu plus solide, le caractire de testa tenuis leur étant applicable, nullement celui de tenuissima.

Les figmues qui en ont été données ne sont pas bonnes: ainsi, entre autres, il faudrait que l'ourerture fût plus allongée. 


\section{Gibbus Martensi E. A. Sиптн.}

1876 Ennea insignis v. Martens Monatsber. Berlin, apr. 1876 . p. 263. T. 4, Fig. 1, 1 a.

$1878 》 \quad$ G. Pferfer Jahrb. Mal. Gesell. p. 62.

1882 Gibbus Martensi E. A. Smith Journ. of Conch. III. p. 301.

$1885 \gg \quad$ (Edentulina) I'ryon Man, of Conch. Ser, 2: P. 2. p. 83. Pl. 17. Fig. 17.

Kiaméroun: Etome (230 m. d'alt.), Bomana (670 m. d'alt.). (D.); (Victoria, Bonjongo (B)).

\section{5 spéc., dont 20 dans l'alcool.}

Nous risquerons pourtant l'assertion que la columelle est obtusement pliée d'une manière différente de celle de Gibbus insignis (Pferffer), le pli n'étant nullement denticulé. Les tours de la spire, et surtout l'avant-dernier, manifestent une tendance à se déborder mutuellement au-dessus de la suture (imbricatim) formant alors un angle obtus de lenr partie inférieure. La jemue coquille, obtusement anguleuse vers la base, est brillante an-dessous, avee des stries spirales d'mue finesse excessive; sa columelle, à bord largement réfléchi, et formant une perforation perçant toute la spire, est entièrement droite, et à son point de jonction avec le bord basal, elle forme un angle, ce qui rend l'ouverture à peu près parallélogrammique.

Les dimensions de la coquille sont très-variables: on en rencontre des individus qui ont à peine 333 mill. de longueur sur 18 de largeux, tandis que d'autres atteignent jusqu'à $40^{\circ}$ mill. sur 24. La spire compte de 6 à 7 tours. Il nous semble que les plus petits spécimens, qui sont moins ventrus et d'un déreloppement en général plus régulier, devraient être séparés sons le nom de forma minor.

Il paraît que l'espèce est restreinte à peu de localités, et elle ne se trouve probablement que dans les contrées montagneuses; habitant, comme les espèces du genre Ennea, sur le sol parmi les détritus végétaux, elle est, comme celles-là, difficile à trouver vivante, pour autant que cela ne dépend pas de ce que nos individus ont tous été recueillis pendant la saison des sécheresses. 


\section{Genus Ennea H. et A. Adars.}

\section{Sectio Uniplicaria PfeIfFer.}

\section{Ennea gemma n. sp.}

(P1. I, Fig. 28-33.)

Testa breviter rimata, cylindrica, tenuis, diaphana, cerea, albido-comea, oblique arcuatim striata; spira cylindrica apice conulum obtusum formante; anfr. 6 convexi; sutura simplici, impressa discreti, embryonales livvigati, sequentes irregulariter oblique striati, ultimus regulariter et subperpendiculariter subsuleatus, ad aperturam leviter ascendens, longitudinis ${ }^{1}$ :; fere attingens. Apertura rotundato-trigona, subverticalis, dente parietali, obliquo, compresso munita; columella ipsa in fauce in plicam compressa. Peristoma undique extus callomarginatum, vix reflexiusculum, marginibus callo aperturali, valido, filiformi junctis, dextro medio subangulatim incrassatoproducto.

Long. 4; diam. vix 2 mill.

Animal vivide corallinum.

Habitat in Camerunia (S., ubi?).

\section{Ennnea Bongeensis n. sp.}

(P1. I, Fig. 34-37.)

Testa breviter rimata, cylindrica vel whsolete obovata, solidiuscula, pellucens, cereo-albida. nitida, costulis arcuatis, rotundato-planatis ornata; spira cylindriea apice obtuso; anfr. 7 , sutura simplici, impressa discreti; primi lievigati, conulum obtusum constituentes, sequentes spiraliter, densissime, orulo nulo haud conspicue, striolati, regulariter oblique striato-costulati, costulis in ultimo, ad aperturam ascendente et ${ }^{1,3}$ longitudinis panlo superante basique dorso subgibbo, subrerticalibus, latiorilus et supra aperturam in striis mutatis vel evanescentibus. Apertura rotumdato-trigona, rerticalis. dente parietali, valiclo, compresso, obliquo, ad marginem dexterum paulum appropinunato, munita; columella ipsa profunde in fance in plicam edentulam compresia. Peristoma undique extus callo-marcinatum intusque calloso-incrassatum, marginibus eallo aperturali, 
superne filiformi junctis; dextero medio angulatim producto et impresso, tuberculo, interdum rectangulari, instructo; basali levissime reflexo; columellari expansiusculo et leviter reflexo, antrorsum arcuation producto,

Long. 61/2-71/2; diam. $3^{1 / 2}-3^{3 / 4}$ mill.

Animal pallide miniatum.

Habitat in Camerunia: Bonge (S.).

Specimina 3 exstant: 1 in coll. mus. reg. Holm., 2 in coll. mus. zool. Univers. Upsaliensis.

Les deux espèces que nous venons de décrire sont, il est vrai, très semblables entre elles dans leur apparence générale, mais le caractère de la seulpture suffira pour les distinguer. Elles se rapprochent peut-être d'E. crystallum MIorELET, mais cette derniere, que nous n'avons pas vue, appartenant au groupe de Nevillia, doit done présenter trop de différences même dans la sculpture, pour que nous risquions de trouver en elle une espèce synonyme à aucune des nôtres.

Elles ont conservé, surtont E. gemma, dans l'alcool une couleur rouge éclatante, coralline.

\section{Ennea monodon MoreleT.}

1873 Ennea monodon MIorele'r Journ. de Conch. XXI. p. 330.

$1876 \gg$ Pfeiffer Mon. hel. VII. p. 500.

conica v. Martens Monatsber. Berlin. p. 264. T. 4. Fig. 17,7 .

1878 》 $\gg$ G. Pfermer Jahrb. Malak. Gesellsch. p. 69.

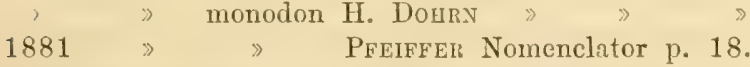

1885 \ (Uniplicaria) Trron Man. of Conch. Ser. 2: P. 2. 1. 91. Pl. 18. Fig. 62, 63.

Kaméroun: Batoki (D.), N’lian, Bonge (S.), (Bonjongo (B.), Buea (PR.));

(Liberia, Gabon).

9 spécimens, dont 6 dans l'alcool.

L'existence du tubercule à la partie la plus avancée du bord droit n'est pas mentionnée par M. Morelet, mais M. v. Mantexs l'a constatée; chez les individus que nous arons 
eus sous les penx il pase ausi d'une callosité assez légère ¿t nue dent assez distincte, comme dans les deux spécimens provenant de Buea que M. Y. Maptexs a eu la complaisance de nous domner.

La coquille, lorsqu'elle est fraîche, ne présente pas l'apparence laiteuse dont parle M. r. MARTexs; il y en a cependant des traces rhez celles qui ont éte recueillies montes ot M. Domrx (1. с.) a sams nul doute judicieusement supposé que cela dépendait de la methode de faire momrir lianimal. Comme tous ses congénères elle a été trourée sous des fenilles mortes.

\section{Ennea Dohrni E. A. SMiтh.}

1876 Ennea monodon v. Martens Monatsber. Berlin. p. 263. T. 4, Fig. 4, 5 .

1882 》 Dohrni E. A. Siıтн Journ. of Conch. III. p. 302.

1885 》 (Uniplicaria) Trron Man. of Conch. Ser. 2: P. 2. p. 91. Pl. 18. Fig. 54, 55.

Kaméroun: Buea (1.000 m. d'alt.) (J.). (Bonjongo, B.).

Un seul spécimen conservé dans l'alcool se troure au Musée d'Upsal.

Ce seul spécimen compte $6^{1 / 2}$ tours de spire; il est d'une longuenr de 13 mill. sur. $6^{1}=$ de diametre. La dent de la paroi est tres petite, presque rudimentaire. La colmmelle est rers l'intérieur assez bruspuement tronpuée. ce qui confëre à son extrémité l'aspect d'une dent aiguë à un plus haut degré que ne le signale la figure domée par M. V. MLAtrus. Nons ne serion pas surpris si cette espece, aprés l'examen attentif de plusiens individus. était déclarée identique à E. stylodon T. MIARTENS.

\section{Sectio Enneastrum Pferffer.}

\section{Ennea trigonostoma V. MARTENs.}

1876 Ennea trigonostoma v. Martens Monatsber. Berlin. p. 265. T. 4, Fig. 14-16. 
BIHANG TILL K. SV. VET.-AKAD. HANDL. BAND 22. AFD. IV. N:0 2. 13

Kamérom: Bibundi (S., J.), N'dian, Kïitta, Bonge (S.); (Bonjongo (B.), Buea (Pr.)).

23 spéc., 3 dans l'alcool.

Le bord inférieur de l'onverture ne présente pas d'angle aussi marqué que l'indique la figure donnée par M. v. Martexs; la coquille est aussi ordinairement d'une forme plus clavée. Plus la coquille diminue de longueur, plus elle devient clavée. Un seul sujet, mesurant 20 mill. de longueur sur $8^{1}$; mill. de diametre, est cylindrique, et correspond exactement en forme et en dimensions à la figure qu'a donnée M. Morelet d'Ennea pupreformis (Voy. Welw. Pl. 2. Fig. 6). Les dimensions de la coquille varient de $14^{1}=$ à 20 mill. de longueur et de $7^{1} 2$ à $8^{1}:$ mill. de diametre, et la spire compte de $8^{1}: 2$ a $9^{1} 2$ tours. Les premiers tours sont un peu imbriqués et la sculpture est plus fortement accusée vers la suture. II. Trros prétend incorrectement que les deux plis palatins de l'ourerture n'atteignent pas le bord droit; celui d'en haut aboutit an contraire an péristome, après une interruption plus on moins prononcée, et se perd dans la calloxité sur le point le plus avancé du bord droit. Le plus souvent, la région ombilicale est de même sillonnée près de la mune comprimée dn dernier tomr. Par la sculpture, qui est plutôt une costulation fine qu'une striation, cette espèce se rapproche encore d'E. pupreformis Mor. Parmi tous les E., elle semble être la plus voisine de celle-ci, dont elle diffère principalement par l'absence de la dent sur le bord droit, par le pli columellaire non dirisé, et par la forme de l'ouverture plus triangulaire.

L'espèce vit sur des plantes ainsi que sous des feuilles mortes dans les bois et sur le sol parmi des graminées (Pennisetum polystachyon).

\section{Ennea columellaris V. MARTENs.}

(P1. I, Fig. 14-18.)

1876 Ennea columellaris v. Martens Monatsber. Berlin, p. 266. T. 4. Fig. 19, 20.

1885 》 (Enneastrum) Tryon Man. of Conch. Ser. 2: P. 2, p. 94, Pl. 19, Fig. 94, 95.

Kaméroun: (S., ubi?). (Bonjongo (B.)).

Un seul individu vide se trouve au Musée de Stockholm. 
En comparant la figure que nous arous domée de cette espèce. on trouvera que la forme de notre spécimen est assez différente de celle du sujet qu'a figuré M. v. Martexs. Le nôtre est d'une forme plus ovale, et les tours de la spire sont beaucoup plus convexes, séparés par une suture assez profonde.

En décrivant l'armement de l'ouverture, MI. v. Martexs a négligé la petite dent tuberculeuse qui se trouve profondément dans l'intérieur de l'ouverture an-dessous du pli tridenté de la columelle, presque dans l'angle que forme celle-ci arec la base de la coquille. La costulation de la surface est très fortement développée relativement a la petitesse de cette belle espèce.

\section{Ennea complicata V. MARtens.}

(Pl. I, Fig. 19-21.)

1876 Ennea complicata v. Martens Monatsber. Berlin, p. 266, (nec figuræ).

$1885 \gg \quad$ (Enneastrum) TrYon Man. of Conch., Ser. 2: P. 2, p. 94 (nec fig.).

Kaméroun: Bonjongo (BuchHolz).

Quoique cette espéce n'ait été trouvée à Kaméroun par aucun des trois naturalistes Snédois, nous en arous cependant donné des figures par les motifs qu'on va lire.

Les figures que M. v. MLARtexs a jointes à sa diagnose d'E. complicata (reproduites par II. Trior) ne représentent pas cette espece, mais la suirante jusqu'a présent indécrite autant que nous en puissions juger. L'original que représentent nos figures, et que M. v. Martexs a eu la complaisance de nous donner pour comparaison, se tronvait au IInsée zoologinne de Berlin dans un tube de verre avec plusieurs individus de l'espece suivante, et ividemment un exemplaire de cette dernière espèce, 'qui à première vue ressemble heancoup à E. complicata, a éte remis an dessinateur. Or les deux dents qui, entre autres et principalement, distinguent res deux especes n'étant pas visibles sur la figme, ce n'est quiaver peine que M. V. MLritexs en la contrôlant a pu observer la faute commise.

Elle est la plus voisine d'E. trigonostoma, dont elle partage la disposition a imbriquer les premiers tours de la 
spire, et dont elle paraitrait être un diminutif. si elle ne présentait pas les particularités distinctives mentionnées par M. V. Martens.

\section{Ennea Martensi n. sp.}

1876 Ennea complicata v. MarTens Monatsber. Berlin, T. 4, Fig. 17, 18 (nec descriptio).

Testa oblique rimata, obovata, oblique arcuatim striatocostulata, cereo-albida; anfr. $7^{1}{ }^{\prime}$, primi levigati, ad suturam sub lente ruguloso-striati, cum sequentibus conulum obtusum formantes, antepenultimus et penultimus diametro decrescentes, ultimus ad aperturam haud ascendens, extus profunde hisulcatus, basi attenuatus et in carinam validain compressus. Apertura $2 / 5$ longitudinis occupans, fere verticalis, rotundatoovalis, 8-plicata: peristoma late reflexum, continum, prope marginem dextrum laminam parietalem validam, obliquam, superne triangulatim excavatam, retrorsum flexuosam profunde immittens; margine dextro superne sinuato, in sinu sat profunde tuberculo valido munito, medio antrorsum producto et flexuoso; plicæ palatales 2 oblique descendentes. antrorsum paululum divergentes, superior, marginem attingens, in margine ipso callositatem efficiens; margo columellaris edentulus, columella ipsa in fance in plicam validam, tridentatam compressa, dentibus duobus superioribus approximatis, supremo minimo, interdum eranescente; infra columellam profunde in fance dens parvus tuberculiformis.

Long. $10-10^{3 / 4}$ mill., diam. $4^{1 / 3}-5$ mill; apert. long. $3^{1 / 2-4, ~ l a t . ~ c i r c a ~} 3$ mill.

Habitat in Camerunia: Bibundi (D., S., J.); (Bonjongo (Buchноlz), Buea (Pr.)).

Spec. 13.

Pour ce qui regarde l'armement de l'ourerture, cette espèce s'accorde presque tout à fait avec E. columellaris; par l'habitus général, au contraire, elle se rapproche beancoup) d'E. complicata.

Elle diffère d'E. columellaris: par sa grandeur, par sa forme obovée très prononcée, par les tours aplatis de la 
spire, séparés par me suture peu protonde, les supérieurs en étant souvent un peu imbriqués et le nombre en comportant m de plus, par sa sculpture plus fine et plus oblique, et enfin par les dents de la columelle moins aiguës.

D'E. complicata notre espece diffire par sa forme moins cylindripue, par le sommet moins obtus de la spire, par le nombre des tours yui sont un de moins, par la costulation mu peu arquée, par la lamelle de la paroi trés oblinue, tandis yuelle est presyue perpendiculaire chez E. complicata, par' le fort repliement du bord externe, par l'ouverture relativement plus grande, et enfin, surtout par les deux dents, dont relle qui se trouve dans la simmosité du bord droit est carhée par la lamelle parictale, sur laquelle on roit d'ailleur's un tubercule correspondant, et dont l'autre, situte profondément dans l'intériemr de l'ouverture, au-dessous de la crête columellaire et un pen derriere elle, ne devient visible qu'en tom'nant la coquille un peu à gauche.

J'ai eu le plaisir de recevoir en don de M. v. MARtens denx spécimens de cette espéce sous le nom d'E. complicata. Ils proviemnent de Buea, situé sur le petit pic de Kaméroun, et s'accordent exactement avec les nôtres.

\section{Ennea perforata 11. sp.}

(Pl. I, Fig. 11-13.)

Testa anguste pervio perforata, ovata, temis, cereo-albida, nitilula. diaphana, oblinue costulata; anfl. s, couvexi, sutura warginata discreti; embryonales livves, primi jo comulum constituentes, sequentes diametro decrescentes, ultimus attenuatus, ad aperturam ascendens extuspue profunde biscrobiculatus, basi horizontalis et cirra perforationem in cristam ralidam compressus; apertura ${ }^{1}$ 3 longitudinis vecupans, stricte verticalis, rotundato sub-tetragona, 4-plicata: peristoma leviter reflexmm. continum, plicam compressam nec Hexuosam profiurle inmitens; plicar palatales 2 . supera, antrorsum fere "raneseens, in margine ipso dextero tuherculum efticiens, infera marginem non attingens: margo colnmellaris inermis, columella ipsa in fance in plicam valiolam, obliquam, edentulam compressa.

Long. $t^{1 / 2}$, diam. 2 mill.; apert. long. 11/2, lat. $1^{1 / 1}$ mill. 
Habitat in Camerunia (D., ubi?).

Specimen unicum in coll. mea exstat.

Cette petite espèce. d'me apparence très biegante. diffère de toutes ses congéneres par le sillon ombilicaire, se terminant. en une perforation d'me finesse excessive, qui perce toute la coquille, et par la forme singulière de la base du test.

\section{Ennea serrata n. sp.}

(Pl. I. Fig. 35-11.)

Testa oblique rimata. cylindrica, solidiuscula, nitida. cereoalbida; spira cylindrica apice conulum obtusum formante; anfr. \& sensim et regulariter crescentes; primi :3 convexiusculi, laevigati, sub lente subtilissime et creberrime oblique striati, tertius subtus lineis tenuissimis, oculo nudo hand conspicuis, decussatus; sequentes subplanati. infra suturam impressam. marginatam, serrato-denticulatan, plicis brevibus, obliquis, validis eleganter regulariterque ornati, ceterum sublixvigati; ultimus ad aperturam valde ascendens, basi attenuatus extusque biscrobienlatus; apertura $\rightleftharpoons$ Iongitudinis occupans, subrerticalis, rotundato-tetragona; peristoma subexpansum, anguste reflexum, continum, smperne in plican parietalem validam, intrantem, retrorsum Hexuosam complicatum, plicu palatales 2 oblique descendentes, subparallele, supera usque in marginem externum continuata et callositatem tuberculiformem ibi efficiens; margine externo supra sinuato, medio incrassato-producto et flexuoso, margine columellari dilatato, edentulo, columella ipsa in fauce in plicam subrerticalem. obtuse bidentatam compressa.

Long. 7 ; diam. $2^{2 / 3}$; apert. long. 2; lat. 2 mill.

Habitat in Camerunia (S., ubi?).

Specimen unicum in coll. mus. reg. Holmiensis exstat.

C'ette belle petite esperce se distingue assez bien de celles du même groupe par sa spire cylindrique et surtont par la sculpture près de la suture, qui en reçoit une élégante denticulation en forme de dents de scie. Il nous semble qu'elle est Ia plus roisine d'E. denticulata Mor. (Anu. Mus. Genov. III, 
1xi2, P. 202. T. 9, Fig. 10). "qui prorient de l'Abyssinie, ot d'E. elegantula (Pferffer) (Mon. hel. II. p. 351), provenant du Cap Palmas ì Liberia.

\section{Sectio Gulella. Pfeiffer.}

\section{Ennea cavidens V. MLARTENS.}

1876 Ennea cavidens v. Martens Monatsber. Berlin p. 267. T. 4, Fir. $21-23$.

$1885 \gg$ (Gulella) Tryon Man. of Conch. Ser. 2: P. 2. p. 97. Pl. 18. Fig. $72-74$.

Kaméroun: Bibundi (I.. S., J.), (Bonjongo (B.), Buea (Pr.)). (Fernando Po).

192 spécimens, dont 10 recueillis à l'état vivant.

Sur 192 spécimens, il n'y en avait que trois avec denx dents au bord inférieur de l'ourerture. Il nous semble que les sillons du dehors du dernier tomr dans la tigure domne par II. v. Martess, sont trop longs; ils ont, en effet, plutôt l'apparence d'une fossette. qui est la conséquence nécessaire de la forme de la dent correspondante.

La longueur de la eoguille varie de 10 à 12 mill.; aucun de nos sperimens n'atteint ainsi velle qu'indique M. V. MIARtex: Selom les anteurs, la famille des Streptaxida serait ovipare. mais cette espèce fait évidemment exception, comme le font anssi les streptaxis bulbulns Mor. et Michani Cr. et F. (Sér. Conch. 1. 26s, 269). Dans quatre individus recueillis au mois de juillet, il y avait des embryons. La spire en comptait 1 tour et demi, et ils mesuraient 1 mill. et demi de hanteur sur 2 mill. et demi de diam. maximum. Aplatis an sommet, ils araient la meme sculpture que la counille adulte, yui est orne d'une tine costulation (et nom graver de stries); la suture etait imprimé, et la pripipherie du dernier tour fitait "n hant fortement angulense; entin, ils étaient pourus d'un ombilic imprime. On distinguera ces embryons assez farilement sur la coquille adulte. la sculputure d'une petite partie du second tom en itant affaiblie par sa transtormation d'une costulation a une faible striation, qui marpue les limites de l'embryon. Relativement à l'espace libre entre les dents de l'ouverture. ces embryons ditaient d'une grandemr' 
BIHANG TILL K. SV. VET.-AKAD. HANDL. BAND 22. AFD. IV. N:0 2. 19

étonnante, et ce n'était qu'aves: me grande difficulté qu'on pourait les tirer de la roquille mère. Liespèce vit sur le sol parmi les hautes herbes (Pemnisetum polystachyon) et parmi les détritus végétaux. Les individus vivants sont très rares.

\section{Ennea (Gulella?) conospira V. MArtens.}

(Forma minor: Pl. I, Fig. 6-9.)

1892 Ennea conospira v. Mar'TENs Sitzungsber. Gesellsch. naturf. Freunde, Berlin, Nov, p. 182.

Kaméroun: Buea (1,000 m. d'alt.) (J.); forma minor: Kitta (D.).

Un seul spécimen de chaque forme.

Cette coquille, dont MI. v. Martens a en la bonté de me donner un type, offre une sculpture très singulière. Le premier tour de la spire est presque lisse, le second et le troisième sont ornés de stries décurrentes, filiformes, et les snivants le sont de côtes serrées et obliques, un pen irrégulierres. Sur la moitié gauche de la partie située au-dessus de l'onverture, la sculpture s'émonsse presque totalement pour se transformer, sur le reste du dernier tour, en côtes filiformes presque perpendiculaires, espacées entre elles par des intervalles réguliers trois fois plus larges que les côtes mêmes. La dent basale forme aussi une fossette sur l'extérieur de la base de l'ouverture; de plus, l'inférieure des dents columellaires 'n forme une semblable, qui se continue par un sillon superfieiel jusqu'au bout dn sillon columellaire; il en résulte que la base de la coquille se trouve comprimée en forme de crête.

La dent basale qui, dans le type principal, est saillante et comprimée des deux côtés, est clans la petite forme étendue en travers, dentelée sur le bord supérieur (ce qui toutefois peut être accidentel), et rappelle celle d'E. cavidens v. Martens, dont la dent basale est parfois bifurquée.

La forme typique, qui a été déerite par M. v. Martens d'après des spécimens qu'a rapportés M. Precss de Buea ì Kaméroun, a été recueillie par II. Juxiner en juin 1s:1, également à Buea, à $1,000 \mathrm{~m}$. d'altitude, parmi des feuilles mortes.

La sorma minor», dont nous n'avons qu'un seul individu, a été trouvée à Kitta par M. Dusśn déjà en août 1890. 


\section{Sectio Excisa n. sectio gen. Ennex.}

T'esta elongato-orata, tenuis, cerer-albida; anfr. emhryonales lavigati, serpuentes rostis ollique ad simistram abenntibus ormati; anfr. ultimus infra medium sulcatus: apertura rotmulata, plicata; peristoma reflexmm. ard insertiomem colde corisum: plica parietali oblipua, plicis palatalibus. sublorizontatibus, stricte parallelis; colnmella in fauce plicata.

\section{Ennea Duséni 11. sp.}

(Pl. I, Fig. 22-25.)

Testa oblique arenatim rimata, subelongato-ovata. temuis,

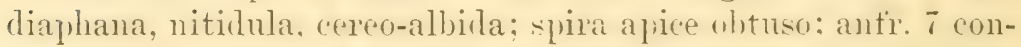
rexi, embryonales lerigati, sub lente levisime, confertim striati, sequentes costis arcuatis validis, subdistantibus, ohlique ad sinistram abeuntibus ornati, sutura impressa. marginata, denticulata discreti; ultimus basi wubghhus. prope rimam (onmpressus et cervice horizontali rotumdatu-cristatus, extus intra medium bisuleatus; apertura ": longitudinis suberpuans, rotundata, sulserticalis. fi-plicata: peristoma late reflexum. superne callo tenui, dilatato junctum, alhido-incrassatum, ad insertionem valule excisum, deinde legulariter arenatum. plica parietali ralida obliqua. intrante. haud flexuosa, plicis palatalihus :3 sublorizontalibus. stricte parallelis. translucidentihus. quarum suprema in margine ipso dextro, arl excisionem angulatim producto extusifue impresso, tulerenlum format, inferiores longiores -) extus scolbiulus efficinnt: margine colnmellari edentulo, colnmella medio plina transrersali valista, basi profundius dente parvulo instructa.

Long. 5; diam. 2; apert. long. vix 2, lat. $1^{1 / 2} / 2$ longitudo excisionis 1 mill.

Habitat in Camerunia (D., ubi?).

Specimen unicum riv. coll. in collectione mea exstat. 


\section{Ennea Boangolensis n. sp.}

(Pl. I, Fig. 26, 27.)

Testa oblique sulperforato-rimata, elongato-orata, subsolidula, diaphana, albido-cornea(?); spira elongata apice obtuso; anfr. \& convexiusculi, embryonales lrevigati, sequentes costis arenatis, validis, subdistantibus, oblique ad sinistram abeuntibus, ornati, sutura impressa, marginata, suhdenticulata discreti; ultimus basi gibbus, prope rimam compressus et cervice horizontali rotundato-cristatus, infra medium trisulcatus; apertura rotundata, subverticalis, s-plicata: peristoma late reflexum, superne callo dilatato junctum, albido-incrassatum, ad insertionem valde excisum, deinde regulariter arcuatum; plica parietali valida, obliqua, intrante, vix flexuosa; plicis palatalibus, 4 subhorizontalibus, stricte parallelis, quarum suprema in margine ipso dextro, ad excisionem angulatim producto extusque excarato, tuberculum efficit, inferiores 3 longiores approximate, in callositatem marginis dextri evanescentes, extus scrobiculos efficiunt; margine columellari edentulo; columella superne plicis duabus transversalibus, approximatis, inferiore majore, intrante, lasi profundius dente parvulo, tuberculiformi instructa.

Long. $6 \frac{1}{4}$; diam. $2^{1 / 4}$; apert. long. vix 2 , lat. $1^{1 / 2}$ mill.; longitudo excisionis $1^{1 / 2}$ mill.

Habitat in Camerunia: Boangolo (D.).

Specimen unicum in coll. mea exstat.

Au premier aspect, les deux espèces que nous venons de décrire se ressemblent infiniment, mais la denticulation de l'ouverture les distingue toutefois assez facilement l'une de l'antre. Les différences qu'offrent en outre les deux diagnoses, celle de la sculpture du sommet et celle de la coulemr et de l'éclat, dépendent de l'état de la coquille au moment de la récolte, Ennea Duséni ayant été recueillie fraîche et Ennea Boangolensis morte et décolorée.

Elles se distinguent de tontes les autres Ennea que nous connaissons par l'énorme excision du bord droit au point de l'insertion. Nous n'avons rencontré non plus chez aucune autre espèce la singulière direction de la costulation, qui, d'ailleurs, 
est tris fortement accusere. Elles se rapprochent, il est rrai, en enéréral du gronpe des Ptychotrema. mais leurs dimensions minimes et les particularites mentionnes justifieraient sans nul doute l'établissement d'une nouvelle section.

En déliant à M. l'ingénieur P. Drskex la première de ces deux espèces, toutes les deux découvertes par lui, en 1891, parui des feuilles pourries, nous rendons hommage à l'éminent royageur dont les collections ont tellement élargi notre comnaissance de la flore et de la faune de l'Afrique occidentale.

\section{Sectio Ptychotrema MIörch.}

\section{Ennea mucronata v. Martens.}

1876 Ennea mucronata v. Martexs Monatsber. Berlin p. 264 T. 4, Fig. $8-11$.

1885 Streptostele (Ptychotrema) mucronata T'RYoN Man. of Conch. S.r. 2: P. 2. 1. 110. Pl. 19. Fig. 9-11.

Kiaméroum: Boangolo, Batoki, Bomana (1).), Kitta, N'dian (S.), Buea (J.), Bibundi (D., S., J.); (Bonjongo (B.)).

257 spécimens, dont 53 recueillis à l'état vivant.

La seulpture de la corquille se compose plutôt d'une tine costulation que de stries; les tours embryomnaires sont étroitement plissés on rides, surtont an-dessons de la suture. Celle du dernier tom est distinctement marginée. I a languette de la paroi de l'ouverture dérit un demi-tour autour de l'axe de la coyuille, c'est-à-dire tont aussi loin que Jes sillons extérieur's du dernier tour, et apres a voir succesivement diminué de hantenr, elle se termine plus on moins distinctement. Les plis du fond de l'onverture montrent tous les deux, ver's leur bout droit, une élevation plus fortement accusée che\% celui d'en bas; l'écévation de ce dernier a même souvent l'apparence d'un rrai tubereule; celui d'en haut aboutit seul au péristome en s'abaissant graduellement. En dedans. la columelle est comprimée en une haute crête dont le bord est flanqué de deux dents un peutordues et comberes en arriere. La longueur de la coquille varie de 18 à 27 mill. sur $6^{1 / 2}$ à 8 mill. de 
diamètre, et la spire compte de $7^{1 / 2}$ à 9 tours. Sauf ces variations de développement, tous les spécimens que nous avons examinés se ressemblent exactement.

On la trouve sur le sol parmi les graminées ainsi que dans les bois, sous des feuilles mortes et autres détritus végétaux. Il semble que les individus frais sont assez rares ou difficiles à trouver, puisque sur un nombre si considérable, 5ỉ seulement ont été recueillis à l'état vivant. Ainsi M. SJiisTEDT nous écrit à ce sujet: Partout on rencontre sur le sol. jarmi les hautes herbes, des individus vides en grande abondance, et e'est en vain que j’ai essayé de tronver des individus vivants\%

\section{Ennea Tullbergi n. sp.}

(Pl. I, Fig. 42, 43.)

Testa oblique rimata, fusiformi-cylindrica, solidiuscula, alabastrina; spira apice papillari cylindrica, apicem versus attenuata; anfr. 9-91/2, primi 4 convexi, laves, splendentes, supra suturam sub lente subtilissime ruguloso-striati, sequentes subplanati, nitidi, costulis obliquis, superne validioribus, suturam crenulatam submarginatam efticientibus, ornati; ultimus attenuatus, cervice subcristatus, infra medium profunde hisulcatus ad aperturam leviter ascendens. Apertura subverticalis rotundato-ovalis, 5-plicata: peristoma leviter incrassatum, late reflexum, superne continuum, appressum, prope marginem dextrum, laminam validam parietalem, superne triangulatim exeavatam, retrorsum Hexuosam profunde immittens, margine dextro superne sinuato, antrorsum angulatim producto et flexuoso, superne satis profunde unituberculato, fance plicis palatalibus 2 subparallelis oblique descendentibus, quarum superior, ad marginem evanescens, in margine ipso callositatem validam constituit; columellari dilatato, edentulo. columella ipsa introrsum in plicam maximam compressa.

Long. 11/2-15 mill.; diam. 3-41/3 mill.

Habitat in Camerunia: Batoki (D.); Bibundi, Bong'e, N'llian, Itoki (S.).

Specimina 20 exstant, omnia viventia collecta.

Cette coquille est évidemment très voisine d'Ennea mucronata r. MnRtexs, et an premier coup d'oeil elle pourrait 
itles considerée comme m diminutif de rette esperee si elle ne présentait des raractères qui l'en distinguent assez rlairement anssi hien une de toutes les antres du même groupe.

La partie superieure de la spire est plus cylindrique que le reste de la coquille, et grâne an manque de toute sculpture, rauf des traces de stries ruguenses au-dessus de la suture. a peine perceptilles a la loupe, elle y devient resplendissante. Chez la précédente, au contraire, dont l'apex est plus armunié, les premiers tours sont totalement couverts dime striation plus fortement arcusée an-dessous de la suture, pet ne présentent pas non plus de développement cylindrique différant du reste du test.

Ia petite dent tubereuleuse placee, chez E. mucronata. sur le hord externe en face de la concavité de la languette parietale, est. chez E. Tullbergi, plarée plus profondément sous la rallosité du bord droit. vis-a-vis de la convexite de la languette. et quelquetois elle a l'apparence d'une très potite lamelle. Enfin, chez la dernière. le pli de la columelle est dirigé en arriere, et son hord calleux et quelquefois un peu ondule, est privé des deux dents aiguës qui caractérisent la premiere.

Les petits individus sont entièrement cylindriques.

La coquille fraiche demi-transparente, et, a l'exception des premier's tours mentionnés ci-dessus, ornée d'une costulation tine et réguliere, légerement arquée, est d'une apparence vitrée, blanchâtre.

Liespèce qui vit dans les mêmes conditions que les autres du genre Enmea, a ité dérouverte, en 18:10, par M. P. Dr'śn, qui l'a trouvée en compagnie de la précédente it Batuli sons des feuilles mortes.

\section{Ennea Buchholzi v. MARTENS.}

1876 Ennea Buchholzi v. Martens Monatsber. Berlin, p. 265. 'I'. 4, Fin. 12, 13.

1885 Streptostele (Ptychotrema) Bonjongoensis TrYos Man. of Conch. Ser 2: P. 2. 1) 110. Pl. 1!, Figr. 5, 6.

Kaneroun: Batoki (I).); Nilian, Bonge (S.); Bibundi (D., S., J.); (Bonjongo (B.); Buea (Pr.)).

25 spécimens, dont 6 recueillis à l'état vivant. 
Quant à la denticulation de l'ouverture, nous roudrions hien ajonter quelyues mots à la description de l'éminent conchyliologiste. Le pli de la paroi ne se perd pas dans l'intérieur de l'ouverture comme whez E. mucronata v. Martexs. Le bord extérieur du péristome a toujours dans l'angle supérieur, immédiatement avant sa jonction arec le pli pariétal, un tubercule, peu saillant mais distinct, qui correspond à un tubereule pareil sur la languette pariétale; viemnent ensuite un pli oblique, à borl supérieur un pen concave, non un tubereule, et enfin les deux grands plis, qui toutefois devraient être plutôt considérés comme un seul pli bifurqué, ru qu’ils sont représentés extérieurement par un seul sillon. L'inférieur en est plus long et plus saillant. Le pli inférieur du bord colnmellaire a l'apparence d'une lamelle, les deux supérieurs celle des dents. Les tours embryonnaires de la spire sont lisses et brillants à l'œil nu, tandis que sous le microscope ils sont gravés de stries d'une extrême finesse.

Le test est en outre plutôt orné de fines costules que de stries, et la forme de nos sujets est plus eylindrique que ne l'indique la figure qu'en a donnée M. v. Martens.

La longueur varie en général entre 10 et 12 mill., les sujets que nous avons devant nous étant ainsi plus petits que le type de M. V. Martens, mais un seul atteint l'énorme longueur de 18 mill. sur 5 de diametre. Sur le bord columellaire de ce sujet, entre les deux dents supérieures, se trouve un grand tubercule qui s'approche beaucoup du bord. Il nous semble qu'on en voit des traces aussi chez les autres.

Cette espèce vit dans les mêmes conditions qu'E. mu(ronata, avec laquelle on la trouve, mais elle est beancoup) plus rare.

\section{Genus Streptostele H. DoHrs.}

\section{Streptostele Buchholzi v. MARTENS.}

1876 Streptostele Buchholzi v. Martens Monatsber. Berlin, p. 262. T. 3, Fig. 16,17 . p. 108. Pl. 20, Fig. 76 .

Kaméroun: Kumbe (D.), N'dian, Bonge (S.); Bibundi (D., S.), Boana (J.); (Bonjongo (B.), Buea (Pr.)). 
64 spécimens, dont 50 recueillis frais.

A la loupe, on aperçoit entre les costules des traces de stries décurrentes du meme caractire que celles du genre Gibbus, et comme chez ce genue la sculpture s'émousse aussi sur la partie de la corpuille pui est située au-dessus de l'ourerture. Les tours embryonnaires sont lisses, et la sculpture des tours suivants est heaucoup moins marquee sur leur moitip inférieure. Pour ce qui concerne la suture, nous voudrions hien ajouter quelques mots à la diagnose: sutura impressa. minutissime, eleganter crennlata, plus minusve distincte marginata, ad aperturam leviter ascendens\% Le bord droit de lonverture est un peu aplati vers le haut, et sa partie prolongée est située plus haut rers l'insertion que ne lindique la tigure 17, domuée par M. r. Martexs. La direction de la colmmelle est assez variable; par la colmmelle qui, vers le bas. présente une déviation très considerable à ganche, les un rappellent str. Folini (Mor.), les autres se rapprochent par la columelle descendant verticalement de Str. subangusta v. MIARTENs (Sitzungsber. Naturf. Fremde 1891, p. 30). Chez quelyues exemplaires, on aperoit sur le bord interieur de la columelle une petite dent tuberuleuse. Une singularité non mentionnée jusqu'à présent, est la déviation à droite du sommet de la spire che\% plusieurs individus, résultant probahlement de leur subordination a la famille des streptaxida. Ta spire compte jusqua 10 toms, et la longueur de la corpuille varie de 12 a $16^{\prime \prime}, 4$ mill. On la trouve dans les prés ainsi que dans les furets vierges et humides, sous des feuilles mortes arec Subulina angustior Dorrr.

Streptostele pusilla n. sp.

Testa inperforata. turrita, tenuis, albida, subpellucida, nitida, sub lente subtilissime perpendiculariter striolata; spira sensim attenuata apice acuminato; anfr. $7^{1} 2$ parum convexi. sutura haud crenulata nee marginata discreti, varicibus validis hic illic ornati; apertura subobliqua, tetragono-ovata; columella vix torta, rerticalis, usifue ad basin descendens angulumpue distinctum cum margine hasali formans; peristoma rectum, leviter calloso-limbatum margine dextro modices arcuatim producto, basali paululum recedente. 
BIHANG TILL K. SV. VET.-AKAD. HANDL. BAND 22. AFD. IV. N:0 2. 27

Long. $4^{3 / 4}$ mill.; diam. $1^{1 / 2}$ apert. long. $1^{1 / 3}$; lat. 1 mill. Animal miniatum.

Habitat in Camerunia: Itoki $(1891$, S.).

Specimina duo, viventia collecta, in mus. reg. Holm. exstant.

Au premier aspect, on serait disposé à considérer cette espèce lilliputienne comme l'état jurénile de la précédente. mais i l'aide de la loupe, on reconnaîtra bientôt que c'est une espèce nouvelle assez bien caractérisée.

Sur une longueur de $4^{3}+$ mill. seulement elle compte $7^{1}$ ? tours, c'est-à-dire le même nombre que Str. Buchholzi dans l'état parfaitement développé. Elle est, en outre, d'une taille plus étroite, à sommet beaucoup plus acuminé. La suture n'est ni crénelée ni marginée. La sculpture du test est de même assez différente. Fille est tout à fait superficielle, d'une apparence ruguense, formée de stries irrégulières extrêmement fines, souvent fourchées, communiquant les unes avec les autres, et enfin on y aperçoit çà et là des varices très fortement accusées et également distantes entre elles. L'angle que forme la colmmelle à son point de jonction avec le bord basal est assez distinct, et le bord droit de l'ourerture est faiblement épaissi.

\section{Farn. VITRINID王。}

\section{Genus Urocyclus Gray.}

Urocyclus Buchholzi r. MARTENS.

1876 Urocyclus Buchholzi v. Martens Monatsber. Berlin p. 269, T. 5. Fig. 1.

'́ryon Man. of Conch. Ser. 2: P. 3. p. 163. Pl. 36, Fig. 52 .

Kaméroun: Buea (D., J.); Itoki Na N'Grolo (S.).

(Côte d'Or: Aburi (B.).)

4 spécimens. 
Quoinue cette espere ne soit commme jusinn'a présent que par un dessin firit par II. Brchuorz et reproduit par II. v. Martens (l. c.), il nous semble que les 4 spécimens que noms avons soms les venx r appartiennent. Conservés dans lalcool, ils montrent en tout cas ]a roloration quindigue res. ressin selon M. V. Martens.

Sur m fond d'me munce verdatre. on apereoit des taches hlanchâtres a hord distinct, et, à la différence de celles de loriginal de X. Bucmuocz, plus nombrenses sur le pied que sur le bouclier.

Ta partie centrale du disyue locomoteur semble igaler le tiers de la largeur totale.

Le bouclier, à bord antérieur très libre, est percé, tont prés de son extrémité postériemre tris olotusement angulense, d'un très petit pore situé justement an-dessus du nucléus de la limacelle parfaitement cachée. Relativement an développe. ment de l'animal, ce mdiment testacé est assez petit, ne mesmant que $s$ mill. de longueur sur 5 mill. de largeur chez un sprérmen, conservé dans l'alcool. de 53 mill. de longuem. Il est d'me forme elliptinue, aplatie, s'élevant graduellement vers le nucléus, qui se tronve an milien du bord posté. rieur très obtusement anguleux. La surfare supérieure est gravée de stries daccroissement inegales, et à la lompe on apercoit ad et lia des traces d'me striation ravonnant dn nuclés. Fille est, en ontre, couverte d'm épilerme mince, d'me couleur fauve tirant sur le verdâtre, et dépassant les bords du test. d'ou résulte me sorte de bordure membraneuse. Ise rôté opposé est quelque peu concave é épaissi par une faible areumulation de substance ralcaire qui. dans le tiers postriemr correspondant an muléus. ou sionsère le muscle yui le tixe au brumlier, presente des processus irréguliers, dentiformes. L'espèce semble être assez rare.

\section{Genus Aspidelus Morelet.}

\section{Aspidelus Chaperi MoreLET.}

1883 Aspidelus Chaperi Moreles, Journ. de Conch., Vol. XXXI, p. 395, Pl. 10 , Fig. 1.

1885 » Tryon Man. of Conch. Ser. 2: P. 3, p. 167, i'. :36. Fin. 57. 58. 
Kaméroun: Bibundi, Bonge, N'dian, Itoki Na N'Golo (S.). (Guinea: Assiniam (Chaper).)

\section{7 spécimens.}

Nous ne sommes pas absolument convaincu que les spécimens qui se tronvent devant nous appartiennent en effet à l'espèce de M. Monelet. Mais les particularités qu'ils présentent ne sont pas d'une importance telle, qu'elles autorisent la création d'me nouvelle espèce, d'autant plus que la diagnose de l'illustre savant se fonde sur un seul sujet.

La granulation est plus fortement accusée sur le bonclier, à bord antérieur assez libre, et le reste de l'animal est an surplus gravé d'un système réticulé de sillons d'une nuance foncée, dont ceux des cotés du corps sont obliques et prédominants. La couleur est plus foncée sur le houclier, sur le dos et sur l'extrémité du corps. La nuque est ornée de deux bandes foncées s'etendant en arrière des tentacules. Le bord de la carène du corps est pâle et unicolore. tandis que celui du disque locomoteur est orné d'une série de petites taches d'un brun foncé, rangées presque deux ì deux, également distantes entre elles et divisées en long par le sillon bien marqué qui borde le disque.

Le nucléus du rudiment testacé s'élève en faron d'ongle. Outre les stries distinctes d'accroissement, le test est grave de stries rayonnant du nucléus. Il est revêtu d'un épiderme très mince, qui se détache fort aisément. d'une couleur faure beaucoup plus fonrée que ne le montre la tigure de M. Morelex, et d'une apparence irisée sur le mudéus même. Il ne paraît pas que ce test soit aussi mince que l'indique ce savant, la partie intérieure étant légèrement fortitiée par un faible revêtement de substance calcaire. L'espece a été recueillie sur Pennisetum polystachyon aver Vaginula. Zonitarion, les Helicarion etc. 


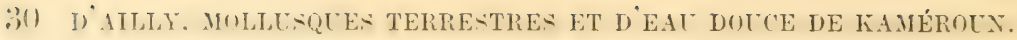

\section{Genus Helicarion Férussac.}

\section{Sectio Africarion Gudwin-Austen.}

\section{Helicarion plicatulus V. Martens.}

1876 Helicarion plicatulus v. Marress Monatsber. Berlin p. 254, 'T. 1, Fig. $5-8$.

$1885 \Rightarrow$ Thion Man. of Conch. Ser. 2: P. 3, p. 184, Pl. 43, Fig. 49-51.

Kaméroun: Bomana (D.), Buea (D., J.).

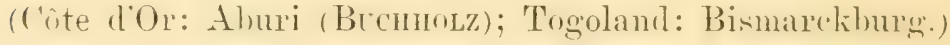

118 spécimens, dont 90 conservés dans l'alcool.

Le tom embryomaire ne montre chez cette espece ancunte trace de sculpture spirale. Nons arons entre les mains quelynes individus d'me nuance brune, et d'autres d'un éclat mat d'apparence soyense, ot qui présentent sur le dernier tour des traces dine soulpture spirale particulierement indistincte; it tmus antres égards ils śacordent exactement avec le typer.

Comme plusieur's autres especies de cette famille. Helicarion plicatulus monte ì une hantemr considérable au-dessus du niveau de la mer. Ainsi ML. Dusḱx a trouvé au-dessus de Bura. sur le grand pic de Kanérom, un individu rampant sur le sol d'une grotte à 2,500 mètres d'altitude.

L'espèce vit sur les fenilles des plantes.

\section{Helicarion pertenuis n. sp.}

(Pl. I, fig. 49-5.t.)

'Testa depresse “onvexiuscula. membranacea. irregulariter' plicatr-striata, nitida, pallide succinea, apice margaritareo, viresente; spira paulum prominula; anf. 3 , sutura adnata. vix marginata discreti; embryonalis reticulatim punctatus, ultimus celeriter crescens. powultimum fere tegens; apertur: emarginato-rotundata, paulum latios quam altior, margine supero sensim descendente. arcuato, infero leviter arenato, columellari brevissime reflexo. 6 mill.

Diam. maj. 10 ; min. $7^{1 / 2}$; alt. $6^{1 / 2}$; apert. lat. $6^{1 / 2}$; alt. 
Animal pustulatum, unicolor rufescens vel superne saturatins, interdum maculis fuscis, parvulis sursum adspersum. Pes margine emaculato subtus unicolor. tripartitus, partibus lateralibus transversim suleatis.

Habitat in Camerunia: Kitta; prope Vevoka (S.).

specimina sex in coll. mus. reg. Holm. exstant, omnia viv. collecta.

Helicarion pertenuis se distingue par son test extrêmement mince, presque membraneux, d'une couleur d'ambre jaune tirant sur le vert. La sculpture embryonnaire consiste en points microscopiques rangés en spirale et donnant à l'apex une apparence l'une élégance toute particulière.

L'animal est entierement couvert de petites papilles régrulieres, également saillantes, très serrées sur les lobes du manteau et y produisant une apparence granuleuse.

Il est le plus souvent d'une seule couleur rouge brun, un pen plus foncée vers le dos et surtont vers l'extrémité du pied; quelquefois ces parties, ainsi que les lobes, sont d'me teinte noirâtre. Le bord du disque locomoteur de tous nos spécimens est pâle, unicolore.

Les lobes, dont celui de droite couvre l'apex de la coquille. s'y étendent sur une plus grande région que chez les espèces suivantes.

\section{Helicarion columellaris $\mathrm{n}$. sp.}

(Pl. II, fig. 1-8.)

Testa depresse convexa, tenuis, irregulariter radiatim plicato-striata, nitida, straminea, apice margaritaceo; spira prominula, apice submammillato; anfr. 3 sutura adnata, marginata discreti; embryonalis reticulatim spiraliterque pulcherrime punctatus, ultimus subplanatus, basi convexior, rapide crescens, promltimm oblique semitegens, presertim ad suturam obsoletissime, sub lente vix perspicne. spiraliter striatus; apertura diagonalis, ad apicem nsque pervia, transversim emarginato-elongato-ovata, " latior quam altior, marginibus callo tenuissimo junctis, supero subrecto, infero arcuato, columellari membranaceo-limbato, breviter reflexo-adnato, duplicationem formante. 
Diam. maj. 11/1/2, min. 8 ; alt. 7 ; apert. lat. 8 , alt. 5 mill.

Animal pustulatum, fuseo maculatum; pes longissinus. ad tergum valde compressus, margine fusco-marulato. subtus unicolor, tripartitus, partibus lateralibus transversin sulcatis.

Habitat in Camerunia: Bibundi, Vevoka, Itoki (S.).

Specimina 11, omnia viventia collecta, exstant.

La sculpture embryonnaire est chez cette espèce encore plus régulière et plus fortement accusée que chez la précédente (Voir Pl. II, Fig. 5).

Lianimal est entierement tacheté d'un gris foncti tirant sur le brun; même le bord du disque locomoteur est orné d'une série de taches de la même sorte que nous avons décrite chez Aspidelus Chaperi Mor.

Le pied est très prolongé en arrière de la coquille et comprimé des denx cotés. Il est convert d'une granulation serrée peu saillante, et en outre les lobes du manteau sont ornés çà et là de tubercules inégaux, assez larges. Le test est revêtu diun épiderme extrêmenent mince. se detarhant fort facilement; il est coloré d'un jaune paille pâle.

Helicarion depressus 11. sp.

Testa valde depressa, peripheria suhauriformi. tenuis. ir-

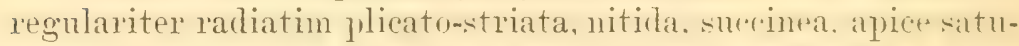
ratiori; spira planata apice sulperominulo: anti. 2012 suturit suluanaliculata, adnata discereti; embryonalis spiraliter punctato-striatus, ultimus relerrime creseens, planatus, arl peripheriam compressus, penultimi maximam partem obliyue tegens. sub lente undique ohsoletiscime of crelerrime spiraliter strial-

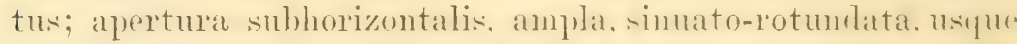

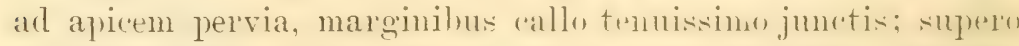

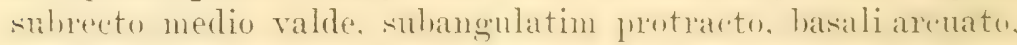

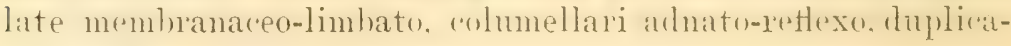
tionem fere formante.

Diam. maj. $13^{1} / 2$; min. $9^{1}, 2$; alt. 6 mill.; apert. diam. maj. $9^{1 / 2} ; \min .8^{1 / 2}$ mill. 
Animal granulosum, fusco maculatum; lobi pallii granulis requalibus, nec pustulis, ornati.

Long: 37 mill. Pes quasi costis parallelis, obliquis ornatus.

\section{Habitat in Camerunia: Bonge. (S.).}

Specimen unicum, in alcoh. servatum, in coll. mus. zool. Univers. Upsaliensis exstat.

Helicarion depressus est évidemment très roisin de $H$. columellaris m., mais la forme aplatie du test, la seulpture spirale beaucoup plus accusée, le développement accéléré du dernier tour l'en distinguent néanmoins. L'animal présente aussi quelques particularités. Les lobes du manteau recourrant une partie de la coquille proportionnellement plus petite, sont ornés d'une granulation fine, serrée, manquant tout à fait ile tubercules et ressemblant parfaitement à relle de $H$. pertenuis $\mathrm{m}$. Relativement à la coquille et au pied des autres espèces du genre ci-décrites, le pied est d'une grandeur' inorme. surtout la partie postérienre, qui est, en outre, obliquement ornée de côtes filiformes, parallèles, d'une nuance pâle. Mais le seul individu que nous ayons devant nous étant conservé dans l'alcool, il ne faut pent-être pas attacher trop d'importance ì ce dernier caractère, très singulier.

La coloration ressemble exactement à celle de H.columellaris.

\section{Helicarion subglobosus n. sp.}

(Pl. II, fig. 9-14.)

Testa ublique subglobosa, temuissima, irregulariter radiatim striata, nitidula, pallide albido-straminea; spira prominula; anfr. 3, sutura adnata, angustissime marginata discreti; embronalis sub lente vix perspicue spiraliter striolatus, ultimus regulariter sat modice crescens; apertura ad apicem usine perspicua, emarginato-rotundata, margine supero sensim arcuatim descendente, infero et columellari late membranaceo-limbatis, angulo obsoleto inter se junctis. う mill.

Diam. maj. $8^{1 / 2}, \min .6$, alt. $6^{1 / 2}$; apert. lat. $5^{1 / 2}$, alt 
Animal pallide rufescens, an tergum rompressum, fusconebulostm rel unirolor. finsco-brumenu; prosesi pallii tulnliforme pustulati. Per pallide marginatus. retioulatim sulcatus, vix pustulatus, subtus pallidus, micolor, tripartitus. partibus lateralibus transversim sulcatis.

Habitat in Camerunia: Itoki, Vevoka (S.).

Specimina 4, viv. collecta, in mus. reg. Holm. exstant.

Même à la loupe la plus forte, il n'est guère possible de reduire en point, les stries spirales à peinte perceptibles du tonr embrymaire, comme che\% les trois espees pridentes. Par sa forme, cette espèce ressemble beanconp a Titrina

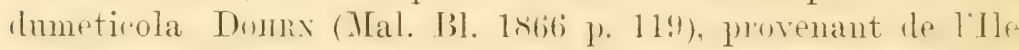
du Prince. La coquille est globuleuse, d'une couleur jaune blanchatre. Les bords de la columelle et de la hase sont largement membraneux.

Le bord intérieur des tours de la spire étant tout-à-fait libre, l'apex est visible de la base à un degré encore plus haut que chez les deux précédentes.

Par la coloration de l'animal, cette espèce se rapproche

plus de $H$. pertenuis, tandis que sa granulation est tres ressemblante it celle de $H$. columellaris. dont elle diffexte toutefois par les tuberules des lobes plus serres moins lareres. at d'me apparene prescue tubiforme. Pent-ritre cela répend-il cependant de la conservation dans l'alcool.

Il est fort douteux que les quatre espèces que nous venons de derrire aprartiement réellement an meme genre yue Helicalron plicatulus r. Martwis. Elles en difféent par la sulpture embrymaire par la forme des lobes du mantean it par la gramulation de la surface du corps.

\section{Gemus Zonitarion Pfefferr.}

\section{Zonitarion semimembranaceus (v. MARTExs).}

$$
\text { (Pl. I, fig. 44-48.) }
$$

1876 Helicarion semincmbranacens v. Mantens Monatsber. Berlin 1 . 253, 'T'. 1, lig. 1-4.

$188: 5$

'Truon Man, of Conch. Ser. 2: P. 3. p. 184, Pl. 43 , Fig. $52-54$. (Zonitarion) ThYon ibidem, appendix P. i. 1). 253. 
BIIANG TILL K. SV. VET.-AKAD. HANDI, BAND 22. AFD. IV. X:0 2. 35

Kantérom: Bomana (1100) m. d'alt., D.); Bonge. Itoki Na N'Golo, Bibundi (S.); (Victoria (B.)).

(Togoland: Bismarckburg (BüTTNER).)

\section{6 spécimens conservés dans l'alcool.}

En comparant les figures qu'a jointes M. V. MLARTENs à sa diagnose sur cette étrange espèce avec celles que nous en avons données, on ne pourra s'empêcher de constater des différences asse\% considérables. () res riches matérianx que nous avons à notre disposition nous laissent soupcommer que la coquille reproduite par ce savant était en état de dessication. N'ayant vu que des individus conservés dans l'alcool, nous ne jouroms pas juger des figures reprisentant l'animal à l'état vivant, mais elles nous paraissent néanmoins un peu extraordinaires. Chez les nôtres, on aperçoit, sur. la partie du mantean qui enveloppe la spire de la conuille. un pli externe en forme de carène an nivean de la face supérieure du test. Ce pli existe même chez les individus les plus jeunes. La seule chose qui ne s'accorde pas avec la description qu'a faite M. v. ML.nTws, sant naturellement en re pui concerne le hod inf'irieur le l'ouverture (dépendant, comme nous l'arous mentionné ci-dessus, de l'etat de la corpuille), c'est que nos spécimens sont gravés de stries spirales d'une finesse excessive, à peine perceptibles à la lompe, et que sur la plupart on roit en outre a et là des sillons interrompus irréguliers. probablement d'mue nature secondaire quil faut considérer plutôt comme des perturbations dans le développenent de la coquille. La coloration de l'animal est très variable. La plupart de nos individus sont ornés de taches noirâtres plus on moins tirant sur le brun et quelquefois tout à fait noires, mais il y en a aussi d'une nuance unicolore, pâle ou obscure.

La partie centrale du disque locomotem triparti est toujours pâle, et ordinairement les parties latérales sont de la même conleur, mais quelquefois, surtout rhez les spreimens o)sururs unicolores, elles sont de la même nuance que le reste du pied. A l'exception de la partie des lobes du mantean Iont est revêtue la face opposée membraneuse de la conuille, l'animal est entierement convert d'me gramulation fine et égale.

L'espèce vit surtont sur les fenilles de Pennisetum polystachyon SchulT. 


\section{Fam. ZONITID压.}

\section{Genus Thapsia Albers. \\ Thapsia troglodytes (MORELET).}
1848 Holix troglodytes Morelet in Revue zool. p. 351.
184! Africana Pfeiffer Proc. zool. Soc. of London, Nov. p. 128.
$1 \times 52$
1.:3: troglodytes PFeiffer Mon. Hel. III p. 77.
2
1854
PETT in Journ. de Conch. 'T. III p. 68. troglodites $>$
Fig. $14-16$. p. 67 , 1'1. 1 , Africana Reeve Conch, icon. Vol. VII, Pl. $197 \mathrm{~N}: 0$ 1385.
iroglorlytes
Pl. 198 N:o 1393
1858
1860 Nimina
Monerdes Sér. conch. p. 11, Pl. 1, Fig. 1.

1865 Helix Liberize Brown in Truon Amer. journ. Conch. 1 p. 136. 1866 troglodytes PFEIFFer in Chemn. ed, nov. III p. 419 , T. 148 , Fig. 15,16 .

Nanina $; \quad$ v. Martens Malak. Blätter p. 103.

1868 Helix Liheria Monelet Voy. Welw. p. 45.

1876 > PFEIFFEL Mon. Hel. VII p. 96.

Nanina troglodytes v. Martens Monatsber. Berlin p. 254, '1. 1, Fig. 9, $9 \mathrm{~b}, 9 \mathrm{c}$.

1881

1885 Xesta

Buchliolzi

1891 Nanina troglodytes
(Thapsia) Pfeirfeli Nomenclator p. 56.

Tryon Man. of Conch. Ser. 2: P. 6 p. 128 Pl. 42 , Fig. $18-20$. 'T'rYoN ibidem Fig. 90.

v. Martens Sitzungsber. d. Gesellsch. naturf. Freunde, Febr. p. 30.

v. Martens Mitheil. aus d. d. Schutzgeb. Band VI, Heft 3.

Kaméroun: Etome, Basse, Bibundi (D.); Bomana (J.), N'dian (S.); (Bonjongo (B.), Barombi (Pr.)).

(frind Bassam. Axim; 'Togoland: Bismarchburg (BütNER).

Gabon (WELWITSCH);)

45 spécimens, dont 40 recueillis a l'état virant. 
BIHANG TILL K. SV. VET.-AKAD. HANDL. BAND. 22. AFD. IV. N:0 2. 37

La plupart de nos spécimens sont d'une conleur rougeâtre, mais il y en a anssi quelques-uns qui sont presque blanes ou d'me teinte jaunâtre tirant sur le vert. Chez tous les sujets en bonne comlition que nous arons examinés. la suture est d'une nuance violette plus ou moins distincte, comme l'a décrite MI. MLoreusit; on peut done supposer, que les sujets que MII. Petit et Albers ont eus à leur disposition (trois seulement) ont étẻ recueillis vides et décolorés.

La surface supérieure de la coquille, les tours embryonnaires exceptés, qui sont prestque lisses, est gravee de stries décurrentes granulenses, perceptililes senlement it la loinpe; sur le côté opposé, ces stries deviennent encore plus fines et plus ondulées, et leur granulation s'émousse it mesure fu'elles: se rapprochent de l'ombilic. ('ette grannlation domme it la connille un éclat mat d'me apparence soyense on plutit grasse. Sous l'épiderme, membrane excessivement mince et se détachant très facilement, le test est tout-ì-fait sans éclat.

Le manteau de l'animal est orné de petites taches noires, irrégnlieres, perreptibles par transparence it trasers le test.

Les sujets provenant d'Etome sont d'une fragilité extrême.

Le plus grand de nos sujets mesure 28 mill. de diam. max. et 24 de diam. min. sur $14^{1 / 2}$ mill. de hautenr.

Nons avons entre les mains des individus qui representent des formes transitoires entre les types reproduits par MII.

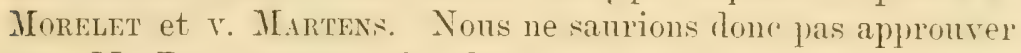
que M. Bovrguiganat ait élevé à la dignité d'une nouvelle espèce, sous le nom de Nanina Buchholzi, la connille que, le premier, M. BécunoLz a rapportée de Bonjongo it Kamérom, et que MI. v. Martens a représentée dans son onvrage.

Selon MI. v. Martess, l'angle du dernier tomr manquerait che\% cette espèce; or l'existence d'un tel angle nous sembltr nécessairement résulter de la description, terminée ainsi par l'éminent savant: Das Gewinde erhelst sich als breiter Kegel mit etwas concavem Profil aus der abgeflachten olseren sieite der letzten Windung, während deren Unterseite stark gewölbt ist; et, certes, on l'aperẹoit aussi sur la figure qu'il y a ajontée. Cet angle, plus ou moins fortement marqué et

1 Selon II. TRYor, on pourrait croire que cette localite se trouve i Mozambique. Elle est située sur la pente méridionale du petit pic de Kaméroun, au nord de la ville de Victoria. 
- effarant sumvent vers l'omerture, ni a pontant jamais l'ap-

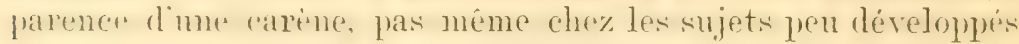
dont nous possédons un grand nombre. Lidentité de cette

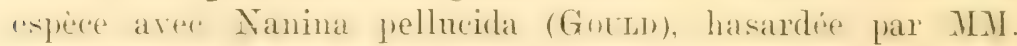
Pefeffer et Tryon, est donc inadmissible.

Nous fiant a MI. Morelex, nous avons cité Helix Liberice Browx parmi les synonymes de cette espèce, mais comme M. Pferfer la déclare encore plus carinée que $H$. pellueida GouLr, nous sommes disposés ¿̀ l'en faire disparaître.

\section{Thapsia calamechroa (JoNAS).}

$184:$

1848

1850 Nanina

1854 Helix

1855 Macrochlanys

1860 Nanin?

1866 Helix

Naninit

1876

1881

18 si) Xestia
Jonas (Olim in litt.).

calamechroa Jonas, Philippi Atbild. I: 3, p. 47 . Helix 'Tab. III, Fig. 2.

JFerferer Mon. Hel. I, p. 57.

(Xesta) Albens Heliceen p. 59.

Reeve Conch. icon. Vol. V'II Pl. 179.

N:o 1231.

Adaxis H. et A. Genera of rec. Moll. p. 224 .

(Thansia) AtBeis Heliceen ed. Y. MAKTENS P. 56.

Pfeiffer, Chemn. ed. Kï̈ster. III, p. 319, T. 129, Fig. 11, 12.

v. Mantens Malak. Bläiter p. 103.

Monatsber. Berlin p. 255 .

T. 1. Fiщ. 111, 11.

(Thapsia) Prenfer Nomenclator p. 56. 》Tryon Man. of Conch. Ser. 2: P. 6, p. 127, P'l. 42 , Fig. $8,9$.

Kaméroun: Mokundange, Batoki (D.); Bonge, Itoki Na $N^{N G}$ Golo, Kitta (S.); N'dian (D., S.); Bibundi (S., J.).

(Lagos; Côte d'Or: Aburi (B.).)

24 spée., tous recueillis à l'état vivant.

A mesure que la coquille se développe, la coloration devient plus pale, sans derenir pourtant anssi patle pue dans la itgure qu'en a domee .I. Jox.s. Les stries décurrentes, qui sont d'une tinesse excessive, ne sont pas granuleuses comme 
.he\% la mécédente et. en outre. elles ne changent pas d'apparence sur la face opposée du test. La suture devient par transparence un peu marginée.

Nous soupconnons que la figure de l'animal de cette espèce qu'a reproduite M. v. Martexs d'après le dessin de II. Buchnolz, n'est pas entièrement juste. Chez la plupart ie nos sujets, qui ont été recneillis en très bon état, l'extrémite dn pied de l'animal au-dessus du pore terminal, tantôt se termine en forme de bec, tantôt est prolongée ultérieurement en un processus ayant l'apparence d'une corne. Il parait que cette corne se détache fort aisément, et probablement 1I. Bronnor a fait son dessin d'apres $u$ individu qui l'avait déjà perdue. Il y a cependant lieu d'ajouter que ces obserrations sont faites sur des indiridus conservés dans l'alcool. La pied est, même chez les sujets conserves de la sorte, d'une longueur extraordinaire en arrière, et gravé de sillons obliques parallèles.

\section{Thapsia Sjöstedti n. sp.}

(Pl. II, Fig. 15--20.)

Testa vix perforata, globoso-depressa, tenuissima, pelluvila, sericeo-nitida, albido-vitrea, presertim subtus regulariter plicato-striata, sub lente lineis spiralibus. subtilissime granulatis, decussata; anfi. $5^{1} 2$, parum convexi, sensin accrescentes. olsoletissime rotundato-angulati. angulo in ultimo evanescente; sutura adnata, plicata, sulmarginata; apertura lata, lunaris: peristoma rectum, acutum, marginibus distantibus, callo temisino junctis, columellari reflexo, perforationem fere tegente.

Diam. maj. 19 ; min. 16 ; alt. 11 mill.

Animal reticulatim sulcatum; pes postice arl tergum nigrescens, truncatus, supra cauda longa instructus.

Habitat in Camerunia: inter Mundemha et Mattamani (ぶ.).

Un grand nombre de spéc, recueillis à l'état vivant.

Évidemment rette nouvelle espèce est très voisine de la pricédente, mais les particularités qu'elle présente l'en distinguent fort aisément.

Le test est un peu plus convexe, et l'accroissement des tours de la spire est un peu accéléré. On voit, en outre, sur 
les tours les traces d'un angle qui s'émousse à mesure que la coquille se développe. L'ombilic est à peu près couvert jar le bord "olumellaire. réthehi a un phus hant degri ym. chez Th. calamechroa. Les stries spirales, perceptibles seulement à la loupe, sont très finement granulées, et la

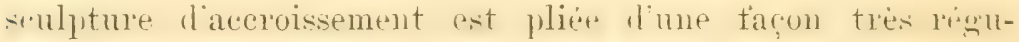
lière, surtont à la face opposée.

La coquille est extrêmement diaphane, at pen pres hralint. ot beamoup plus mince que Thapsia "alamechroa. ('hr\% la coquille vide, la couleur devient presque laitense.

La suture est plus distinctement marginée et pliée d'me İaçon plus régulière; elle monte, en outre, plus haut sur le tour précédent.

Le pied de l'animal n'est, chez cette espèce, ni aussi prolongé ni aussi comprimé aux côtés que chez la précédente. Il en résulte qu'il est moins haut, surtout ver's son extrémité, et que celle-ci devient plus arrondie; d'aillems le pied parait plutit réticule, les sillons ohlinges neitant par si prédominants. Le bout du pied se prolonge en un processus beancoup plus long, n'ayant pas la forme de bec. Ce n'est que très rarement que cet appendice manque. La coloration de lanimal est anssi dittérente che\% ces denx espiras. Chez la nôtre, ce n'est que vers son extrémité que le pied devient un peu grisâtre, la nuque de l'animal, ainsi que les lobes du manteau, n'étant done pas d'une nuance noirâtre. Au lieu de la bande noire bordée d'un blanc tirant sur la "rime que montre par transparence le manteau de Tho. s.jistedti, "alui de Th. alamechroa presente denx siries spirales de petites taches d'un gris brum, irrégulières et beancoup moins maryures. Tous nos sujets se ressemblent exactement.

Cette espèce semble beaucoup plus rare que l'autre, n'ayant été rencontrée que dans une seule localité. M. Sıöstedt, qui l'a découverte, et anquel nous la dédions, nous a conrmuniqué ce qui suit sur sa fréquence: „Partant de N'dian wt mavancant pendant les premiers jours du mois do juin 1K91. answitot apres le commenement de la saivon des pluies. vers l'intérieur du pays, je rencontrai cette espèce en crande abondance, surtont intre Mundemba et Mattamani. alupes du semtier pui traverse la hrousse épaisse et humide. (Buschwald). File sy trouvait sur le sol parmi les femilles pourries, et çà et lì elle était si fréquente, que j'ai pu, sur 
BIHANG TILL K. SV. VET,-AKAD. HANDL. BAND 22. AFD. IV. N:0 2. 41

un seul point, en ramasser ume poignée. Saut' d cette occasion, je ne l'ai jamais rencontrée.

\section{Genus Trochozonites Pfeffer.}

\section{Trochozonites Ibuensis (PFEIFFER).}

1846 Helix

1848

1854

1876 'T'rochonanina

1881 'L'rochomorpha

1885 Rhysota
Ibuensis Pfeiffer Symbola III p. 66.

Mon. Hel. I, p. 51.

ReEve Conch. icon. Vol. VII, PJ. 199, N:o 1399.

v. Martens Monatsber. Berlin p. 256 , T. 1 , Fig. 15.

(Nigritella) Pfeiffer Nomenclator p. 80. (Trochozonites) TryoN Man. of Conch. Ser. 2: P. 5, p. 51. Pl. 24, Fig. 95, 96

Trochozonites Ibuensis var. tumidulus ( $\mathrm{v}$. MARTENS).

1876 Trochonaniua tumidula v. MaR'Tens I. c. p. 256, T. 1, Fig. $12-14$.

1885 Rhysota tumidula (Trochozonites) Tryon 1. c., Fig. 97.

La forme typique: Kamérom: Itoki Na N'Golo, N'dian. Kitta (S.), Isowi, Bonge (D., S.), Bibundi (D., S., J.); (Bonjongo (B.)).

(Ibu ou Ibo près du fleuve Niger).

La variété: Kaméroun: Batoki (D.), Kitta (S.), Boana. Buea (à 1000 m. d'alt.) (J.), Bibundi (D., S., J.); (Bonjongo (B.)).

Nous possédons des deux formes un grand nombre d'individus, dont la plupart sont conservés dans l'alcool.

Nous avous considéré Trr. tumidulus (v. Martens) comme une variété de Tr. Ihuensis (Pfenfer), l'examen attentif d'un grand nombre d'exemplaires des deux formes ne nous permettant pas de le regarder comme une véritable espece.

Les spécimens, ront la coquille est plus mince et plus aplatie et par conséquent la carene plus tranchante, la couleur d'une nuance cornée, plus ou moins pâle, la carène de la même couleur que le reste du test, et enfin dont la 
soluluture dans les deux sems est moins fortement maryue. applatiennent, selon nons. a l'esperee de M. Prestrak, tandis que nous considérons comme une forme variante, plus déreloppere sous tom les rapports. ceux dont la copuill. est plus grande et plus rentrue, les tours de spire plus convexes, la sculpture plus reguliere ot plus fortement prononcée. la conleur d'un brun foncé, xougeâtre, sur lequel la carène obtuse se présente comme une bande pâle. C'e sont le 'T'r. tumidulus de M. v. Martexs.

Entre ces deux formes il y en a de transitoires, surtout en ce qui regarde la taille.

La sculpture spirale, qui doit bien être le caractère spé-

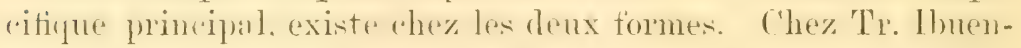
sis, elle est, surtout quant aux coquilles vides, d'une extrême faiblesse, n'étant perceptible qu'à une loupe assez forte, ce qui a porté 1L. Pfeiffer à la négliger.

Chez Tr. tumidulus, cette sculpture, quoique très fine, ut tomtefois asser visible sur les derniers tours, principalsment près de la suture, et avant tout sur les tours supérieurs, où elle produit de petits noends sur les côtes transversales; elle est, en outre, toujours moins fortement accusée dans les intervalles de la sculpture transversale.

Chez la forme principale, le manteau de l'animal est semé de petites tacher noires et blandes (les dernieres manyment quelquetois) quom apereoit gar transparence. tandis que celui de la variete est prespue entierement teinte de noir. tarleti seulement cà et là d'une nuance plus pâle tirant sur le gris et invisible du dehors. Chez tontes les deux, l'extrémité du dos du pied et la petite corne y attachée sont d'une "oulen moratre plus foncée ef plus distinctement limition dans la variété.

Cette espèce, comme toutes les autres appartenant au srompe des Trochozonites, prisente un sculpture embryonnaire singuliere à latpuelle nous attarhons heancoup d'importance comme caractere générique. Elle se rompose, al l'exchsion de toute antre smulpture, de tines stries tiliformes, decurrentes, d'me nature tont it tinit dittirente de celle de l'autre sculyture spirale. Ia face opposée est grave de stries excessive ment fines. legerement onduleuses. a lapparence sensiblement gramulense par suite des stries rayomant du centre de la base. On rencontre cette senlpture basale, modifiée pent- 
itre dans l'un ou l'antre sens, che\% tons les Trochozonitess et quelque chose danalogue se retrouve chez le genre Thapsia.

Lans plusiens individus, appartenant aux deux formes ot recueillis pendant les mois d'avril- juin, nous avons troure de nombreux embryons.

La forme typique vit avec sa variété et plusieurs de ses rongéneres principalement sur les feuilles tres aromatipues

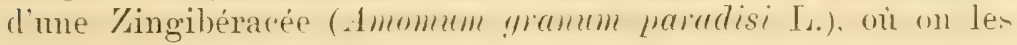
trouve d'ordinaire très fréquemment.

\section{Trochozonites reticulatus n. sp.}

(Pl. II, Fig. $26-31$.)

Testa semiobtecte perforata trochiformis, tenuis, diatphana. straminea vel pallide rornea, sericeo-nitidnla; spira mina. apice obtuso; anfr. nunc convexiusculi, nume subplanati; emhryonales suh lente distincte spiraliter filo-striati, sequentes proximi dense, oblique plicato-striati et, sculptura embryonali contiunata, pulcherrime reticulati; ceteri liris tenuissimis. distantibus decussati, liris siepe in anfr. duobus ultimis ant evanescentibus ant tantum prope carinam perspicuis; ultimuplanatus et siepius sulb-horizontaliter deviuns, angulum cum penultimo formans, acute et compresse carinatus, hasi sat convexus, ad carinam leviter excavatus, radiatim levisime striatus et undique striis spiralibus undatis, sub lente vix perspicuis, interdum hic illi, filiformibus, ormatus, sericesnitens. Sutura impressa, in ultimo subcanaliculata et interdum carina marginata. Apertura obliqua, sulsecuriformis. Peristoma simplex, rectum, tenne, marginibus callo tennissimo junctis; columellari arcuato, in hasalem sensim abeunte. breviter reflexo, perforationem semitegente.

Diam. maj. 13-16 mill.; min. 11-13; alt. 9-11 mill.

Animal pallidum, pallio maculis nigrescentibus et albis. translucentibus ornato; pes postice vix truncatus, cauda longa, nigra, ab extremitate sat remota, instructus.

Habitat in Camerunia: Bonge, N'dian, Kitta, Itoki Na $\mathrm{N}^{\prime}$ Grolo (S.). 
Specimina 35 exstant, omnia viv. coll.

Il n'y a que peu à ajouter à la diagnose que nous venons le domner de cette nouvelle et belle espèce.

Elle s'approche sans doute de la forme typique de Tr. Ihuensis, dont alle differe toutefois, entre autres, par lat forme deprime de la rounille, par les tomes plus aplatis par lit wulpture, par la coulem jaune paille et enfin par la suture. yni. entre les deux dernicrs tomis de spire, est canaliculie pt. quelquefois marginée par la carène, fort tranchante. Les tarhes blandhes du mantean de l'animal sout prodominantes ot quelquefois très grandes.

\section{Trochozonites Lindströmi 11. sp.}

(P1. II. Fig. 40-42.)

Testa semiohterte pertorata,subgloboso-conica. solidinsenla pallide cornea vel albida, subtus pallidior, sublactea, subpellucens. serieen-nitidula; spira conica, apice subprominulo; anfr. 7 , convexi, tumidi; primi spiraliter, dense, oculo nudo haul conspicue, filo-striati; seyuentes oblinue, densissime irregulariterque plicato-striati, sub lente striis olsoletissimis decussati; ultimus subinflatus, merlo pallide cingulatus, olsolete filo-angulatus, basi convexiusculus, sub lente undique trunissime, spiraliter. undatim striatus, sulcis paucis. nneentriris sirpissime soupptus. Sutura filomaromata, inpressa. Apertura diagenalis, subsemicirenlaris; peristoma rectum. arutum. regulariter arenatum. marginilus "allo tenuissimo junctis, columellari triangulatim reftexo, allidlo-incrassato.

Diam. maj. 15, min. 13; alt. 13 mill.

Animal pallidum, minolor; pes postices randa nigriscente. mediocri instruetus.

Halitat in ('amernuia: Mnkmulange, Etome, Kitta, Basse. Batoki (D.)

Specimina multa, omnia riventia collecta.

Cette espèce se rapproche beancoup par sa forme de Tr. Ihmpnsis val: tmmidulus (V. MAnTws), mais alle presente des 
particularités qui l'en font très facilement distinguer. La coquille est plus solide, d'un éclat mat soyenx et d'une couleur cornée très pâle, ou, surtout à la face opposée, d'un blanc tirant sur la cire.

La carène est encore plus obtuse, et ehez les individus yui ont atteint leur taille détinitive, elle s'effaces presifue entierement an dernier tour, on y présente l'apparence d'un til aplati. Sur les coquilles d'me nuance cornée, elle se dessine comme une bande pâle et toujours elle est plus diaphane que le reste $d u$ test.

La sculpture de l'accroissement est beancoup moins fortement arcusée, se composant de stries faibles, pliciformes. et jamais de costules. La surface est en outre gravée de stries derurrentes à peine perceptibles a une forte lompe of s'imoussant sur le dernier tour. La sculpture embryonnaire et celle de la base est la même que chez les autres espèces du gemre, mais à la base on apereoit encore le plus souvent chez les individus adultes une zone gravée de sillons concentriques marqués, mais peu profonds, facilement risibles à l'oeil nu.

L'animal est unicolore, pâle, manquant entièrement de taches, et le processus seul de l'extrémité du pied présente une nuance noirâtre.

M. Dusḱn l'a trouvée en grande abondance à l'époque des secheresses les plus intenses sur les fenilles de Theobroma racao $\mathrm{L}_{\text {., }}$ ayant l'ouverture termée d'une membrane tris mince.

Nous dédions cette belle espèce à M. le Professeur G. LindströM.

\section{Trochozonites suturalis $n$. sp.}

(Pl. II, Fig. 43-46.)

Testa subobtecte perforata, subglobosu-conoidea, tenuis. superne oblique et regulariter "ostata, "ornea, rix nitidula. subdiaphana; spira conica, apice acutinsculo; anfi. 6 - 7 convexi, spnsim accrescentes, subgradati, sursum ad suturam horizontalem, nitidam angulati; embryonales striis spiralibus. tiliformibus ornati; ceteri costati, in interstitiis sub lente obsoletissime, spiraliter striolati; ultimus infra merlinm filurinctus, basi mediocriter convexus, radiatim striolatus, nitidulus, sub lente tenuissime densissimeque. undulatim, spiraliter 
striatus; apertura oblipua, subseruriformis; peristoma simplex. peotum. marginibus wallo tenuissimo junctis, rolumellari sulallosn. superne lueviter reflexo, jerforationem fere tegentr.

Diam. maj. 7 mill.; min. $6^{1} / 2$ mill.; alt. 7 mill.

Animal minolor. aut nigresens ant pallisum; per pustion rix truncatus, supra cauda brevi, conica instructus.

Habitat in Camermia: N'dian (S.).

Specimina 5 exstant, "quorum :3 viv. coll.

Les côtes très marquées dont est ornée la surface, sont espacées entre elles par des intervalles assez larges et très réguliers; en traversant la suture, ces côtes s'affaiblissent sensiblement. La suture présente la particularité la plus "arartiristique: elle est légirment billante, les stries spirales fines dont est gravé le reste du tour y manquant; elle est en ontre presque horizontale, ce qui donne a la spire une apparence étagée. Les toms embryomnaires et la base ont la seulpture ordinaire.

\section{Trochozonites Adansoniæ (MoRELET).}

1848 Helix Adansonia Monelet in Revue zool. p. 351.

$1853>$ I'FEIFFER Mon. Hel. III p. 59.

1858

Morelet Séries conch. I p. 13, 1'l. I, Fig. 4.

1881 Hyalinia

1885 Iihysota

(Counlus) Pfejfaer Nomenclator p. 74 .

1894 (?) Ninina

('Trochozonites) 'l'ryon Man. of Conch. Ser. 2:

P. 5, p. 52, Pl. 24, Fig. 3. Girari in Jornal de sciencias, Lisboa, 'I'. ") N:o XI p. 204.

Kamérom: N'dian (S.).

(Ile d'Anno-Bom (?); Gabon.)

Un seul spécimen.

Une particularité qui ne se rencontre chez ancune antre

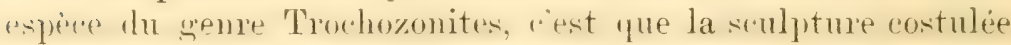
du reste de la coquille se présente même sur les tours emtrromains. pre qui rend la sonlpture spirale gimerique, en

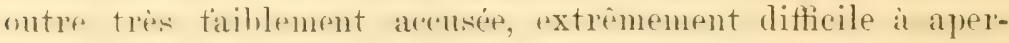
cevoir même au microscope. 
La sculpture concentrique de la base est de même entièrement imperceptible à l'oeil nu.

Il semble que cette espèce, peu commune selon M. MoRELET à Gabon, l'est également à Kaméroun, un seul individu y étant trouvé.

\section{Trochozonites Folini (MoreleT).}

1848 Helix Folini Morece' in Revue zool. 352.

1853 PaEITFer Mon. Hel. III p. 57 (excl. syn. Goild).

1858

MIORELET Séries conch. p. 13, Pl. 1, Fig. 3.

1866 Nanina

DOHRN MIal. Blätter XIII p. 120.

1868 Helix $>$ Morelet Voy. Welw. p. 56.

1881 Trochomorpha Folini (Nigritella) Pfeiffer Nomenclator 1\%. 80. 1885) Rhysota (Trochozonites) Tron Man. of Conch.

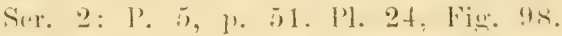

Kaméroun: Bibundi (D.); N'dian (S.); Bonge (D., S.). (Tllha do Principe (Folrv); Angola: Pungo Andongo (WELw.); Gabon.)

40 spécimens, dont 34 conservés dans l'alcool.

La diagnose domnée de rette espère par Mr. Moreletr saccorde exactement avec nos individus; ils sont cependant plutôt colorés d'un rouge brun que d'une teinte cornée, la nuance rouge n'étant toutefois pas aussi prononcée yne sur la figure qu'en a foumie M. Morelet.

Le côté inférieur de la coquille est d'un brillant très vif, et à peine aperçoit-on sous le microscope des traces de la sculpture spirale qui caractérise ce groupe. Chez les sujets adultes, le bord inférieur de l'onverture prés de la rolumelle sst légèrement arqué en avant. La circonférence de la spire est quelque peu convexe, vers le sommet seulement médiocrement concave.

Il est assez étonnant que M. Pfeiffer (Mon. Hel. 1. c.) ait pu réunir Tr. talcosus (Gocti) avec cette espèce, lle même yne M. Gorto semble disposé à le réunir avec Tr. Ibuensis (Premffer). Plus tard (Mon. Hel. VII p. 101), M. Premfere a rhangé sa manière de voir, informé de son erreur par MIM. Morelet et DoHrN (1l. ce.).

M. Dohrn (et après lui M. Pfeiffer) est le senl qui décrit la suture comme canaliculée; cependant ce caractère, 
tres distinct rhez tums nos spereimens, est d'me rertaine importance pour definir la pelation entre 'Tr. Folini (Mros.) et l'espèce suivante.

Trochozonites Folini var. percarinatus (v. MIRTFNs).

1876 'Trochonanina percarinata V. MARTEns Nonatsber. Berlin p. 256, 'I. 1 , Fig. $16-18$.

1878

G. PFEFFER Archiv für Naturgeschichte Band 44 p. 256 . T. 13, Fig. 5, 6.

1885 Thiysota ('Trochozonites) Trron Man. of Conch. Ser. 2: P. 5, p. 51, Pl. 24, Fig. $92-94$.

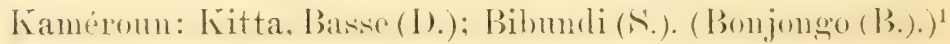

46 spécimens, dont 3̈8 recueillis ì l'état vivant.

Il est assez douteux que cette espèce puisse être recommue comme réellement distincte de Tr. Folini (Mor.), dont nous avons entre les mains des sujets parfaitement typioues. Les sujets sur lesquels se fonde la diagnose domnée par M. r. MARTExs et que nous arons eu l'occasion de voir au Musée de Berlin, ont perdu par l'usure mu "aractipe escentipl, savoir les petits processus pilitormes des cotes transrersales. rangus en séries spirales sétiformes, dont les restes ont donné naissance a cette observation de M. V. MARtexs: plimeis subtitissimis spiralibus decussatu». Or, ce caractère, ne s'y trourant plus. a tait disparatere ainsi la seule particularite qui distinguerait ssentiellement rette esperes de 'Tr. Folini (Mor.), et avec les côtes sétifères se présente au contraire un des caractères les plus distinctifs de ce dernier type. Il est impossible de déconsuir un autre carartire positivenent specifique indiscutable. Le seul auquel on put attacher de l'inportance, est celui que 'Tr. percarinatus est plus déprimé, d'où il résulte que la carène devient plus saillante, la suture plus distinctement ranaliculéx, ot la lase de la ropuille plus large.

Maris, outre des spreimens partaitement typinues des denx espèces, nous en arous des formes transitoires qui effacent

1 Le catalogue de 11 . Staungaer donne sous ee nom une espéce prorenant de Mozambique, nais cest sans doute une erreur. 
graduellement la différence, et dont la place dans l'me on l'antre espece est impossible it préciser. Par ces notif's, nous nous permettons de considérer l'espèce de M. v. Martens comme me rariété de Tr. Folini, et nous proposons pour elle le nom de Tr. Folini var. percarinatus (v. Martens).

La couleur de la coquille est la même chez les deux formes, peut-être un peu moins foncée chez la variété, et, autant qu'il est possible d'en juger par des individus conservés dans l'alcool, - les animaux sont également de la même nuance. La face opposée est en outre, par son éclat et par sa sculpture, exactement semblable à celle de la forme principale. Ce que dit II. Domrx de la strie saillante qui borle la suture de Tr. Folini, est également applicable à la variété.

Au surplus, elles vivent en compagnie sur les mêmes plantes, surtont sur les feuilles d'Amomum granum paradisi L., comme toutes leurs congénères.

\section{Trochozonites hystrix $n$. sp.}

(Pl. II, Fig. 32-35.)

Testa perforata, turbinata, pallide straminea, tenuis, diaphana, lamellis obliquis, transversalibus eleganter costulata, pilis longis, in seriebus spiralibus dispositis, in medio anfractum maximis, rigida; spira conica apice obtuso; anfr. 8, planati, sensim crescentes; embryonales spiraliter, dense striati, sequentes subplanati. lamelli-costati, piliferi; ultimus acute carinatus, basi sat convexus, nitidus, striis subtilissimis, oculo nudo haud conspicuis, sculptus; sutura carinato-marginata; apertura obliqua, securiformis; peristoma rectum, acutum, marginibus callo tenuissimo junctis; dextro subplanato, basali arcuato, colmellari breviter reflexo, perforationem fere tegente.

Diam. maj. 11/2, min. 10; alt. 11 mill.

Animal pallidum, maculis nigris et allidis, translucentibus adspersum; pes micolor, pallidus, postice canda nigra instructus.

Habitat in Camerunia: Kitta, Itoki (S.).

Specimina 9 exstant, omnia viv. coll. 
Les longs poils sétacés qui, presque sur le milien des trois dorniers toms. forment la troisieme sirie a partir de la carène, sont dirigés en hant et légèrement arqués. A l'insertion sur les côtes transversales, ils sont très larges et creux le long de leur côté gauche.

La face opposée est d'un brillant très vif. L'espèce appartient ividemment an mine grompe que Tr. Folini, mais la spire n'est pas concave vers le sommet, et la suture, qui est, rertainement, bordée par la "arene. 11 ist pas "analiculée. Ta couleur de la coquille est également différente.

Sur un fond noir, le manteau présente par transparence de grandes taches blanches à bord distinct, qui donnent à l'espèce une très belle apparence.

Le pied est unicolore, pâle, sauf à la partie postérieure du dos avec la petite corne y attachée, qui est d'un noir foncé.

\section{Trochozonites pilosus n. sp.}

(Pl. II, Fig. 36-39.)

T'esta jerforata. turbinata, pallide ornea, tenuis, diaphana. lamellis ohliquis, transersalibns, temuissimis, pilis brevibus, in serienus spiralibus dispositis, instructis. ornata; spira conica apice obtusinseulo, spiraliter, dense striato. Anfr. 8, convexi, sensim accrescentes; ultimus basi convexus, nitidus striis spiralibus, sub lente sat distinctis. sulptus, carina acuta, tenui cinctus; sutura carinato-marginata. Apertura ohliqua, securitormis; peristoma restum, acutum, marginilus callo tenuisimo junctis; dextero sensim arenatu, hasali profunde immerso, columellari subertienali, berviter, triangulatim retlexo, perforationem semitegente.

Diam. maj. 12, min. 101/2; alt. 11 mill.

Animal pallidum, nigreseente-nebulosum, maculis albis, translucentibus adspersum. Prs unicolor, postice cauda finsea, pro ratione magna, instructus.

Habitat in Camerumia: Kitta, Nilian. Itoki (s.); Moliundange (D.).

Specimina 17, omnia viv. collecta, exstant. 
Cette espèce, qui se rapproche beaucoup de la précédente, présente cependant quelques particularités distinctives.

La spire étant légèrement concave vers le sommet, et les tours un peu plus convexes, la coquille est de taille moins fortement conique. La carène est quelque peu plus tranchante, et les poils, à peu près égaux, sont rangés en séries plus nombreuses; sur le dernier tour, on en compte 9 à 10 , tandis que l'on n'en compte que $7 \mathrm{chez}$ la précédente. Tr. Folini a des séries encore plus nombreuses.

La base, du même éclat, off're à la loupe une sculpture spirale beaucoup plus distincte que chez Tr. hystrix. La suture est bordée par la carène, qu'on pent suivre jusqu'au commencement des tours embryonnaires.

La coloration de l'animal ressemble à celle de l'espèce précéclente, mais les taches blanches dn mantean sont moins larges, et la corne dn pied est d'un noirâtre moins foncé.

De Trochozonites Folini cette espèce diffère entre autres par la couleur de la coquille et par la suture non canaliculée.

\section{Trochozonites turbinatus $\mathrm{n}$. sp.}

(P1. II, Fig. 47, 48.)

Testa turbinata, ang'uste perforata, taleosa, vix nitidula; anfr. \& (?), convexiuseuli, irregulariter, oblique striati, ultimus obtuse, angulation filo-rarinatus; hasi radiation sat distincte sigmoideo-striatus; sutura filo-marginata. Apertura subtetragono-securiformis, diagonalis; peristoma acutum, rectum, marginibus callo tenuiswimo junctis, columellari subverticali. calloso-incrassato, breviter reflexo.

Diam. maj. 3, min. $2^{1 / 2}$; ait. 4 (?) mill.

Habitat in Camerunia: Kitta (S.).

Specimen unicum, apice destructo, in coll. mus, reg. Holm. exstat.

Le sommet de la coquille étant détruit. il est impossible de déterminer la sculpture embryonnaire. Celle de la face opposée est la même que chez les autres espèces du genre; les stries spirales en sont légìrement onduleuses, et celles de l'accroissement sont sigmoïles et assez fortement accusées. 
Trochozonites bifilaris (H. DoHRN).

(I'l. II, Fig. 49--55.)

1878 Trochonanina bifilaris H. DOHIn Jahrb. Malak. Gesellsch. p. 155.

T'esta perforata, conica, temuis, cornea, unicolor vel ticniis 1-3 eastaneis plerumque ornata, subdiaphana, leviter striata, sub epidermide tenuissima, submargaritacea vel sericea, tacile deterenda, sub lente subilissine decussata: sliua perfecte conica, apire acutinsulo. sub lente minutissine spiraliter striato; anfi. T-9 convexi, sensim arerescentes, sllprei medio carina ma, nltimus carinis duabus filiformibus, yuarum infera peripluerioa, cincti, sutura carinato-marginata discreti; ultimus hasi sat convexus, nitidisimus, radiatim striatus et striis spiralihns. suh lentr vix perspienis, sculptus, ad carinam obsolete excavatns. Apertura parum obliyna, rotumlato-securiformis. Peristona rectum, simplex, mareninibus distantibus, callo tenuissimo junctis, basali regrilariter arruato, columellari reftexo. perforationem semitegente.

Diam. maj. $5^{1 / 2-6} 6^{1 / 2}$ min. $5-6$; alt. $6-7$ mill.

Animal pallidum, postice nigrescens; pes auda nigra, pro ratione magna. instructus. postire rotundatus ner trumeatus.

Habitat in ('amerunia: Ekmmba-Liongo, Basce. Batoki (D.); Kitta, Bonge, Itoki (S.); Bibundi (D., S.).

\section{(Liberia (ScrweITzer).)}

Specimina is examinavimus, ommia riventia collecta.

La forme de cette belle petite espèce est celle d'un cône plus ou moins aigu, et le bord inférieur de l'ouverture est plus ou moins abaissé. Le nombre de fascies vaxie d'une à trois; en général il y en a deux, l'une, un peu plus large, se tronvant an milien des deux "arénes sur lat moitié inférienre des tomrs. lautles. Hus étroite. se rorant immediatement andessus de la "areme superieure. Dordinaire elles satfaiblissent it mesure que la conpille se dereloppe. et somrent elles s'énuniswent sur le dernier tour. surtout la superienue. La 
troisième fascie, très rare, se trouve à la base de la coquille près de la carene. Les fasuies et les carènes manquent toutes sur les tours embryonnaires. Les individus les plus rares sont ceux ornés de trois fascies et ceux qui n'en ont aucune.

Très rarement on trouve des individus qui ne possédent qu'une carène, celle de la périphérie, la supérieure ayant tout a fait disparu, on marynant quelyuefois sa place par une fascie plus trausparente et d'une nuance légierement plus foncée que le fond. Sur 78 spécimens, nous n'en avons tronvé que 3 de cette variété.

Il est hors de doute que l'espèce décrite ci-dessus est identiqne à celle un'a déconverte M. Sonwertzer à Liberia et qu'a décrite M. Dours (l. c.), mais le seul individu sur lenuel se fonde sa diagnose s'étant brisé ynand on l'a sorti de l'alcool, celle-ci est restée incomplète.

Trochozonites bitilaris est le plus voisin de Th. talcosus ((Goubd), et se distingue d'avec lni par nn épilerme singulier d'ime apparence talquense. L'apex et la base de la conpille sont gravés de la sculpture qui caractérise le groupe, colle de la hase étant tortefois à peine perceptible même à unt forte loupe.

Liaplendice du pied est d'me grandem extraordinaire relativement à celle de l'animal même.

On trouve le plus souvent cette espèce sur les feuilles d'une zingibéracée (Amomum granum paradisi L.).

\section{Trochozonites talcosus (A. GoULD).}

1850 Helix talcosa Gould Proc. Bost. Soc. III p. 194.

1853 》 Folini Pfeiffer Mon. Hel. III p. 57 adnot.

1869 talcosa $\gg$ Novitates Conch. III p. 500 T. 108 . Fig. $7-9$.

1876 》 》 Mon. Hel. VII p. 101.

Trochonanina taleosa var. elatior v. Martens Monatsber. Berlin p. 257.

1881 Trochomorpha " Pfeiffer Nomenclator p. 80.

1885 Rhysota $\#$ (Trochozonites) Tryon Manual of Conch. Ser. 2: P. 5. p. 52. Pl. 24, Fig. 2.

1893 Trochozonites talcosus v. Martens Mittheil. aus d. d. Schutzgebiet. Band II, Heft. 3. 


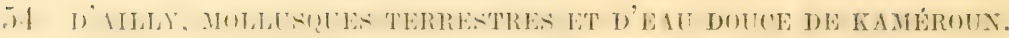

Kaméroun: Batoki (D.), N'dian (S.), (Bonjongo (B.), Buea (Pr.)).

(Liberia: Cape Palmas (Perkins));

('l'ogoland: Bismarckburg (BütTNER)).

2 spécimens recreillis à l'état vivant.

Par la sulpture, l'apparence talqueuse du test et la forme de la spire, cette espèce se rapproche beancoup de 'Tr. bifilaris (Domrs), par la seulpture senle aussi de Tr. Calabaricus (PFetffer). Elle n'a, au contraire, rien de commun aver 'l'r. L'olini, saut la soulpture singulière destours embrymmaires, qui rumeterise touten les espèes de cu genre, et il est ditficilo de comprendre comment M. PFisferer a pu la regarder comme synonyme de cette espèce-ci.

Les stries spirales très minces, microscopiques de la base, moins ondulées que d'ordinaire, ne sont pas granuleuses comme chez l'espèce suivante.

Mesurant 4 mill. et demi de diam. max. et 4 mill. de diam. min. sur 4 mill. de hauteur, et la spire comptant le nomble de tours. 6 et demi, que Ini assigne M. Pretrati, les specimens que nous aroms sous les yeux, comparis ì la variéte clutior (r. Martens l. ('). contirment la grande variabilite des dimensions que présentent les mollusques de ces contrées.

\section{Trochozonites Calabaricus (Preiffer).}

1856 Helix Calabarica Preiffer Proc. zool. Soc. London p. 327.

1859

Mon. Hel. IV p. 37.

1869

Novitates Conch. III p. 501. 'T'. 108 Fig. $10-12$.

1876 Trochonanina Calabarica v. Martens Monatsber. Berlin p. 257. 1881

1885 Rhysota PFeiffer Nomenclator p. 57.

('Trochozonites) 'TrYoN Man. of Conch. Ser. 2: P. 5. p. 51. Pl. 24, Fig. 99--1.

Kamirom: Kitta (I......). Nidian. Bonge (S); (Bomjongo(B.)). (Old Calabar.)

25 spécimens, dont 16 recueillis à l'état vivant.

Les petites dimensions, le nombre des tours de spire et les trumes margine colmellari superne vix reflexo démontrent que l'individu sur lequel M. Pferffer a fondé sa diagroses netait jois adulte. connme l'a sompenme ansisi l'illustre 
savant. J ̈undividu dont il a indique les mesures dans la Mon. Hel. VII p. 104, n'a cependant pas non plus atteint son complet développenent. car nons en avons des spécimens, uni, comptant un tour de plus, mesurent 17 mill. de diam. max. sur 10 de hauteur, et le bord columellaire chez les sujets adultes est tonjours superne hreviter reflexo, perforationem semitegrente. Le bord inférieur de l'muerture, près de la perforation, présente me faille sinmosité et décrit ensuite une légìre comrbure en arant.

La sculpture des tours embryonnaires est dı même type que chez les autres espèces de ce groupe; celle de la base, an contraire. est formée de petits grams perceptibles senlement au microscope, plus au moins disposés en spirale, remplarant les stries minces onduleuses de la plupart des Trochozonites.

Parfois la suture est marginée par la carène, lorsque l'enroulement des tours de spire ne coüncide pas exactement avec elle. La distance entre les stries filiformes décurrentes qui orment la surface supérieure de la coquille à partir du troisième tour, diminue vers la suture. L'animal est d'une couleur pâle, vers l'extrémité du dos d'une teinte grise noirâtre, et à travers le test on voit le manteau tacheté de noir.

\section{Trochozonites Théeli n. sp.}

(Pl. II, Fig. 21-25.)

Testa semiobtecte perforata, concavo-conica, tenuis, subopaca, rufo-cornea, apicem versus subtuspue pallidior. Spira concaviter producta, apice acutiusculo. Anfr. 10; embryonales sul, lente tenuissime, sulgranulatim, spiraliter striati, sequentes 4 lente accrescentes, convexinsculi, lamellis distantibus, obliquis, oculo mulo vix perspicuis. tuberculis obtuse instructis, costati; ceteri planati, plicis obliquis, arcuatis, in ultimo regularibus, et liris spiralibus, crebris temissimisque ornati; ultimus acute ef compresse carinatus, basi molice convexus, ad carinam excavatus, rarliatim plicato-striatus et (orulo nudo) sericeo-nitidulus, sub lente eleganter graunlis crebris, spiraliter dispositis. ornatus. Carina subuallosa, albescens, leviter undulata et prosertim subtus scrobienlo-marginata, suturam, primum impressam, deinde linearem, filomarginans. Apertura 
ohliyua, subtrapezialis. Peristoma simplex, rectum, marginibus callo teunissimo junctis. supero recto. basali leviter ar(uato. columellari subrerticali. cum hasali angulum tormante. breviter reflexo, perforationem semitegente.

Diam. maj. 13-18, min. 12-16, alt. 131/2-18; Apert. lat. $7-8^{1 / 2}$, alt. $3^{1 / 2}-5$ mill.

Animal nigrescens, subtus pallidius, pes postice vix truncatus, supra cauda nigra, pro ratione parva, instructus.

Habitat in Camerunia: Bomana (D.); Vevoka (S.).

Specimina 34 exstant, omnia viventia collecta.

Cette nouvelle espèce paraît tout a fait isolée parmi le: autres que nous comnaissons. appartenant an genre Trochozonites. On ne retroure chez aucune autre ni la spire extrêmement prolongée à côtés concares. ni la sculpture singulière de la corquille, dont les 4 à ó demiers tom sont pliés d'une manière onduleuse fort caractéristique.

La sculpture embryonnaire et celle de la base, tout en arant l'apparence générale de celle du genre. nous semble pourtant en différer un peu, ce qui arec les antres particularités qu'elle présente, renr sa place parmi les Trochozonites assez douteuse.

Elle a été trouvée exclusirement sur une certaine espèce de fougère.

Nou, dedions cette charmante espece a MI. le Professenr H.ramer Thés, intendant du Mnsée loyal d'histuipe naturelle à Stockholm.

Il y a encore une petite coquille, conservée dans l'alcool, appartenant a rette famille. Elle se rapproche beanconp. par la torme. de Thapsia oleosa (Prentrer), mais en diftere frar la sculpture. Elle est revetue l'un ipiderme mat, nuance. colmes pale, et tonte sa surfare est converte d'une granulation extrinnment tine et gravin de stries an spirale plus distinctus sur la face opposée. Les tours de spire sont assez convexes. sripare par une suture prespue camalinulie. La rounille est percée d'un ombilic étroit, très profond. Son ouverture est lunaire et très étroite. Mesurant 4 mill. de diamètre maximum sur $3^{1 / 2}$ de diamètre min. et comptant $5^{1 / 2}$ tours de 
de spire l'espece ne parait pas itre complètement direloppée. L'animal, visible par la transparence de la connille, semble être unicolore, noir.

Probablement cette petite espèce appartient au genre Thapsia, mais son état incomplet nous détend de préciser sa place ou de lui proposer un nom.

Elle provient de Bomana, où M. Duséx l'a trouvée, à 1100. m. d'altitude, dans la mousse sur un trone d'arbre.

\section{Fam. HELICID $Æ$.}

\section{Genus Helix Linné.}

\section{Helix Camerunensis n. sp.}

(Pl. V, Fig. 11-13.)

Testa anguste et profunde umbilicata, depressa, epidermicle virescente-brumnea, apicem versus pallicliore, sericeo-nitidula, temui, facile deterenda, induta; spira depresse conica; antr. $4^{1 / 2}$, convexiusenli, embryonales lix rigati, ceteri oblique radiatim, irregulariter plicato-striati, striis spiralibus, snb lente dificillime distingnendis, dense ornati; sutura simplici. impressa discreti; nltimus supra peripheriam ticuia fusca cinctus, ad aperturam leviter ilescendens; apertura obliqua. late Innata; peristoma temp. breviter expansum. marginilus callo tenui junctis, supero planato, columellari superne subcalloso, triangulatim reflexo.

Diam. maj. 13, min. 10, alt. $7^{1 / 2}$ mill.

Habitat in Camerunia: Bibundi (S.).

Specimen unicum in museo reg. Holmiensi exstat.

Helix Jungneri n. sp.

(Pl. V, Fig, 14--16.)

Testa perrio-umbilicata, semiglohosa, epidermide virescentecornea, cereo-nitidula, temui, facile deterenda, induta; spira rotundato-conica; anfi. $4^{1} / 2$ (?), convexi, oblique radiatim, irregulariter plicato-costulati, striis spiralibus, sub lente vix perspicuis, densissime sculpti, sutura simplici, impressa dis- 
"reti: ultimus supura peripheriam pallide ringulatus. ad aperturam vix perspicue desendens; apertura lumato-cir'ularis, parum obliqua; peristoma tenue, breviter expansiusculum, margine columellari superne subcalloso, breviter r'eflexo.

Diam. maj. 13̈; min. 11, alt. $9^{1 / 2}$ (?) mill.

Habitat in Camerunia: Buea (J.).

Specimen unicum (apice destructo) in mus. zool. Univers. Upsaliensis exstat.

Si, en effet, les deux espèces que nous venons de décrire, aprortirment an genre Holix, rest la premiere toris, de motre sû, qu'on en a trouvé dans l'Afrique occidentale. Elles on l'une et l'autre le bord de l'ouverture réfléchi, trait caractéristique des Helix.

Chez la première, l'extrémité du pied ne présente, en outre, aucun pore muqueux; chez la seconde cette particulariti ne pent pas itre constate l'animal etant presplue entierement létruit.

Chaque espèce n'étant représentée que par un seul individu, nous n'avons pu examiner la radule, de crainte de briser la coquille en sortant l'animal.

Sous l'épiderme, qui se détache fort aisément, leur test est d'une nuance rose pâle qui devient blanchâtre vers le sommet.

Pour le reste, nous renvoyons aux diagnoses et aux figures y jointes.

Nous dédions cette espèce à M. le Dr. R. Junganer qui l'a déconverte.

\section{Rhachis Burnayi DoHrN.}

1866 Buliminus Burnayi (Rhachis) Domn Malak. Blätter p. 124. 'T. 5. Fig. $11-13$.

1868 Bulimus $\gg$ PFErFFer Mon. Hel. VI p. 45.

1881 Bulimina $》 \quad$ (Rachis) Prenfel Nomenclator p. 284.

Kaméroun: Bonge (D., S.); Balombi-ba-Kotta (D.). (Tlha do Principe.)

55 spécimens, tous recueillis à l'état vivant.

Les spécimens que nous avons examinés, ont tous deux fascies sur le dernier toux de la spire, la supérieure se trou- 
BIIANG TILL K. SY. VET.-AKAD. HANDL. BAND 22. AFD. IV. N:0 2. 59

vant immediatement au-dessons de l'angle obtus de la périphérie. Pas un n'était ni vitré ni d'une seule couleur. La coloration est en ontre agrémenté diun drois rangs de taches d'un brum rougratre, celles du rang inférienr etant les plus grandes, somvent sagittifumes, arec la pointe dirigre vers l'ouverture; sur les premiers tour's de la spire, ces taches se confondent quclquetois en formant des handes transrersales, onduleuses, un peu obliques, comme c'est le cas chez Buliminus electrinm (Hor.); parfois aussi elles disparaissent sur le dernier tou de la spire. Isa forme de la coupilie est yuelque peu variable, le dernier tour étant plus ou moins ventru et un seul individu correspond exactement à la figure qu'a donnée M. Reeve de B. nemricus.

Les uns ont l'apex coloré de la même teinte que le reste du test, les autres sont au contraire colorés, vers le sommet de la spire, d'un rose pâle, avec l'apex mêmie d'un pourpre foncé à peu prés noir. Les stries décurrentes sont ondulenses. La coquille est revêtue d'un épiderme très-mince d'une couleur paille pâle et dime apparence soyeuse; enfin les fascies et les taches sont, comme celles de B. electrinus, à moitié diaphanes et se reproduisent dans l'intérient de la corpuille avec autant de vivacité qu'au dehors.

Les oeufs, de forme sphérique, mesurent $1^{1 / 3} \mathrm{mill}$. de diamètre; il en fut trouvé environ 40 chez un individu.

L'espece a été recueillie sur les fenilles des Plantaniers et des Bananiers (MIsa sapientum et paradisiaca), et surtout sur celles du Cacaoyer (Theobroma cacao).

\section{Hapalus eminulus (MORELET).}

1848 Bulimus eminulus Morelet Revue zool. p. 353

1853

1858

1866

1868

1872

157

1881 Bulimina
Pfeiffer Mon. Hel: III p. 393.

Monelet Séries conch. I, p. 14, Pl. 1, Fig. 6.

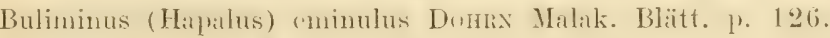

Bulimus eminulus Morelet Voy. Welw. p. 61.

Mus. Genova III p. 197.

Kaméroun: Loe (D.).

(Gabon; Angola: Loanda; Ilha do Principe (DoHrN); Abyssinie: Bogos, Rora-Beit-Andu). 
6 spécimens, tous recueillis à l'état vivant.

Les spécimens de cette espèce rapportés par MI. Dusḱx correspondent "xactement a la description et a la figure yrien

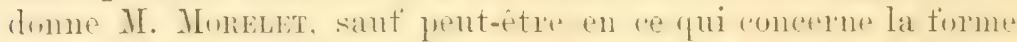

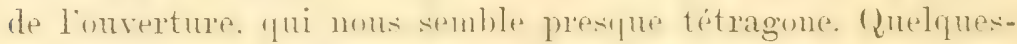
uns d'entre eux étaient couverts de terre. De même que dans les localités où elle a été trouvée auparavant, cette coquille est très rare à Kaméromn, n’étant rencontrée qu'à Loe, village éloigné d'environ 5 kilomètres du fleuve Massakke. Elle a été trourée rampant sur les feuilles de Koko (Colocasia antiquorum).

(\%) Hapalus sulcatus 11. sp.

(Pl. Y, fig. 5.)

Testa subfusiforme elongato-orata, imperforata, nitida, smberpendiculariter arruatim impupse suleata: spira apice obtusulo, elongato-conica; anfr. 7 , convexiusculi, sutura impressa, ad aperturam haud ascendente, marginata discreti;

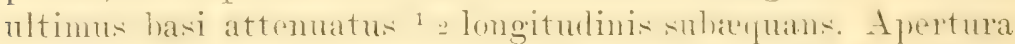
rerticalis sinuato-elongato-ovata: colnmella valde torta; peristoma rectum acutum, margine dextro sensim arcuato, medio modice arcuatim producto, columellari superne late reflexo, arluato, duplication'm formant", (alloso-incras.atu. subtus valde ad sinistram deviante, cum basali areuatim juncto.

Long. $8^{1} ;$ diam. $3^{1 / 3}$ mill.

Habitat in Camermia: Elimba-Liongo (D.); Buea (J.).

Specimina 3.

Ce n'est qu'avec la plus grande hésitation que nous plaçons eette monvelle repere dans le gente Hapalus, mais la ressemhande quelle mosente ave; Hapalus guineensis (.Inxas) (Philippi Abbild. I p. 54), provenant de même de l'Afrique occi-

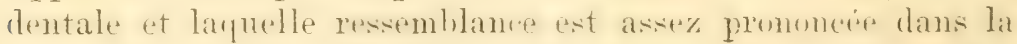
figure qu'a domnée M. ReEve (Conch. icon. Bul., Pl. 86, N:o 641), justifiera peut-être cela. Les diagnoses de ce genre nous semblent en outre assez contradictoires.

Les trois exemplaires que nous arons devant nous étant recueillis vides, nous n'avons pas pu examiner l'animal. 


\title{
Fam. ACHATINID $\nRightarrow$.
}

\author{
Genus Achatina Lamarck.
}

Achatina marginata Nwansox et var. gracilion v. IIARENs.

1820-21 Achatina marginata Swainson Zool. illustr. T. 30 ;

1831

1837

1839

1840

1848

1849

1849

1850

1855

1858

1860

1870

1876

1881

1882

1891
RANG in Ann. se. nat. XXIV p. 33.

Beck Index p. 75 N:o 2.

amphora JAY Catal. p. 58.

marginata Swainson Malacology p. 170, Fig. 23.

Pfimerer Mon. Hel. II p. 249.

ReEve Conch. icon. Pl. 4, N:o 14.

suturalis PHLLPp Abbild. III p. 29, Achatina T. 2, Fin. 1.

(Archachatina) AlBers Helicecn p. 190.

marginata AduMs Genera of rec. Moll. II p. 132.

Pfeiffer in Chemn. exl. II. Achatina N:0

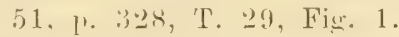

Monelé Séries conch. p. 19.

Albers Helicen ed. v. Martens p. 201.

var. gracilior

Semper Reisen im Arch. d. Philippinen, Landmoll. III p. 144.

var. I. Martens Monatsber. Berlin p. 257, 1. 2, Fic.. 1.

(Achatinus) PFeIFfer Nomenclator p. 264. v. Martens Jahrbüch. Mal. Gesellsch. p. 245.

Sitzungsbericht. Gesellsch. naturf. Freunde Berlin, Febr. 1891 p. 30.

Kaméroun: Massaka au district de Batanga, Mekango, Etome (D.); N'dian (S.).

(Victoria (B.), Barombi, Buea (Pr.).)

(Côte d'Or; Loango; Gabon etc.)

Douze spécimens de la grande forme; plus de 70 spécimens de la variété.

Parmi le grand nombre d'exemplaires de cette espèce que nous avons sous les yeux, il est possible de distinguer denx formes différentes. L'une, grande, mesurant jusqu'a 140 mill. de longueur et environ $70 \mathrm{mill}$. de largem, se distingue principalement par l'évidence de ses stries en spirale dans les 
tour's supérieurs. La conleur est d'un brun flammé, çà et là nettement violette, et se résolvant en points. Le sommet de la coquille et la columelle manquent le plus souvent de la conleur rose. L'onverture se déreloppe en forme de cloche, et le bord basal descend profondément sous la columelle.

La seconde forme, qui paraît être celle citée par II. r. Martexs comme une variété (Monatsber. l.c.), = var. gracilior v. Martexs (Albers Hel. l. c.), manque de la couleur violette et est moins nettement striée en spirale; les stries se résolvent fréquemment en granulations. Le bord extérieur est quelquefois faiblement ployé et le bord basal moins profond.

L'épiderme est d'm jamne paille clair', çà et là restant encore en place.

Ces deux formes varient très considérablement de forme, surtout la demière. Tantôt elles sont courtes et rentrues. tantôt étirées en longueur et étroites. Toutes deux ont les deux derniers tours couverts d'une sculpture très curieuse, composée de petites stries courtes qui se coupent obliquement. ot forment un risean à angles ohliynes diune excessive finesse. surtont ivilent sur les prints ou l'epillme est reste. Cette sculpture, qui manque sur l'avant-dernier tour, y est remplacée par des cordons en spirales se présentant ici sous la forme la plus apparente.

Tous les spécimens appartenant à la variété ont la columelle colorée soit d'un rose intense presque carminé, on d'un jaune rongeatre allant jusun'a nue teinte prespue dorée. La conleur du sommet de la coquille correspond en général ì celle de la columelle.

La columelle est très variable dans sa forme et dans sa troncature. La plupart des spécimens l'ont fortement arquée; (hez quelifues-mns, an contraire. elle est presifue absolument droite, et l'échancrure est tantôt abrupte, tantôt oblique.

Les flammules sont larges à la base et confluent fréquemment sous la périphérie. 'qui prend ainsi parfois une alplarence presque anguleuse, et il est meme posibible de constater un angle véritable chez une partie d'entre elles, surtout chez la grande forme.

Nous considérons avec M. v. Martexs qu'Achatina Cumingi sntтLwortu est synonyme dehatina marginata var. gratilior r. Mantexs, mais narant pas en l'oceasion d'en roir 
BIHANG TILL K. SV. VET.-AKAD. HANDL. BAND உ2. AFD. IV. X:0 2. 63

un exemplaire ou une figure, nous ne l'arons pas comprise dans la synonymie.

Cette espèce est très commune dans la région liamiromnienne; la grande forme parait pourtant être beancoup plus rare yue la variéte. M. Moremet dit qu'on la rencontre rampant sur le sol (Séries l. (.) mais tous les spéeimens que nous arous derant nous n'ont jamais été tronvés que sur le fenillage des arbustes et des arbres à nue assez grande hauteur. Elle dipose ses oenfis sur les arbres dans les angles des branches tout près du trone.

Cette grande espèce est avidement recherchée par les indigines, qui l'apprécient fort comme nomriture. М. S.rötin nous écrit sur ce sujet: On rencontre très fréquemment dans la forêt des négrillons portant une espèce de petites corbeilles tressees. d'une forme allongée. dans lesquelles ils la recreillent. Parfois ils pratiquent un trou rond dans la coquille à une faible distance de l'ouverture, et les portent enfilées sur. des lianes.

\section{Achatina Knorri Jonds.}

1839 Achatina Knorrii Jonas in Wiogm. Arch. I, p. 345.

1848 》 PFeirfer Mon. Hel. II p. 250.

1849 prunum Reeve Conch. icon. Pl. 4, N:o 13.

1853 Knorrii PFeIfFer Mon. Hel. III, 1\% 485.

1855 > $>$ in Chemn. ed. Kuster Achatina N:0

1881 105, p. 365, T. 47, Fig. 11, 12. Nomenclator p. 265.

Kaméroun: Etome (D.); Bonge (S.).

\section{0 spécimens.}

Les exemplaires dont nous disposons concordent partaitement tant arec la figure qu'avec la description d'Achatina prunum par M. ReEve, sauf en ce qu'ils sont un peu plus pointus. La supposition imise par M. r. MARTExs (Monatsber. 1. 255) que cette espèce se rapproche beancoup d'A. marginata Swarson est sans nul doute parfaitement motivée; cette espèce se distingue toutefois par certaines particularités qui l'en séparent. Telles sont, p. ex., les flammules nettement limitées d'un brun châtain très foncé sur fond jaune d'or, et la columelle très peu arquée et couleur de plomb, surtout vers sa partie supérieure. 


\section{Achatina Camerunensis 11. sp.}

(Pl. III, Fig. 1-4.)

Testa oblongo-ovata, temus, sericeo-nitidula, undique decuswatim. crebre ot distincte granulata, srannlis infra peripherian intertum infirmiorihns attamen clistinctis. olivaceolutea, strigis fuscu-castaneis, longitulinalibus, rarius fulguratis. intertum in masulis diswolutis in anfractu ultimo inf'ra peripheriam latiorihus et ad basin sope onflentihus oruata; spira conica, aprice obtuso, pallide rusto; sutura obsolete margrinata et suberemulata; antr. li. superi parum convexi. nltimus rentrous. "; longitudinis superans. basi dorso attenuatus: columella stricta, sinerne obsolete tortuso()-plicata. sursum wernlesens. allo allo-marginata, oblinge breviterque trun"ata. basin aprerture non attingens; apertura parmo olpliqua, olulongo-acuminato-ovalis, intus cornlesente-lactea, margaritacea, strigis externis transluentibus; peristoma simplex. acutum, siprius levisime expansum, intus fisco-limbatum, marginilus rallo tenuissimo junctis, dextro infra medium arcuatim prodncto.

Long. 80-90, diam. 40-50 mill.; apert. long. 47-53, lat. infr. merlium $25-28$ mill.

Habitat in Camerunia: Etome (230 m. alt., D.).

Specimina 50, quorum 3 in alcoh. serrata.

Cette espèce paraît se rapprocher le plus intimement

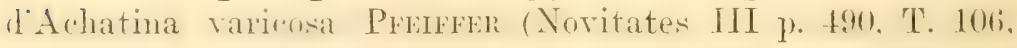
Fig. 1, 2) provenant du cap de Bonne Espérance, et à un "reptain degre d'A hatina fulgurata PFerffel ('Hexsitz el. Küster, Achatina N:0 37 p. 319, T. 26, Fig. 1), qui habite lifrique oceidentale, qroinuil soit rependant imposilule le la confondre avec elles.

La couleur et le dessin de ces espèces paraissent assez ressemhlants. mais che\% cette movelle espere, less flammules sont en général plus droites; ce n'est que vers la périphérie qu'elles oftrent partois la forme de zigzags. 
Le trait distinctif le plus caractéristique est tontefois la granulation qui recouvre la surface, mais qui s'affaiblit très legèrement au dernier tour au-dessous de la périphérie, tont ein restant parfaitement évidente. (hez les antres espèces an contraire, cette granulation cesse immédiatement audessous de la suture, et même elle y est très faible.

Fréquemment les flammules sont abruptement interrompues à la périphérie, ne se prolongeant vers le haut que par d'étroites ramifications domant une apparence anguleuse au dernier tour. A cela contribue anssi la circonstance qu'il $y$ court une série de granulations un peu plus longues.

Cette espèce présente aussi des varices peu marquées, particularité que l'on retrouve cependant che\% plusieurs autres espèces du genre, comme A. marginata, A. iostoma ete.

La suture est marginée et munie de crénelures fines, plus ou moins marquées.

La forme entière de la coquille est assez variable, comme on le voit par les denx exemplaires que nous arons reproduits, ce qui n'influe cependant sur la forme de la columelle, constamment droite, comme chez A. fulgurata, tandis que celle d'A. varicosa est assez arquée.

Le péristome, qui est bordé intérieurement d'un brum foncé, est quelquefois légèrement courbé en dehors.

\section{Achatina iostoma Pfeiffer.}

1852 1853 1855

Achatina iostoma Pfeiffer in Proc. Zool. Soc. London p. 86. Mon. Hel. III p. 485.

in Chemn. ed. Küster Achatina N:o 98 , p. 360 , T. 43 , Fig. 7.

Albers Heliceen ed. v. Martens p. 201.

balteata v. Martens Monatsber., Berlin, p. 258, T. 2, Fig. 2. iostoma Pfeiffer Nomenclator p. 265.

Kaméroun: Etome (à $230 \mathrm{~m}$. d'alt.), Bibundi (à $200 \mathrm{~m}$. d'alt.) (D.); Itoki Na N'Golo (S.); (Victoria (Buchноцz)).

(Fernando Po (Fraser).)

190 spécimens, dont 30 conservés dans l'alcool. 
Comme on le voit du reste par la synonymie qui prérède. cette espere ressemble beaucoup à $A$. balteata. Thi en l'occasion de me convaincre au Musée de Berlin que les exemplaires recueillis par M. Brcmolz a Victoria, dans le Kamérom, aprartiennent à Achatina iostoma Prerffer et non a A. balteata, qui n’a par conséquent pas encore été trouvé ì Kamẻroun.

La sculpture, la coulenr, le dessin sont les mêmes, et A. iostoma prisente également dans sa péripherie une fascie plus foncée, avec interruptions plus ou moins grandes.

Elle se distingue par la forme du dernier toux, plus allongé et diminuant rers la base, par la colnmelle presine droite et d'un bleu de plomb foncé rers le haut, et enfin par les Hammules plus serrées des tours superieurs, ainsi que par son test plus mince. L'intérieur de l'ouverture est d'un blane hentre, phos épais thez les grands exemplaires. Nons possédons cependant un tris grand exemplaire, mesurant 135) millimitres de longueur. dont la colmmelle est fortement tordue et arquée. en analogie de celle d'Achatina balteata, ce qui prouve le peu de valeur qu'on peut attacher à la forme de la columelle comme caractère spécifique.

On la rencontre à Etome. en compagnie de Gibbus Martensi Simru, frar multiturles sur le sol, non sur le feuillage des arbres, mais smlement dans une localité très restreinte. Elle s'y présente en si grandes quantités, que l'on rroirait marcher sur un tapis de coquilles d'oeufs». Elle habite aussi. frar individus relativement rares. la torêt vierge andessus de Bibundi, à environ 200 mètres d'altitude.

\section{Ganomidos nov. genus.}

Étymologie: yóvuru, vernis, cỉos, apparence.

'Testa ovato-eonica, tenuissima, sub-diaphana, nitidula, rornea, splendorem vernicis quasi imitans, strigis rufis ad suturam et ad peripheriam fulguratis vel antrorsum angulatim productis taenianque il,i efforientibus ornata, hic illic minutim albido-maculata; spira conica, apice mamillato, obtuso; anfr. circa 6 , "onvexinsculi, nucleo lavigato, confertissime chordato-plinati rel costulati et striis spiralibus subtiliter seulpti; 
embironales sutura canaliculata, plicata, ceteri sutura impressa discreti; ultimus inflatus, basi attenuatus, spiram sujerans; apertura obliqua, truncato-ovalis; peristoma simplex, tenue; columella leviter intorta, stricta vel modice arcuata, oblique truncata.

Animal nigro- vel fusco-strigatum et maculatum. Pes elongatus, sensim attenuatus, postice prope extremitatem supra triangulatim planatus, serrato-cristato-marginatus.

Ova elliptica, pro ratione parva, cretacea, sub lente crebre granulata.

Les deux espèces suivantes offrent, quant au test et à l'animal même, un si grand nombre de particularités les différenciant du genre Achatina, qu'il nous paraît impossible de les maintenir dans ce genre. Nous juoposons par' ronséruent de former pour elles le nonveau grenre que nous avons essayé de définir ci-dessus.

La première circonstance qui saute aux yeux, est la forme mamillaire du sommet, prorluite par la suture canaliculée, et spécialement sa sculpture, qui n'a pas l'apparence chagrinée différant de la sculpture générale du test, par laquelle se distingue le genre Achatina (voir Pl. III, Fig. 4).

En effet, le nucleus de la spire de ce nouveau genre manque de tonte sculpture, et le reste de la sculpture emliryomuaire est de la même apparence que celle du test en général.

La ténuité du test, mince. comme du papier, l'éclat gélatineux tout particulier, la sculpture verticale filiforme, et le système de la coloration, caractères qui s'accusent avec une parfaite évidence chez Ganomidos Shuttleworthi (PreIfFer), ne se retrouvent chez ancune autre espece positive d'Achatina à nous connue.

La circonstance que la columelle, tantôt droite tantôt arquée, est munie d'une échancurure pareille à celle du genre Achatina, et celle qu'à un examen superficiel le test possède une certaine ressemblance d'habitus avec ce genre, ne constituent pas, selon nous, une raison suffisante pour y faire entrer nue espèce qui présente ces caractères. En attachant une trop' grande importance à un caractère tel que l'échancrure de la columelle, le genre Achatina a recu une extension trop grande, et a fini par comprendre des genres qu'un 
simple coup d'oeil aurait dû interdire d'y rapporter. La présence de tous les carartères génériques. jointe à la ressomblance d'hahitus, est indispensahle pour justitier le classement d'espèces différentes sous un senl et même genre.

Il a été réservé à ces derniers temps d'opérer à cet égard un triage nécessaire, qui ne peut toutefois pas être encore consideré comme terminé.

Selon nous, les tours embryonnaires offrent des caractères de la plus haute valeur et les moins soumis de tous à des inthences exterienres et ocrasionnelles. ('es caractires embrymuares ont èté trop négligés juśrüici, probablement par la raison qu'ils exigent nécessairement un veil armé pour ctre parfaitement saisis, quoiqu'ils suftisent souvent à eux seuls pour décider la place d'une enpere donteuse dans lo système. Or, comme ces tours embryonnaires chez nos deux espèces se différencient de cenx du type Achatina tant par la forme que par la seulpture, cette seule circonstance pourrait justifier leur séparation de ce genre.

Même l'animal de ces deux espèces présente à l'extérien des différences qui justifient nltérienrement la formation d'un nouveau genre.

Le pied qui, chez les vraies Achatina, est court et large, est long rhez nos especes, tout en diminuant tries lentement de largeur. Le dos est aplati vers l'extrémité, et muni de denx areter laterales à hords dentelés, très andusées, et convergeant en arrière. On les retrouve, il est vrai, sous une forme tres faible, wez Achatina marginata swaxsox, mais ils font totalement défant diez A. iustoma Presfres. Non. profitons de cette oreasion pour demander s"il n'y aurait pas lien de séprarer $\mathrm{A}$. balteata REErE et les espéces qui se gronpent antour d'elle, telles que: A. instoma, pertecta. Banleirana, Paiverana, Tavaresiana ete, des Achatina décidement vraies, comme le centre desifuelles on placerait pent-être $\mathrm{A}$. marginati.

Le manteau tacheté de noir visible par transparence à travers le test. doit aussi etre considere comme une particnlarite de re genle (voir Pl. III. Fig. 9, représentant un animal retiré de la coquille et conservé dans l'alcool).

Les oeufs (Pl. III, Fig. 5), assez petits comparativement aux dimensions de la roruille, ont la blancheur de la craie, et présentent à la loupe de fines granulations. 
BIHANG TILL K. SV. VET.-AKAD. HANDL. BAND 22. AFD. IV. N:0 2. 69

La radule offre aussi des différences. La dent centrale n'est ni aussi courte, ni anssi étroite relativement anx dents latérales que p. ex. chez A. marginata. Les différences que présentent la radule de ce nouvean genre comparée ì celle de l'Achatina marginata résultent d'ailleur's des tigures cijointes.

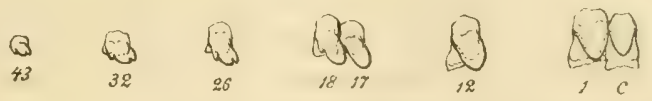

Radule de Ganomidos Schuttlenorthi (PFEIfFer). c, dent centrale; 1, 12, 17, 14 dents latérales, 26, 3:2, 43 dents marginales du côté gauche; les chifrres indiquent l'ordre numérique à partir de la dent centrale.

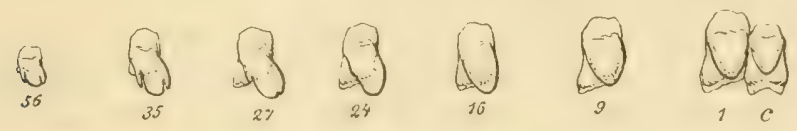

Ratule de Ganomidos Barrianum (Sowerns). e, dent centrale: 1, 4, 16, 24 dents latérales, 27, 35, 56 dents marginales.
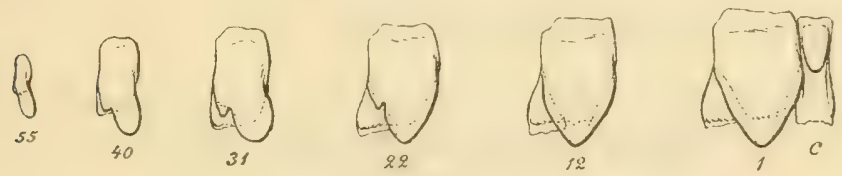

Radule d'Achatina marginata Swarnson. c, dent centrale; 1, 12, 22 dents latérales, $31,40,55$ dents marginales.

\section{Ganomidos Shuttleworthi (PFEIFFER).}

(PI. III, Fig. 11-14.)

1856 Achatina Shuttleworthi PFerforer in Proc. zool. Soc. London p. 34.

1859 \

Mon. Hel. IV p. 603.

$1881 \quad \gg \quad$ (Achatinus) Pfeiffer Nomenclator p. 265.

Kaméroun: Bonge (S.), N'dian (D., S.).

(Grand Bassam (Verreaux).)

15 spécimens, dont 11 conservés dans l'alcool.

La diagnose que M. Pferffer a donnée de cette espèce ne mentionne point les taches d'un jame pâle blanchâtre très 
distinctement aceusées of domnant is la corpuille mu fort hel aspect. Elles présentent me apparence émaillie, oprarpur. w se tronvent rhez Ganomidos Shuttleworthi presule exrhusive ment derant la pointe des taches hrunes sagittiformes. yni constituent à la périphérie et près de la suture les deux ceintures raracteristipues. Flles sont produites par la transformation de la substance des coites mêmes formant la sonlpture transversale, tandis que le fond du test visible entre elles a conservé la couleur du reste de la coquille. La surface est souvent corrodée dans ces taches.

La sculpture transversale est chez cette espèce très fortement aucusée et régulière. tandis que les stries an suiralus sont extrêmement fines.

Le plus grand de nos spécimens ne mesure que 27 mill. de longuen sur $1 t^{1}$ a de diametre; il est ainsi considerablement plus petit que le type de M. Prasrer. qui mesure une longueur de 34 mill. sur 17 de diamètre.

Comme l'espèce suivante, Granomidos shuttleworthi vit. en petit nombre dans des endroits sombres au pied des souches, sous l'écorce détachée, etc.

\section{Ganomidos Barrianum (SowERBI).}

(Pl. III, Fig. 5-9.)

1889 Achatina Barriana G. B. Sowerbi in Proc. zool. Soc., London 1. 57!. Pl. 5ti, Fie. 2.

$1891 》 \quad$ v. Martens in Sitzungsber. Gesellsch. naturf. Freunde, Berlin, Febr. p. 30.

Kaméroun: Bonge (D., S.); Itoki, Kitta (S.); Bibundi (J.); (Barombi (PR.)).

(Calabar.)

36 spécimens, dont 34 conservés dans l'alcool.

Les stries en spirales sont heancoup plus marques che\% cette esperce, tandis que la sculpture transrersale n'est ni aussi fortement acousée, ni anssi réguliere que chez la précédente. (r qui donne jusqu'à un certain point à la coquille une apparence plaquetée (voir Pl. III, Fig. 7).

Le dessin est, de même, beaucoup moins distinct, et la roquille etant beaucoup plus grande. les taches blanchatres 
sont toutefois très petites, disséminées sans nul ordre sur toute la surface. A cette exception près, ces taches sont de la même nature.

Le type qu'a décrit M. Sowerby n'a pas, à beaucoup près, atteint son déreloppement complet, ne mesurant que 43 mill. de longueur sur 23 de diamètre. ()r nous possédons des exemplaires qui atteignent jusqu'à 59 mill. de longueur sur 333 de diamètre. L'espèce est, d'ailleurs, d'une taille très variable, la spire étant plus on moins allongée, et le dernier tour étant plus ou moins renflé. La columelle est ou droite, on médiocrement arquée. Les oeuts mesurent $6^{1} 2$ à 7 mill. de longneur sur 5 de diametre; un individu en contenait 14.

La figure colorée yu'a jointe à sa diagnose M. SowerbY ne dome pas une idée parfaitement exacte de la physionomie de l'espèce.

\section{Genus Petitia Jousseaume.}

\section{Petitia pulchella (v. Martens).}

1876 Achatina pulchella v. Martens Monatsber., Berlin p. 258, T. 3, Fig. 1, 2.

1884 Petitia petitia Jousseaume Bulletin de la Soc. Zool. de France p. 172, Pl. 4, Fig. 4 a.

1889 Achatina Smithi Sowerby in Proc. zool. Soc. London p. 579, Pl. 56 , Fig.. 3.

Kamérom: Bibundi (J.); (Bonjongo, Victoria (B.); Buea (Pr.)).

(Congo (Petit)).

5 spécimens.

Il résulte avee toute évidence, non-seulement des diagnoses, mais aussi des figures, que l'Achatina Smithi de Sowerby et la Petitia petitia de M. Jousseaune appartiennent à la mème espèce qu'a décrite et figurée, déja en 1876, M. v. Martens sous le nom indiqué plus haut. La seule différence que présentent les diagnoses se rapporte à la forme de la columelle, M. Sowerby l'indiquant droite, M. Jousseaune presque droite, et M. v. Martens la décrivant comme arquée. La forme de la columelle est dès lors sans nul donte variable chez cette espèce, et 
le fait que cette dift"rence ne préjudicie en rien it lidentiti des deux especes résulte. it nén pas douter. des spécimens que nous arons sous les yeux (dont un type, que M. v. Marters a eu la libéralité de me présenter), qui ont tous la columelle droite (=Achatina smithi sow.), comme la semble indiquer aussi la figure, donnée par M. V. Martens.

Les tours embryonnaires sont parfaitement lisses. Les stries spirales. trés serrées. du reste de la coquille sont légèrement onduleuses.

Les oenfs sont d'une forme elliptique très obtuse, et mesurent 5 mill. de longueur sur 4 de largeur. Ils sont, à en juger par deux qui ont été tromvés dans nue compluille vide. d'me nuance blanche sale, à surface inégale.

Xons supposons que l'Achatina specularis de Morilet (Toyage Wruwtsch p. 74. Pl. 4, Fig. 4) 'pui n'est guère me vraie Achatina, dans le sens nous domnons à ce genre, est tres voisine de Petitia pulchella, mais nous ne samrions an contraile partager lopinion de M. v. Martexs sur l'aftinité de l'Arhatina zebriolata de Monktex (ibirlem p. 72, Pl. 3. Fig. 1).

\section{Genus Limicolaria Schumacher.}

\section{Limicolaria rubicunda Shuttleworth.}

1856 Limicolaria rubicunda Shuttleworte Notitiæ malac. p. 45. 'T. 7. Fig. 4, 5 (excl. syn. Reeve).

$1859 \gg \quad$ Pfeiffer Mon. Hel. IV p. 583.

1860 Achatina $\gg$ (Limicolaria) Albers Hel. ed. v. Martens p. 198.

$1869 》$ 》 $》$ v. MARTENS in Mal. Blätt. 1. 73.

1876 Limicolaria 》 v. MLarTens Monatsber. Berlin p. 258. 'T'. 3. Fin. + (\%).

$1881>\quad$ P Pfeiffer Nomenclator p. 262.

1895 》 KonelT in Chemn. ed. Kob.p. 125 T.35, Fig. 2-5; (T. A, Fig. 2?).

Kaméroun: Bibundi (D.. .'., I.); Bonge (D).). (? Vietoria (B.).)

(Kissy.)

34 spécimens, dont 4 conservés dans l'alcool. 
Parmi les individus que nous avons examines, s'en trouve un qui mesure 49 mill. et demi de longueur sur. 20 mill. de diamètre. La forme de la columelle est très variable, étant plus ou moins tordue et plus ou moins arquée.

M. Kobent n'a pas cité dans la synonymie l'espéce qu'a figurée ML. Reeve (Conch. icon. P1. 53. N:o 352) sous le nom de Bulimus flammeus, et ce n'est qu'avec un point d'interrogation que M. Shutrueworti l'a déclarée identique à son espèce; elle présente en effet trop de différences pour qu'elle puisse être considérée comme identique à Limicolaria rubicunda. Elle manque p. ex. totalement du dessin caractéristique, c'est-à-dire des Hammules descendant de la suture entre les grandes flammes qui s'avancent d'en bas; la grandenr et la forme de la coquille, de même que celle de la columelle, sont également très différentes.

C'est aussi avec hésitation que nous reconnaissons une vraie L. rubicunda dans l'espèce dont M. v. MLrTess a domé une figure reproduite par M. KовеLT (1. с. T. A, Fig. 2); cette espèce, ainsi an'un de nos sujets (mesurant 46 mill. de longueur et provenant de Bonge), appartiendraient-ils par' hasard à la variété ou à l'espèce distincte, que M. ShuttusworTu décrit par res mots: discrepant nempe a typo, testa forsan majore, albida, strigis latioribus atro-castaneis, et columella minus torta, margine latius revoluto, qui peuvent être appliqués mot à mot à notre spécimen? La columelle forme en outre un angle distinct avec le bord basal, et la perforation est plus large. La circonférence de la spire est plus convexe et le dernier tour plus ventru. Si, après l'examen de plusieurs individus, on trouvait que ces particularités autorisent la création d'une nonvelle espèce, nous juroposerions pour elle le nom de Limicolaria shuttleworthi.

Limicolaria rubicmda à été trouvée en grande abondance dans les environs de Bibundi, ou elle vit sur Pennisetum polystachyon, sur des plantes grimpantes, des plantaniers etr., ainsi que sur le sol.

\section{Limicolaria felina SHuttLeworth.}

1856 Limicolaria felina SHUT'Lewortu Notitiæ malac. I p. 47, T. 6 , Fig. $5,6$. 
1860 Achatina felina (Limicolaria) Albers Heliceen ed. v. Martens 1). 198.

1881 Limicolaria 2 PFeIfFer Nomenclator p. 263.

1893 » KobelT in Chemn. ed. Koв. p. 54, T. 17, Fig. 6, 7.

Kaméroun: Bibundi (D., S.), Bonge (D.).

(Côte d'Or: Abetifi, Grand Bassam. Gabon.)

25 spécimens, recueillis tous à l'état vivant.

Cette espèce nous paraît très voisine de la précedente, avec laquelle on la reneontre, et parmi les spécimens qui sont a notre disposition. se trouvent des formes dont nour ne saurions préciser la place. Nous ne serions pas surpris si. apres un examen rigoureux, ces denx esperes étaient reommes simplement romme des formes on des rariétés de la même espece. glissant insensiblement l'une dans l'antre.

\section{Limicolaria tenebrica (REEve).}

1848 Bulimus tenebricus ReEve Conch. icon. Pl. 53, N:o 347.

$1853 \gg$ Pfeiffer Mon. Hel. III. p. 387.

1855 Limicolaria tenebrica Adams H. et A. Gen, of rec. Moll. p. 133.

$1856 》 \gg$ Shutrleworth Notitiæ malne. p. 50.

$1859 》 \quad$ Pfeiffer Mon. Hel. IV. p. 585.

8160 Achatina $\quad$ (Limicolaria) Albers Heliceen ed. v. Martens p. 198.

1881 Limicolaria 》 Pferffer Nomenclator p. 263.

1894 ॠ $\gg$ KовLT in Chemn. ed. Kов. p. 66 T. 20, Fis. 7 , s.

Kaméroun: Ekumba-Liongo (D.).

(Ibu, Grand Bassam.)

(L'Afrique centrale (Baker fide H. Adans).)

Un seul spécimen se trouve au Musée d'hist. nat. à Stockholm.

L'unique individu que noms ayons devant nous, est color' d'un brun rougeatre, et s'accorde mienx avec la figure quen a domée M. Risere, dont la couleur est pourtant plus rose. qu'avec celle que donne M. Kobert, qui est d'une teinte jaunâtre. 
Quoique nayant pas atteint son complet développement. il est néanmoins de la même longueur que le type de $\mathbf{M}$. Kobet, mais un peu plus large. La circonférence de la spire est, en outre, un peu plus convexe.

L'hésitation que montre M. KobeLt à réunir son espèce avec Limicolaria tenebrica (REEve), nous semble parfaitement fondée, car ce n'ent quarec la plus grande peine qu'il serait possible de recomnâ̂tre la même espèce dans les deux figures.

\section{Limicolaria Numidica (REEVE).}

1848 Bulimus Numidicus Reeve Conch. icon. Vol. V, Pl. 53 N:0 351. $1853 \gg$ PFeIfFer Mon. Hel. III p. 386.

1855 Limicolaria Numidica Adaus H. et A. Gen. of rec. Moll. p. 133. $1856 \gg$ ShutTleworth Notitiæ malac. p. 44.

1858 Bulimus tlammeus Moreiet Séries conch. p. 17.

1859 Limicolaria Numidica Pferffer Mon. Hel. IV. p. 583.

1860 Achatina $》$ (Limicolaria) Albens Heliceen ed. V. MARTENS p. 197.

1866

v. Mar'tens Malak. Blätter, B. 13, p. 105 , 'T. 4, Fig. $5-8$.

1874 Limicolaria flammen rar. numidica Jickelr Moll. Nord-Ost-Afr. p. 159 .

1877

1881

1894 flammata Pfeiffer Mon. Hel. VIII. p. 269.

》. Nomenclator p. 262.

Numidica KobetT in Chemn. ed. KoB, p. 75 T. 12 , Fig. 7. 8; T'. 25. Fis. 3-8.

$\beta$ pallide rufo-picta.

$\gamma$ unicolor, pallida (=var. $\beta$, Shuttleworth).

Kaméroun: Bonge, Bibundi (D., S.); Loe, Boangolo, Ekumba-Liongo (D.); Itoki (S.).

(Senegal; Yoruba on Arriba (MAxx.); Gabon; Semaar.)

Plus de 350 spécimens, dont environ 200) appartiennent a la variété $\beta$. La plupart ont été recueillis a l'état virant.

Cette espéce parait être la plus répandne à Kaméroun, de même que la plus riche en individus. La variété $\beta$, à dessin brun clair, semble la plus commune.

Elle varie considérablement dans son habitus, sa couleur. sa sculpture et la forme spéciale de la columelle. La forme 
grimirale pent itre plus on moins allongée; la conlenr passe de lincolore par des variations insensibles au brun clair et an brun rhâtain fonce, et la somlpture varie d'une reiticulation fortement prononcese ot régulière a labsence de tonte rétirnlation. La "rénelure et la margination de la suture sont de même tris rariables. La eonsistance et lépaisseur du trst présentent de tris grandes variations. Ainsi, p. ex., les exemplaires clairs et les exemplaires incolores de Bonge sont excessivement fragiles, et bien que partaitement adultes et munis d'oeufs, minces comme du papier.

Dans la plupart des cas, la columelle est parfaitement droite, mais chez un certain nombe dexemplaires. plle offre, i la base, me déviation remarqual)le it gamble. Elle est tonjour's d'un violet intense chez les exemplaires a dessin fonce, mais chez les varietés claires, elle passe du chair clair a me décoloration romplète. Le trait aractéristipue du dessin est la lifureation en fourchette des Hammes a leur sommet. ce "pui proluit firinemment, surtout ahez les exemplaires à robe foncere, la formation d'un srstemede stries etroites immédiatement au-dessous de la suture. Une autre particularité de dessin. dejat signalée par M. Y. Mlartws (Mal. Bl. 1. с.). mais yui prait toutefois itre commune a plusienrs espèces du genre, se trouve dans le changement subit de direction des flammes anx tours inferreurs: elles prement sons la périphérie une direstion plus on moins horizontale et deviennent rubances en romplete conformiti aver Achatina polyohroa Morelet (Voy. Welwitsch, p. 72, Pl. 3, Fig. 5). Chez les exemplaires achulter, re processus "aracteristinge les thammes n'existe pas sur la totalite de la contulle. mais resse an demier tour, ou la dernière période de croissance oftre nu proressus normal des flammes.

Certe esprece est généralement indiquée comme rampant sur le sol. mais la plupart des exemplaires actuels ont été recueillis sur des plantes, surtout sur Canna indica L., et sur los fenilles des plantanims (Musa silpientum et paradisiaca L.).

Les oenf's, d'un jaume pâle, ont une longueur de 4 mill. sur une largeur de :31 "s mill. Th "xemplaire 'n contenait 25.

Un individu qui ne possède pas encore toute sa croissance a des petits parfaitement developues.s. avec 4 tours, d'ou l'on doit inférer que l'espèce pent être ovo-vivipare. La 
jemne coquille est foumie is sa périplérie d'un angle tris évident.

Nous n'avous pas en à notre disposition l'ourrage de Mi. CAImbado, T'oyage à Méroé, et par ronséquent nous n'arous done cité son espèce Helix flammata dans la synonymie. Le Bulimus Kambeul yuont figuré MII. Potrez et Mrchacd (Galérie Douai p. 145, Pl. XIII, Eig. 11, 12) semble différer trop dans le dessin, pour qu'on puisse le considérer comme synonyme de Limicolaria Numidica (ReEve).

\section{Limicolaria Aurora (JAY).}

1839 Bulimus Aurora JAY Catalogue p. 119 T. 6 Fig. 2.

$1848 》 \gg$ Pfelfeer Mon. Hel. Il p. 199. Adansoni $\gamma \gg \gg \gg \gg 179$.

$\gg$ Adansoni $\gamma \gg \gg \gg$ 》 $\gg 179$.

$\gg$ suffusus Reeve Conch. icon. Vol. V. Pl. 53 N:o 350.

1853 \ Aurora Peeifier Mon. Hel. III p. 385.

1855 Limicolaria $\gg$ ADAMS H. et A. Gen. of. rec. Moll. p. 133. suffusa

1856 Aurora Shutreeworth Notitia malac. I. p. 49.

1859 》 PFelfFer Mon. Hel. IY p. 585.

1860 Achatina $\gg$ (Limicolaria) Albers Hel. ed. v. MIartens p. 198.

1874 Limicolaria flammea var. JickeLI Moll. Nord-Ost-Afr. p. 157.

1876 》 Aurora v. Mar'ens Monatsber. Berlin, p. 258.

1881 》 PFeIfFer Nomenclator p. 263.

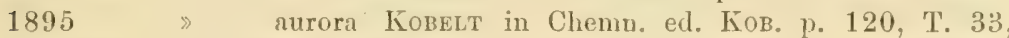
Fig. 5, 6.

Kaméroun: Ekumba-Liongo, Boangolo (D.); Bonge (T)., S.); Bibundi, Boana, Buea, Victoria (J.).

(de Senegal à Gabon.)

Plus de 100 spécimens, recueillis à l'état vivant.

Nous ne doutons pas que l'on ne pût créer sans difficulté au moins trois especes, dans la masse d'exemplaires que nou: avons été forcé de rapporter à cette espèce-ci, et nous nous tromperions fort s'il n'était pas possible de mettre dans ce nombre Limicolaria striatula (MÜLLER). C'ette dernière doit. à ce que l'on dit, se distinguer spécialement de Limicolaria Aurora par une forme plus cylindrique, une sculpture spirale plus accusée et la forme de la columelle. Le fait que L. striatula est une très mauvaise espèce, résulte entre autres des figures données par M. SHuttheworti (Notitiæ malac. 
T. VIIT, Fig. 1) et par M. Kobelt (in Chemn. ed. Kobelt, 'T. :3.) Fig. :3. 4), "ar elles paraissent difticilement ponvoil' representer la même expèe.e. La stronymie domnée prar ces auteurs parait aussi legitimes rette maniere de voir. Lia tignre? de la planche 141 de l'Histoire naturelle des Mollus-

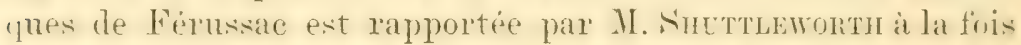
a L. striatula et a L. Aurora, cette dernière rependant avee un point d'interrogation, et M. Kónist plave sous L. striatula

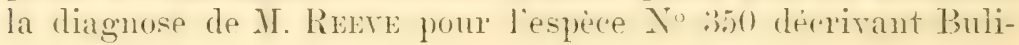
mus suffusus (= Limicolaria Aurora), et sous L. Aurora la figure appartenant à la même diagnose.

Limicularia spectralis PrenFen est pent-itre igalement me espèce douteuse pareille, sous laquelle se cache ou L. Anrora on la forme incolore de L. Numidica. Nous posivedoms des exemplaires de $\mathrm{L}$. Aurora qui, concordant an reste en tont. se distinguent cependant par la fiomme de la colnmelle, parfaitement droite che\% les uns, tris arquie chez d'autres, en ceci qu'elle dévie à gauche vers le bas.

Même la sculpture varie en intensité, et la forme totale passe d'exemplaires assez ventrus à des exemplaires minces, plus on mons rylindriques. Ises exemplaires venant de Virtoria et de Boana sont plus courts et plus ventrus que les antres. et paraissent par consisuent appartenir ì la même forme que relle rermeillie par M. le protesseur Bronnos\%. La "ouleur est ansi variable. La teinte rougeâtre a laquell" on doit l'origine des noms d'Aurora et de suffiusa, est fréquemment remplacee par la teinte d'm blanc pur sous un épiderme jaunâtre indiquée pour I. striatula.

Nous risquerons donc la supposition que res denx esperes nien font qu une en réalité, et le manque seul d'exemplaires typiques pour comparaison noms a empeché de comprendre Limicolaria striatula (MithLen) dans la sprnonymie domée cidessus.

()n los rencontre principalenent sur les fenilles de Canna inclica (tontes res roquilles sont alors lougeatres) et sur les

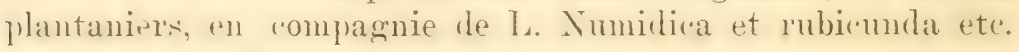




\section{Limicolaria Kobelti n. sp.}

Testa aperte et profunde perforata, oblongo-ovata, subpellucens sed solidiuscula, irregulariter leviterque plicatostriatula, nitidnla, in anfractibus intermediis infra suturam striis pancis, spiralibus, sub lente vix perspicuis, decussata, corneo-lutescens, apicem unicolorem versus subrosea, strigis et flammis atro-castaneis, siepius obliquis, in anfractibus superis linearibus, in ultimo flexuosis, ad basin siepe confluentibus, ornata. Spira convexo-conica, apice obtuso; anfr. 7 , convexiusculi. sutura pallida, subtiliter cremulata et plicata, linea impressa anguste marginata, discreti; ultimus postice spiram paululum brevior, rotundatus, medio obsolete planatus, strigis infra medium flexuosis, angulum simulantibus ornatus, sed interdum re vera obsolete angulatus, angulo aperturam versus evanescente, basi circa perforationem in carinam distinctam compressus. Apertura fere verticalis, angulatoelliptica, intus albido-coemulescens, strigis extemis pellucentibus; columella leviter areuata, vix torta, violacea; peristoma rectum, angustissime hadio-limbatum, marginibus callo tenuissimo junctis, dextro regulariter arcuato, in medio leviter arenatim producto, columellari longius, plane, vix formicatim reflexo, cum basali angulum distinctum, fere canaliculatum, carinam externam respondentem, formante.

Specimen maximum: long. 411/2; diam. maj. 20, min. 17; apert. long. 17, lat. 9 mill.

minimum: long. 33 ; diam. maj. 16, min. 151/s; apert. long. 15, lat. 8 mill.

Ova longa 4, lata 3 mill.

\section{Habitat in Camerunia: Bonge (D.).}

Specimina 46, viventia collecta, in coll. mea exstant.

Les difficultés que rencontre la précision d'une espèce dans ce genre sont unanimement reconmues de tous ceux qui ont eu à s'en occuper, et c'est par conséquent chose toujours très délicate que d'y établir une nouvelle espèce. Après étude scrupuleuse de tontes les descriptions et de toutes les figures publiées jusqu'ici sur ce genre protéen, il nous a 
tomtefois eté imposible dy ranger, parmi les espèeses actuellement connues, cette petite Limicolaria, remaryuable par sa beanté.

Ce qu'il y a de plus marquant chez elle, c'est la perforation iminemment ourerte, d'une grandenr pen commune pour de si petites espéces du genre. et l'angle caréniforme qui l'entoure. Dans la rígle le bord columellaire. replié en général en cornet, recouvre la perforation en ne laissant lihre yu'me fente étroite. Chez notre espèce-ci. an contraire, (e) bord est simplement aplati et non enronlé. laissant toute la perforation à peu près libre.

Nous n'avons trouvé dans aucune autre espèce que nous arons en l'occasion d'examiner, me pareille alsence presifue totale de la sculpture spirale, du reste assez commune et plus ou moins marque du moins chez les tours de spire médians. C'hez tous nos exemplaires elle est invisible a l'oeil nu, at chez quelques-uns il est à peine possible de la déconvrir même sous grossissement. Elle n'occupe en outre que la région située immédiatement sous la suture.

Vient ensuite l'éclat tout particulier, presque huileux, dépendant en partie de cette absence de sculpture chez la coyuille et qui nous semble de mème être quelque rhose de spécial pour notre espèce.

Dans son habitus général, elle parait se rapprocher beaucoup de plusieurs espèces telles que Limicolaria tenebrica (Risere), Is. martensiana Surtu, L. Henglini v. Martexs et L. jaspidea (Morelet), mais elle offre néanmoins des particularités qui la distinguent de chacme de ces especes. Ainsi, elle diverge: de tenebrica par son ton fondamental jame de corne, par son dessin transparent dans l'intérieur, par un tour de spire de moins, et par sa columelle aryué de martensiana par sa perforation ouverte, et par sa carene particulierement éridente entourant la perforation et produisant l'angle qui se roit au passage de la columelle dans le bord hasal; elle se distingue anssi par ce dernier caractère de L. Heuglini; la tigure donnée par Jickeli pour rette espiese (Moll. Nord-()st-Atrika's 'T'. TI, Fig. 10) rapelle beancomp ]a notre, qui néanmoins a les flammes généralement plus larges et plus flexueuses, ainsi qu'une spire moins étirée.

Par son systeme de coloration, elle se rapproche sensiblement de L. jaspidea (MorELET), telle que cette dernière a été 
reproduite dans le Voyage de Weuwissch, Pl. II, Fig. 2 (l'exemplaire à couleur foncée). Une autre ressemblance avec la même espèce est celle que le dernier tour de spire présente un angle obtus très évident chez quelques exemplaires, mais qui disparait cependant toujours vers l'ouverture. En analogie avec ce que l'on voit chez L. martensiana, les flammes produisent aussi, par leur flexuosité et par leur changement de direction à la périphérie, une apparence anguleuse, de sorte que les exemplaires, quoique manquant à peu près d'un angle réel, paraissent néanmoins anguleux. Cet angle périphérique est parfois visible très haut sur la spire sous la forme d'un renflement immédiatement au-dessus de la suture.

Les flammes, qui sont d'un noir pourpré, la teinte rougeâtre étant plus prononcée que chez L. jaspidea, rayonnent d'ordinaire depuis la région ombilicale, et présentent d'ordinaire leur plus grande largeur au milieu, mais confluent parfois en grandes taches, de temps à autre jusqu'à recouvrir toute la partie du tour de spire située sous la périphérie.

La suture finement crénelée et plissée se retrouve entre autres chez L. rubicunda, et la ligne imprimée qui l'accompagne, chez L. martensiana.

La spire est en général moins allongée que chez les espèces indiquées, et le dernier tour de spire, mesuré à son côté postérieur, est de bien pen inférieur à la hauteur de la spire, et légèrement ventru à la base.

La columelle faiblement arquée est tonjours violette, et l'intérieur de l'onverture est recouvert d'un callosité blancbleuâtre, qui n'empêche pas, cependant, le dessiu extérieur de paraître parfaitement à travers. Cette converture s'épaissit vers le péristome étroitement bordé d'un brun clair, et forme un mince callus immédiatement en dedans de ce bord.

Cette coquille n'a été trouvée que par M. Dusḱn et uniquement à Bonge, où on la rencontre tant sur le sol que sur les feuilles des Plantaniers.

Nous dédions cette belle espèce à M. Konflt, l'éminent conchyliologiste à qui l'on doit une monographie spéciale de ce genre difficile. 


\section{Genus Perideris Shuttleworth.}

\section{Perideris Solimana (MORELET).}

1848 Bulimus Solimanus Monelet Revuc zool. p. 353.

1851 suturalis PferfFer Proc. zool. Soc. London p. $255 \mathrm{Pl}$ 2, Fig. 2.

Achatina Sillimani Deshayes in Fér. Hist. II p. $152 \mathrm{Pl} .137$, Fig. 14, 15.

Solimana PeTí in Journ. de Conch. 'T. 2 p. 267, Pl. 8, Fig. 8.

1853 Bulimus Solimanus Pferffer Mon. Hel. III p. 299.

Sillimani $\gg$ in Chemn. cd. Küster p. 88, 'T' 31, Fiug. 7, 8 .

1855 Limicolaria suturalis ADAMS Genera of rec. Moll. II. p. 133. Pseudotrochus Solimanus $\gg \gg 》 \quad 》 \quad$ p. 135.

1856 Perideris Solimana Shuttieworth Notitia malac. p. 78.

1858 Achatina 》 Moreiet Séries conch. p. 23, Pl. 2, Fig. 2. 1859 Perideris $>$ Pfelffer Mon. Hel. IV p. 593.

$1860 \gg$ Albers Heliceen ed. v. Martens p. 205.

1876 \ $\gg$ Martens Monatsber. Berlin p. 259, 'T. 3, Fig. 3.

1881 》 $\gg$ PeEIfFer Nomenclator p. 261.

$1891 》$ V. MARTENs Silzungsber. Gesellsch. naturf. Freunde, Berlin, Febr. p. 31.

$1893 \gg$ KoвLLT in Chemn. ed. Ков. p. 32. T. 10, Fig. 2, 3; 'T. 16, Fig. 4, 5 .

Kaméroun: Bomana (à $66010 \mathrm{~m}$. d'alt.. D.); Kitta, N'dian, Itoki (S.); (Victoria (B.); Barombi (Pr.)).

(Gabon.)

10 spécimens, dont 5 recueillis à l'état vivant.

Les fascies du dernier tour sont assez variables d'éclat, de couleur et de largeur, mais l'inférienre est tonjours plus foncée et plus large que la supérienre, qui tantot se perd sous la suture, tantot s'apercoit assez haut sur la spire. La supérieure se trouve sur l'angle dn dernier tour, et linférieure tient presque le milien entre la première et la columelle, ou s'approche un peu de celle-ci. L'inférieure qui n'atteint pas le péristome, est située à l’intérieur du test, n’étant visible à l'extérieur que par transparence. A cet égard, la figure qui en a été donnée par M. Monelet (Séries I. c.) n'est pas 
complètement exarte; en outre la columelle y est trop rouge.

Chez un de nos exemplaires, la fascie supérieure, qui se tronve à l'extérieur du test et qui a toujours la finesse d'm fil, n'atteint pas non plus complètement le péristone. Les fascies pénètrent dans la substance du test, et elles ne sont donc nullement épidermoïdales comme l'indiquent MII. RANo et DoIrns chez. Perideris alabaster (RANG). Mais l'épiderme est souvent, sur l'angle du dernier tour, d'une nuance jaunâtre, d'où résulte une sorte de bande superficielle, au milieu de laquelle on voit par transparence la rraie fascie de la périphérie. Chez un senl exemplaire la coloration consiste senlement en rette bande secondaire, les deux fascies manymant parfaitement, et en me nuance d'un brun pâle sur la columelle.

Le test de cette espèce est plutôt solide, comme le dérit M. Monelet, que mince et faible comme le prétend $M$. Pfeiffer. Les bords de l'ouverture sont toujours réumis par une callosité très mince et incolore. Chez un seul de nos individus, la hauteur du dernier tour dépasse celle de la spire. Le plus grand exemplaive mesure 40 mill. de longueur sur 23 de diamètre.

Les oeufs, dont 14 furent trouvés dans un individu de 30 mill. de longueur, mesurent 5 mill. de longueur sur $3^{3 / 4}$ de diamètre. Ils sont polis et d'une blancheur éclatante.

Les figures de cette espèce dans Chemnitz, l'édition de Küster (1. c.), sont aussi très incorrectes: la forme papillaire de l'apex, trait générique tout particulièrement caractéristique, n'y est pas marquée, et la couleur de la fascie inférieure, qui est d'un bleu pur chez la fig. 7, est très incorrectement rendue.

Elle a été rencontrée par M. Dusés sur les troncs d'arbres à Bomana, à une altitude de 660 mètres.

\section{Perideris auripigmentum (REEVE).}

(Pl. V, Fig. 3, 4.)

1848 Bulimus auripigmentum ReEve Conch. icon. Vol. V. Pl. 29, N:0178.

\section{Orthalicus}

1856 Perideris
(Corona) Aisms H. et A. Genera of rec. Moll. II. p. 155.

Shuttleworta Notitix malac. p. 81. 
1859 Perideris auripigmentum Pferffer Mon. Hel. IV p. 595.

1860 Achatina $\gg$ (Perideris) Albers Heliceen ed. v. MAR* TENS p. 205.

\begin{tabular}{|c|c|c|c|}
\hline 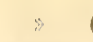 & (?) & Vignoni & Morelet Journ. de Conch. VIII p. 189. \\
\hline 1866 & $\gg$ & $\gg$ & $\begin{array}{l}\text { v. Martens Malak. Blätter XIII p. } 107 \text {, } \\
\text { T. 4, Fig. } 9 .\end{array}$ \\
\hline 1868 & 》Perideris & $\gg$ & Pfeiffer Mon. Hel. VI p. 204. \\
\hline 876 & » & auripigmen & m v. Martens Monatsber, Berlin p. 259. \\
\hline 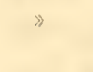 & > & $\gg$ & $\begin{array}{c}\text { Pfeiffer Novitates eoneh. IV p. 163, } \\
\text { T. 137, Fig. 5-7. }\end{array}$ \\
\hline & $\gg$ & $\gg$ & $\gg \quad$ Nomenclator p. 261 \\
\hline O & 》 & 》 & $\begin{array}{c}\text { KовеLt in Chemn. ed. Ков. p. } 37, \\
11 \text {, Fig. } 1-4 \text { (T. } 14 \text {, Fig. } 6 \text { ?). }\end{array}$ \\
\hline
\end{tabular}

Kaméroun: Itoki (S.); (Victoria (B.)).

(Calabar.)

\section{4 spécimens.}

Les quatre exemplaires que nous arous devant nous appartienment probablement à cette espèce, quoiqu'ils s'en écartent à divers égards, à en juger par la description donnée et par les dessins. Ils se rapprochent le plus de l'exemplaire reproduit par M. Ko BELT (1. c. T. 11, Fig. 1, 2), mais manquent totalement de la bande claire périphérique que l'on retrouve aussi chez les figures de Renve et de Presffer, et qui derrait par conséquent être caractéristique pour cette espèce.

Le dessin est plus régulier et se compose de flammes alteruantes, plus ou moins ondulées, d'un blane intense et d'un brun châtain presque noir, qui se dissolvent parfois, vers l'ouverture, en taches sur un fond couleur de chair passant au violet. (es flammes sont disposées de manière qu'une flamme foncée a toujour immédiatement à sa ganche mue tache blanche. mais jamais linverse. Les flammes foncées doivent être considérées comme des ramifications de la bande basale du même ton qui se trouve sous l'angle fortement accusé de la périphérie. Chez nos exemplaires. rette bande est jresque totalement continue. les stries blanches n'y descendant que peu ou point sous l'angle. Deux seulement montrent vers liouverture une couple de taches blanches. Jes flammes blanches sont opayues, et tant celles-ci que les flammes foncées oftrent presipue la même éridence dans l'interienr de la connille, qui y est reconverte d'me mince callosité blanc violet. 
Chez tous les exemplaires, on voit, vers l'ouverture, sur le dernier tour, les restes d'un épiderme jaune verdâtre. La columelle, bordée d'un callus blanc et entourée d'une étroite bande foncée, est, chez nos denx exemplaires adultes, presque absolument droite, légèrement oblique, et très faiblement tordue. Elle diverge par suite notablement de celle de la figure donnée par M. REeve.

Chez les exemplaires que nous avons entre les mains, il est impossible de distinguer de bord au-dessus de la suture entre les denx derniers tours de spire, par la raison que l'angle du dernier tour coïncide complètement avec la suture.

Outre les deux exemplaires assez courts et ventrus reproduits, nous en possédons un d'une forme plus étirée, avec le dernier toux plus aplati vers le milieu.

Chez tous les exemplaires, les bords du péristome sont reliés d'une façon parfaitement évidente par un mince callus qui pénètre quelque peu sous l'insertion du bord extérieur; suivant MI. KOBELT, ce callus n'existe pas chez les exemplaires qu'il a examinés.

Perideris Vignoni (MoRelet) n'est en général regardée que comme une forme juvénile de $P$. auripigmentum, mais nous osons demander s'il n'y aurait pas lieu de le considérer comme en constituant au moins une variété. Les deux exemplaires non adultes que nous avons sous les yeux se différencient du moins considérablement de l'espèce en question, et ressemblent en tout aux exemplaires adultes, avec la bande basale caractéristique, la région columellaire claire qui la suit immédiatement, et le plus près de la columelle, une étroite bande foncée, tandis que la figure de Perideris Vignoni donnée par M. v. Martens s'en écarte totalement à cet égard.

\section{Genus Pseudachatina AlBERs.}

Un trait fort caractéristique pour tout le genre Pseudachatina est la sculpture si particulière de l'apex que nous avons reproduite chez la figure $3, \mathrm{Pl}$. IV, représentant les tours de spire embryonnaires de Pseudachatina Martensi m. sous un triple grossissement. Comme on le voit, cette sculpture embryonnaire se distingue de celle du genre Achatina (voir 
Pl. Ill. Fig. 4), en ce que le nucleus même montre une fine sculpture spirale. qui passe en snite à me sonlpture presine "ompletement pareille à relle d'Achatina. mais pent-être un peu plus régulière.

()utre rette sculpture générique des tours embryonnaires, on aperçoit aussi. chez toutes les espèces du genre que nous arons en l'oreasion d'étudier, une striation en spirale très serrée et excessivement tine, visible seulement sous la loupe et à une lumière favorable. Cette sculpture se fait voir surtont sur les tours de spire inférieurs et particulierement sur les points dn dernier tour qui, quoique dépouillés de l'épiderme, nout pas été exposés à l'nsure. Elle fournit me constatation ultérieure de l'afhinité du genre Psendachatina aver celui de Perideris, chez lerpuel elle est néanmoins beancoup plus accusée.

En jugeant par l'apparence de la coquille. M. Kobera suppose que les espèces de ce genre habitent le sol, mais ce n'est mullement le cas; elles rivent par contre sur l'aherbe d'elephant: a larges fenilles, ainsi que sur le fenillage des arbustes at des arbres. ou on les troure fréquemment jusqu'a une hauteur de 5 metres. Comme Achatina marginata Swassox elles déposent leurs oenf's sur les arbres dans les angles des branches tout près du trone.

Tianimal est unicolore, grisâtre ou noiratre. et ressemble à celui des Achatina. mais les faihles cretes latérales du dos du pied que lim aperoit chez Achatina marginata ne se retrouvent pas chez les Pseudachatina.

\section{Pseudachatina Downesii (GRAY).}

$18+0$ Bulinus Downesii Gray in Sowerby Conchol. Illustr. Bulinus Fig. 99 .

1841 Achatina $\gg$ Pekiffen Symbola I p. 46.

1848 Bulimus $\gg$ Mon. Hel. II p. 15 (excl. syn. Grateloup).

1855 Preudachatina - Adas H. et A. Gen. of rec. Moll. II. p. 134, Pl. 75, Fig. 1.

1856 》 $\gg$ Shutrueworti Notitix malac. p. 85, 'T.

9, Fig. 3, 4 (excl. syn. Grateloup, Reeve, Albers). 
BIHANG TILL K. ST. VET,-AKAD. HANDL. BAND 22. AFD. IV. N:0 2. 87

1881 Pseudachatina Downesii Pferffer Nomenclator p. 266 (ex parte). 1893 ॠ $\gg$ Kent in Chemn. ed. Kов. p. 13 (excl. syu. Grateloup, Reeve, Albers, v. Martans) (nec figuræ).

Kaméroun: Isowi (J.).

17 spécimens.

On voit par la synonymie qui précède, que concernant cette espèce notre manière de voir diffère de celle qui a eu cours jusqu'ici. La grande quantité d'exemplaires des espèces du genre Psendachatina dont nous disposons, nous a mis dans l'impossibilité d'admettre comme appartenant réellement à Pseudachatina Downesii (GRAY) les nombreuses formes différentes qui ont figuré jusqu'ici sous ce nom. La raison des opinions divergentes qui se sont produites sur l'espèce en question, doit être attribuée, selon nous, da la circonstance que la figure donnee par Sowerby (l. c.) de Psendachatina (Bulinus) Downesii (GRIY), est peu satisfaisante, on aussi représente un exemplaire qui, tant de forme que de dessin, est assez peu commun pour l'espèce. Plusieurs des conchyliologistes qui l'ont décrite et reproduite après lui, y ont rapporté des espèces qui, selon nous, ne peuvent en aucune façon être considérées y appartenir. La petit nombre d'exemplaires des diverses espèces que l'on a pu jusqu'ici examiner, n’a naturellement contribué qu'à apporter iles difficultés à une notion claire de l'espèce. Ainsi, p. ex., les deux figures données par REErE ne nous paraissent pas y appartenir, mais d'être des espèces distinctes de relle-ci aussi bien qu'entre elles. Même les figures données par MI. Grateloup (Achatina Leaiana Actes Bordeaux Pl. 2. Fig. 7) et par M. r. Martens (Monatsber. Berlin April 1876 T. 2 Fig. 3) représentent d'autres espèces que l'on retrouvera dans les pages qui suivent. Nous n'avons pas non plus cité dans la synonymie qui précède les figures domnées par M. Kobelt sur cette espéce, la forme (l. c. T. 5, Fig. 1, 2) qu'il trouve concorder le mienx aree la figure originale de SowERBr, appartenant pent-être avec plus de raison a Ps. Martensi m., et nous renvoyons à cette espèce pour justifier notre opinion. M. Kóobet n'a donc pas en, selon nous, à sa disposition la vraie Pseudachatina Downesii (GraY). C'elui qui, à notre avis, a le mieux rendu 
l'espèce tant par la description que par la figure, est Snutruewortu (l. c.). Il paraît d'autant plus étrange qu'il ait conservé comme synonymes les formes de Grateloup et de ReEve, quoiqu'il exprime, il est rrai, ses dontes sur la question de savoir si les deux figures de ReEve penvent être considérées comme appartenant à la même espèce.

Dans son excellente monographie du genre Pseudachatina, M. Kobelt a rangé plusieurs variétés sous Ps. Downesii comme espèce principale. Celle de ces variétés qui est représentée parmi nos matériaux, savoir la var. Sodeni, doit, à notre avis, être élevée à la dignité d'espèce. Nous croyons, par contre, devoix ranger comme variété sous Ps. Downesii, Pseudachatina grandinata Pfenffer, que M. Kobert a maintenue comme espèce, tout en reconnaissant la faible différence qui la sépare de Ps. Downesii. M. Kobent déclare toutefois lui-même que son traitement du genre Pseudachatina ne doit être considéré que comme provisoire, et promet d'y revenir quand il aura des matériaux plus riches à sa disposition. Nous rerrions par conséquent avec plaisir que notre modeste contribution eût le mérite de jeter une plus grande clarté sur ce genre particulièrement difficile, même dans les cas où l'éminent savant ne pourrait pas partager en tout notre manière de voir.

Nous avons sous les yeux 17 exemplaires qui correspondent en tout à la diagnose de Snutruewortr. Che\% tous, le péristome et la columelle ainsi que le callus pariétal sont d'une couleur de chair claire, avec la région columellaire entourée d'une zone brun foncé n'atteignant pas l'angle du dernier tour de spire, mais se poursuivant sur la paroi de l'onverture assez loin sous l'insertion du bord extérieur. Cette zone est continue, plus ou moins nettement limitée à sa partie supérieure, et chez aucun exemplaire ne se dissout en flammes comme chez. Ps. Sorleni Kobelt. Les flammes d'm brun foncé qu'offre la figure de Sowerbi, manquent totalement chez tous nos exemplaires, et dans la diagnose de Shuttueworth elles ne sont pas non plus mentionnées.

A l'exception des tours de spire supérieur's, toute la coquille est converte de plis grossiers, simulant des cordons, s'étendant soit sous une forme plus ou moins anguleuse de suture en suture, soit interrompus ca et là, se bifurquant on se dissolvant en petits tubercules, mais formant sur d'autres points des callo- 
sités ou des bourrelets plus gros. Immédiatement au-dessus de la suture, ces cordons offrent un développement et un renflement plus forts, en aboutissant à une série dense de petites nodosités se continuant sur l'angle du dernier tour, pour s'affaiblir peu à peu ou même disparaître totalement, comme le reste de la sculpture, vers l'ouverture. Sous l'angle de la périphérie, la coquille est à peu près lisse à tous les âges.

La suture est linéaire, crénelée et bordée au-dessous d'un évidement dans les tours de spire plats au reste. La longueur varie entre 68 et $97 \mathrm{mill}$.

\section{Pseudachatina Downesii var. grandinata (PFEIfFer).}

(Pl. IV, Fig. 4, 5.)

1856 Pseudachatina grandinata Pfeiffer Malak. Blätter III p. 257.

1859

1881

1893
Mon. Hel. IV p. 598.

Nomenclator p. 267.

Koвelt in Chemn. ed. Koв. p. 23, T. 12, Fig. 2.

Kaméroun: Itoki (S.).

(Gabon (W ALKER).)

Un seul spécimen se trouve au Musée d'hist. nat. à Stockholm.

Nous ne croyons pas qu'il soit possible de conserver Pseudachatina grandinata Pfeiffer comme espèce, mais senlement comme une variété de Ps. Downesii (GraY). La seule chose qui pût constituer une différence spécifique d'avec la dernière, est la sculpture, qui chez Ps. grandinata doit se composer d'une granulation grossière et irrégulière, tandis qu'elle présente chez Ps. Downesii une apparence plus plissée ou cordée. Or, l'exemplaire que nous avons sous les yeux, et que nous considérons, malgré les différences de peu de valeur mentionnées ci-après, être Ps. grandinata Pfecffer, a par places ces cordons complètement dissous en une granulation grossière, circonstance que l'on retrouve aussi chez une partie des exemplaires de Ps. Downesii. Si l'on considère en outre que l'espèce de Pfiriffer est basée sur un seul 
exemplaire, il ne pent guere être possible d'admettre que cette sculpture suffise à constituer a elle seule une difference spécifique.

Nous croyons par contre que l'ouverture si petite en comparaison de la longueur du test, ainsi que la coulenr d'um blane pur du péristome, de la columelle et du callus pariétal, caractires par rapport anxquels notre exemplaire concorde exactement arec le type de M. Pferfor, foumissent une raison suftisante pour la placer comme variété sons Ps. Downesii (GRAY), qui. comme nous la eomprenons, doit tonjours aroir, a l'état adulte, ces parties colorées d'm chair elair on rouge de brique.

La variété se distingue en outre par une sculpture un pen plus grossiere que celle de l'espèce type. On retronve aussi chez elle, immédiatement au-dessus de la suture, la mème série de petites nodosités. Le dernier tour est distinctement anguleux, et l'impression au-dessous de la suture des tours, plats an reste, lui donne une apparence bi-anguleuse.

Le sommet est d'une couleur de chair très vive et sous l'épilerme d'un brun jaunâtre le reste du test est d'une teinte lilas pâle devenant sur le dernier tour presque purement blanche. La couleur châtain foncé de la base du dernier tour ne s'étend pas. à beancoup pris. aussi haut que sur la figure que M. Konelt en a donnée d'après un dessin original de M. Edaar A. Summ, ou elle se poursuit jusqu a l'angle du depnier tour. A l'exception de quelques traces de flammes conrtes sur la partie inférienre du quatrième tour de la spire. la coquille manque au surplus totalement de dessin.

\section{Pseudachatina Sodeni Kobelt.}

(Pl. IV, Fig. 6).

1848 Bulimus Downesii ReEve Conch. icon. Vol. V. P1. 29 N:o 177 a. 1876 Pseudachatina $》$ v. Marrens Monatsber., Berlin p. 259, T. 2, Fig. 3 (exel. syn. Gray, Pfelffer, SHUTtLew.).

1893

KoBEst in Chemn. ed. Ков. p. 16; T. 5 ,

Fig. :3-6; T. 8, Fig. 1; T. A, Fig. 1.

Kaméroun: Etome, Batoki, Basse (D.). 


\section{2 spécimens, dont 2 conservés dans l'alcool.}

Il est diflicile de roir dans cette espéce une variété de Pseudachatina Downesii (GRAr), vu qu'elle offre des différences beaucoup considérables.

La sculpture consiste, chez le deux espèces, en une costulation ressemblant i des cordons et anguleuse sur quelque, points. Mais chez cette espèce-ci, la costulation est sensiblement plus grossière et plus espacée, et présente des renflements et des noeuds beaucoup plus saillants. L'épiderme est aussi plus grossier et d'un brun verdâtre. La forme et la couleur basale de la coquille fournissent toutefois les principales différences spécifiques.

La coquille de Ps. Sodeni est toujours ovoïde, plus on moins allongée. avec le dernier tour de spire ventru et grand, comprenant, mesuré par derrière, à peu près la demi-hanteur de la coquille, tandis que Ps. Downesii a la coquille étirée en longueur arec les lignes marginales de la spire presque droites, ainsi que le dernier tour non ventru, et sensiblement plus petit par rapport à la longueur totale. Il en résulte que l'ourerture est chez la première infiniment plus grande que chez la seconde, circonstance qu'augmente encore le péristome dilaté en forme de cloche et fortement élargi che\% Ps. Sorleni. Il est plus ou moins épaissi, et ainsi que la columelle et le callus pariétal, d'un rouge de brique clair chez les exemplaires adultes; environ an milien de la paroi, ce callus s'amincit subitement comme chez toutes les espèces du genre, et montre la couleur du fond par transparence. M. Kobelt signale, comme un trait caractéristique chez Ps. Downesii et les espèces voisines, la circonstance que ce callus porte en haut une bande foncée. C'ela dépend pent-être d'ume méprise ou pourrait y donner lieu, cette bande foncée n'appartenant nullement au callus, mais, comme il a été dit plus haut, constituant la couleur basale foncée qui naturellement se continue sur la paroi, et y est visible par transparence autour de la partie opaque du callus.

La région basale manque de la zone foncée, continue, plus ou moins nettement linitée, qui distingue Ps. Downesii et plusieurs antres espèces du genre, et qui est an lieu remplacée ici par de larges flammes brunes conleur châtain, ne confluant pas en bas, mais rayonnant en haut depuis la co- 
lnmelle, et s'étendant jusqu'au voisinage et même au-dessus de l'angle périphérique d'ordinaire très faible et toujours moins accusé que chez Ps. Downesii.

La longueur de la coquille varie entre 76 et $105 \mathrm{mill}$.

Pseudachatina Dennisoni Pfeiffel et var. connectens $m$. (Pl. IV, Fig. 1-3.)

1838 ? Achatina Leaiana Grateloup in Actes Bordeaux p. 416, Pl. 2, Fig. 7.

1848 Bulimus Downesii var. Reeve Couch. icon. Pl. 29, N:o 177 b. 1856 Psendachatina Dennisoni Pfelffer Malak. Blätt. III p. 257.

Kamérom: Bomana (D.); Itoki, Bonge. Bibundi (S.); (Grabon). Isowi (J.).

51 spécimens, dont 8 conservés dans l'alcool.

Quoique les exemplaires que nous avons devant nous ne concordent pas completement, à quelques égards peu essentiels du reste, arec la diagnose de Preiffer, nous croyons néanmoins ponvoir, d'après la figure de ReEve, les rapporter à l'espèce mentionnée ci-dessus.

Ils forment au surplus une série dont l'un des points terminaux est en proche conformite de cette tigure, tandis que le second point se rapproche davantage de Psendachatina Sorleni Konet, Ils ont tous, en analogie aver la figure de REEve l'apex et les tours de spire supérieurs plus on moins profondément rouleur de chair, tandis que la diagnose de Pfetreer indique un apex noiritre; la columelle, couleur de chair dans la tigure de REErE, offre, chez tons nos exemplaires, le blane pur yu'indique Pfeiffer. Le péristome est, chez la plupart. d'me conleur de chair on rosée. Quelques-mus seulement l'ont blanc, avec la partie inférieure d'une nuance violette assez foncée, due a la couleur sombre de la zone basale translucide. Le reste de la coquille est d'un rose plus on moins fonce, parfois d'un brun pur, parfois aussi presque b]anc. 
Dans la plupart des cas, la suture est marginée par une ligne imprimée, quoiqu'elle soit qualifiée de non marginata chez Pfeiffer. Les exemplaires qui se rapprochent le plus de la figure de ReEve, ont le dernier tour arrondi, sans trace d'un angle qui, par contre, s'accuse très fortement vers le second point terminal de la série. Nous avons reproduit l'exemplaire fermant la série de ce côté (Pl. IV, Fig. 1).

La base de la coquille porte chez tous les exemplaires une zone brun châtain foncé à bord supérieur plus on moins nettement tranché, qui n'atteint que très rarement l'insertion du bord extérieur de l'ouverture, et qui n'est par conséquent jamais visible, comme chez le type de ReEve, sous la forme d'une bande foncée au-dessus de la suture. Chez la jeune. coquille, cette zone s'étend par contre fréquemment en flammes régulières radiantes, dont la partie considérablement plus claire située au-dessus de l'angle, donne naissance dans ce cas aux flammes triangulaires que l'on rencontre souvent sur les tours de spire supérieurs, et qui ne s'étendent que chez un seul exemplaire jusqu'à l'avant-dernier tour.

La sculpture qui, chez les exemplaires les plus typiques, ne se compose que d'une costulation grossière parfois légèrement arquée, dissoute çà et là en tubercules, on en arêtes saillantes à dos d'un blane de craie, reçoit, chez le groupe représenté par notre figure, une apparence qui, quoique plus régulière, ressemble beaucoup à celle de Ps. Sodeni. On ne retrouve cette conleur blanc de craie chez aucune des espèces que nous avons eu l'oceasion d'examiner du genre en question. Chez Ps. Sodeni, qui s'en rapproche également ici le plus, elle n'est pas plus claire que les parties les plus claires du fond, qui ne sont jamais d'un blanc de craie.

L'apparence étagée des tours de spire, qui est si prononcée dans la figure de REEvE, se retrouve à un degré remarquable aussi bien chez l'exemplaire reproduit par nous que chez plusieurs des exemplaires que nous avons entre les mains.

Le péristome est plus ou moins dilaté, mais moins en général que celui de Ps. Sodeni.

La forme que nous avons reproduite, mériterait probablement d'être séparée comme variété, quoiqu'elle paraisse se relier par des transitions au type de ReEve. Nous serions disposé dans ce cas à proposer pour cette forme le nom de Pseudachatina Dennisoni var. connectens. 
Le manque d'exemplaires typiques de Ps. Gabonensis SHutrueworth nous met dans l'impossibilité de determiner les relations de rette espece avec Ps. Dennisoni. La diagnose de l'éminent conchyliologiste paraît dénoter des relations très rapprochees. Mais cet illustre naturaliste déclarant que le péristome n'est jamais épaissi. que la columelle n'est que faiblement tronquée, et les figures 1- ̈ données chez FÉrussac (Hist. nat. II, Pl. 122) et par Il. Kíoeelt (1. c. T. 9, Fig. 1-6) paraissant représenter une espèce bien distincte de Ps. Dennisoni Premfrer, il n'est guère facile de les réunir. „uoique loriginal des figures 5 et 6 reproduites par II. KoneLt se trourat sons le nom de Ps. Demnisoni dans la collection P.eteu.

Nous avons cité, aver un point d’interrogation, dans la symonymie qui précède, l'Achatina Leaiana de Gratelocp. considérée jusqüiei par les auteurs comme synonyme de Psendachatina Downesii Gray. ()r, de la manière dont nous comprenons cette dernière espèce, nous ne pourons pas adopter cette manière de voir. La figure donnée de M. Gratelonp concorde, dans la forme, presque entièrement avec Ps. Gravenrenthi, mais la description de la sculpture et de la conleur du test défend de l'y rapporter.

Pseudachatina Gravenreuthi Bötritien et var. Preussi Konflr.

1891 Psendachatina Gravenreuthi Börtager (fide Staudinger). 1893 KOBELT in Chemn. ed. Kon. p. 18, T. 6, Fig. 3, 4; T. 7, Fig. $1-4$.

Kaméroun: Etome (D.); Buea (à 950 m. d'alt.: D.; J., Рв.).

Pseudachatina Gravenreuthi var. prenssi KobelT ibidem p. 21, T. 6. Fig. 5. 6; T. 7. Fig. 5, (i.

Etome (D.); (Buea (Pr.)).

$\checkmark$ spécimens de la forme typique. 2 spéc. de la variéte. tous recueillis à l'état vivant.

(Quatre exemplaires appartenant à la forme principale sont d'une conleur châtain très toncé, qui ne se retrouve

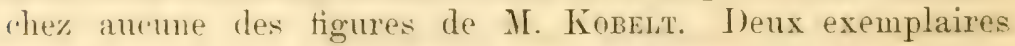


paraissent par leur taille mince et leur teinte sombre appartenir à la variété preussi KoßELT, tout en étant cependant un peu plus longs.

A en juger par des individus conservés dans l'alcool, l'animal est d'un brun noirâtre, unicolore.

\section{Pseudachatina Martensi n. sp.}

(Pl. IV, Fig. 7-9.)

T'esta imperforata, ovato-oblonga vel turrita, solida, crebre et sat regulariter plicato-striata, sub epidermide squamosa, in anfractibus superis plerumque decidua, in ultimo ad aperturam siepius persistente, nitida, albida vel roseo-carnea apice saturatiore, rarissime strigis rufis, pancis, brevibus, evanescentibus, in anfractibus medianis supra suturam obsolete ornata, basi atro-castanea. Spira convexo-conica vel turrita, apice obtuso. Anfractus $7^{1}: 2$, primi 4 convexinsculi, lieves; sequentes plus minusve planati, infra suturam anguste marginatam impressi, irregulariter grosse et remote cristato-plicati vel rarius tuberculis conicis. distantibus supra suturam sat regulariter instructi; ultimus postice $1 / 2$ longitudinis fere iequans, plus minusve distincte angulatus, angulo aperturam versus evanescente. Apertura obliqua, rotundato-ovalis, intus albida vel albido-carnea, leviter margaritacea, zona fusca basali externa subtus translucente; columella compressa, intorta, peroblique truncata, alba; peristoma anguste reflexum vel reflexo-patulum, modice limbato-incrassatum, semper album, marginibus callo albido, columellam versus incrassatoopaco, insertionem marginis dextri versus diaphano, junctis.

Long. 47²-75 mill.; diam. 25-33; apert. alt. 20-31 mill. (cum peristomate).

Habitat in Camerunia: Etome (D.), Itoki (D., S.).

Specimina 68, quorum 18 in alcoh. servata.

Malgré une étude détaillée de tontes les descriptions et de tous les dessins de ce genre difficile, il nous est impossible d'identifier l'espèce décrite ci-dessus avec une espèce déjà connue. 
Par son habitus général et par le système de coloration. elle rappelle légèrement Pseudachatina Wrigthi var. Buchneri Kobelt (Chemn. ed. Ков. p. 11; T. 4, Fig. 5, 6), mais offre toutefois des caractères interdisant de l'y rapporter. La zone basale châtain foncé, parfois presque noirâtre, n'atteint jamais l'angle dn dernier tour, mais pénetre dans la paroi de l'ouverture sous l'insertion du bord extérieur, et est visible dans l'ouverture en dehor's de la partie du callus pariétal devenue opaque par épaississement. Vers le haut, cette zone est très nettement limitée, beaucoup plus nettement que chez aucune autre espèce, et présente le même aspect à tous les âges de la coquille (voir $\mathrm{Pl}$. IV, Fig. 9, représentant un exemplaire non adulte avec 6 tours de spire et de 34 mill. de longueur, vu de la base).

Chez les individus adultes, la coquille est solide et épaisse; chez les jeunes elle est mince et demi-transparente. La coquille de l'embryon est munie d'une perforation évidente, qui se maintient chez la jeune coquille jusqu'à ce qu'elle ait atteint environ $4^{1 / 2}$ à 5 tours de spire, en même qu'elle fait voir une liste calleuse très nette, limitant le côté interne de la columelle et en indiquant la forme future.

A l'exception des tours de spire embryomnaires, qui présentent la sculpture commune à tout le genre, la coquille est munie d'une striure de croissance assez régulière et a plissures très fines, qui est plus fortement accusée dans les tours de spire médians. A côté de cette sculpture se présente, à partir du $5^{\text {me }}$ tour depnis lapex, une autre sculpture de nature toute différente. Celle-ci se compose de gros renflements irréguliers, formant des plis ou des crètes grossières. Ils sont le mieux caractérisés chez les exemplaires a coquille ventrue, moins chez les turriculés (Cf. Pl. IV, Fig. 7 et s). Cette sculpture est moins accusée sur le dernier tour, et dans la righle elle disparait totalement vers l'ouverture. Elle se présente chez quelques exemplaires sous la forme de bosses pointues placées à égale distance les unes des autres sur l'angle basal des tours de spire, et par conséquent visibles immediatement au-dessus de la suture. La figure 9 donne quelques indications it cesujet. Chez un seul exemplaire, cette dernière sculpture manque presque totalement, le test ne présentant que la fine sculpture d'accroissement. Or grice is cette eirconstance, l'exemplaire en question oftre une ressemblance 
frappante aree les tigures qua clomees M. Kombut de Pseurlachatina Downesii (Grar) (1. c. T. 5), Fig. 1, 2). Selon M. Kobelt. cette forme concorde le mieux avec la figure originale de $\mathbf{M}$. Soweruy pour Ps. Downesii, mais a en juger par les figures. nous ne pourons pas partager son avis sur ce sujet. Elles appartiennent peut-être arec plus de raison à notre espèce. Comme le font voir nos fig. 7 et 8 , la forme de la coquille, avec la forme d'ouverture en résultant, est très variable, et l'exemplaire en question est encore plus turriculé que celui reprodnit par la tig. $s$, circonstance par suite de laquelle la ressemblance arec les figures de M. Kobelí devient encore plus considérable.

La couleur est ou d'un blanc pur ou d'une teinte rosée augmentant d'intensité vers l'apex, qui, dans la plupart des cas, est couleur de chair. Chez un seul exemplaire, qui est à part cela d'un lilas clair, il est toutefois d'un noir riolet. et chez les exemplaires diun hlanc uniforme, il est d'ordinaire d'un blane pur. La partie supérieure des tours de spire au voisinage immédiat de la suture est plus claire chez les exemplaires roses. Au reste, tous nos exemplaires adultes manquent de toute trace d'un autre dessin que la zone basale foncée. Par contre, chez les exemplaires non adultes avec 4 à 5 touxs de spire, cenx qui suivent immédiatement les tours embryonnaires portent quelques flammes d'un hrun clair montant de la suture, mais ne dépassant pas dans leur partie inférieure l'angle périphérique fortement accusé chez les jeunes individus, et par suite ne formant pas continuiti avec la zone basale.

Quelle que soit la conleur fondamentale de la coruille. le péristome est, de mème que la columelle, toujours recouvert d'mn callus d'un blane pur, qui ne recoit qu'en bas une teinte plus foncée par suite de la zone basale translucide. Le dernier tour de spire est plus on moins évidemment angulenx; quelques exemplaires ont même conservi a l'état adulte la torme de la jeunesse, qui montre toujours un angle fortement marqué.

L'espèce offre en outre dans la règle, sur les trois derniers tours de spire, quelques sillons irréguliers et inter. rompus, que l'on rencontre aussi chez Ps. Buchholzi Konelt. dont M. le Professeur v. Martens a eu la bonté de nous prêter un type, et qui constitue, selon nous, une bonne espèce, 
ainsi yue chez Ps. Tiljevalli m., mais qui paraissent manquer totalement chez les autres espèces du genre à nous commues.

La suture est etroitement marginée et an-dessous d'elle les tours de spire présentent un évidement plus ou moins mal'pué.

L'epiderme, qui reste en place le plus surrent senlement sur le dernier tour vers l'ouverture, a l'apparence fenillée ordinaire, et la couleur en est jaune brun.

La grandem de la coquille est très variable: nous avons p. ex. des exemplaires de $47^{1} / 2$ à 75 mill. de longueur.

\section{Pseudachatina Liljevalli n. sp.}

(Pl. V, Fig. 1, 2.)

Testa imperforata ovato-(0)nica, tenuis sed solida, leviter plicato-striata sub epidermide sumamosa in anfractibus superis decidua in ultimo ad aperturam persistente. nitida, cameoracatica, anfractibus superis atro-purpureis. strigis subtilihus superticialihuspue alhido-corulescentibus pictis, anfractibus medianis interdum strigis fuscis. latis, et albis angustiorilus interjectis ornatis, anfractibus 3 ultimis infra sutmram ticnia lata allida circumcinctis, nltimo basi zona atro-cacaotica, insertionem marginis dextri attingente, cincto; spira convexoconica, apice olituso, sculptura embryonali propria et peculiaria generis ornato. Anfractus 7, sutura anguste linea impressa marginata disereti. primi t convexiusculi, subleves, sepuentes planati, infra suturam impressi. plus minusve regulariter rotundato-cristato-plinati. plicis pristrim sursum infra marginationem suturie prominentibus; ultims postice ${ }^{1}:$ longitudinis tere ayuans, distincte angulatus, angulo prope aperturam evamescente. Apertura oliliqua, subrotundata, intus tricolor, antractu ultimo utringue concolore; columella valde intorta, oblinur truncata, alba: peristoma anguste reflexum, callo alhido-limbatum, marginibus eallo alhido, columellam versus incrassato, opaco, insertionem maroginis dextri versus diaphano, junctis.

Long. 45-501/2 mill.; diam. 24-27; apert. alt. 20-22 mill. (cum peristomate).

Habitat in Camerunia: N'dian (S.). 
Specimina 2, unum in alcoh. servatum.

Cette petite espèce ne peut être assimilée à aucune de celles jusqu'ici connues, et doit être considérée comme une espèce nouvelle parfaitement distincte.

Elle se fait remarquer en premier lien par sa taille diminutive, l'un des exemplaires mesurant 5()$^{1} 2$ mill., et le second 45 mill. seulement de longueur.

La coquille assez mince, quoique solide, est de forme ronique ovoïde, avec tours de spire fortement imprimés sous la suture marginée par une ligne profonde et dès lous disposés en étage. Sous cette ligne, les tours de spire sont munis de grosses callosités assez régulières, sensiblement plus fortes vers la suture, et qui, commençant au $4^{\text {me }}$ tour à compter d'en haut, disparaissent presque totalement au dernier tour vers l'ouverture. A l'exception de l'apex faisant voir la sculpture embryonnaire rommune an genre entier, la coyuille est munie d'une sculpture de croissance irrégulière assez fine et striée en plis. Les fines stries spirales que nous avons eu l'occasion de mentionner en parlant des caractères communs à tout le genre Pseudachatina, sobservent chez cette espéce-ci plus haut vers l'apex que chez aucune les autres espèces mentionnées. On peut voir une sculpture secondaire dans les sillons en spirale déprimés et interrompus des denx derniers tours de spire, en analogie avec cenx yue l'on rencontre chez Ps. Martensi m. et Ps. Buchluolzi KoвELT.

La base de la coquille jusqu'à l'angle fortement développé du dernier tour, ainsi que les quatre tours de spire supérieurs, sont d'une conleur chocolat foncée. Partout ailleurs la coquille est d'un chocolat rouge clair, a l'exception d'une large zone blanche immédiatement au-dessous de la suture. Chez le grand exemplaire, la zone basale foncée est nettement limitée vers le haut, de même que la zone suturale blanche vers le bas. Par contre, chez le petit exemplaire reproduit, la zone basale envoie en hant des flammes foncées. séparées par les flammes blanches partant de la zone suturale. qui suivent surtout le dos des arêtes renflées. On remarque en outre chez les deux exemplaires, sur les troisieme et quatrième tours foncés, comptés d'en haut, de petites stries blancher rendues bleuâtres par la couleur foncée sous-jacente. 
L'intérieur' de l'ouverture montre les mêmes zones de couleur différente que l'extérieur du dernier tour.

Le piristome est itroitement refléchi, et le bord extérians. qui est muni en dedans d'une lèvre blanche, est blanc chez. lin des exemplaires, mais nuance chez l'antre en conformiti: de la coloration extérienre.

La columelle assez fortement tordue ot ividée est hlanche. ut les hords de lourerture sont reliés entre eux par un callus blane translucide vers l'inseution du bord externe. L'épiderme janne brum yui fresente l'apparence particulitue du genre. n'est conservé qu'au dernier tour vers l'ouverture.

Ce nous est un plaisir de dédier cette belle petite espèce ¿̀ M. Lituevalu, l'éminent dessimateur de nos figures.

\section{Gentts Pseudoglessula Büttger.}

\section{Pseudoglessula clavata (GraY) et rar. Grayi m.}

1837 Aehatina clavata GRAY in Charlesworth's Magazine, New Ser. Vol. 1. 1. 487.

1838 > WIEGM. Arch., II, p. 275.

1848

1849

1855

1865

1868

Subulina

Pfeiffer Mon. Hel. II p. 260.

1876 Stenogyra Calabarica v. Martens Monatsber. Berlin, p. 260, T. 3 , Fig. 5, 6 .

1881 Glessula clavata Pferferi Nomenclator p. 331.

\section{$\gg$ Calabarica » 》 》}

1892 Pseudoglessula calabarica BöTteger in Nachrichtsbl. malak. Gesellsch. p. 202.

1894 Homorus (Pseudoglessula) calabaricus Kobert in Chemn. ed.

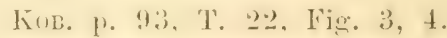

Kaméroun: Etome, Bomana (D.); N'dian, Bonge (S.); Isowi, Buea (J.); Bibundi (D., S. J.) (Bonjongo (B.)).

(Old Calabar.)

54 spécimens, dont 3̉o recueillis à l'état vivant.

La sculpture du dernier tour a été décrite comme suit far M. Linesta oberseits scharf gestreitt, die streifen an der 
Kielkante plützlich abhrechend, die Unterseite glatt. ()r. ce n'est nullement le cas: les stries se continuent au contraire sans interruption par la carène pour se perdre enfin, après nn léger affiblissement qui s'observe surtont chez les jemes individus, de l'antre côté de la carène, sous la callosité qui réunit les bords de l'ouverture, tout en étant même quelprefois perceptibles it travers. Selon l'illustre savant, l"intérieur de l'ouverture serait de la même conleur que la surface de la coquille, ce qui est aussi contraire à toutes les observations que nous avons faites sur les exemplaires ì notre disposition. Chez cenx-ci, l'ouverture est colorée à l'intérieur d'une nuance blanchatre tirant sur le blen, qui, che\% les individus recupillis morts et dérolorés. s'approche plus ou moins du blane. La callosité rémissant les bords de l'ouverture devient plus épaisse sur la columelle qui, comme chez toutes les P'seudoglessula, est bordee d'un blanc callenx. tout en étant an surplus tronquée transversalement chez les uns, obliquement chez d'autres.

En comparant l'Achatina clavata de GrAY avec l'A. Calabarica de Pferffer, nous ne samrions trouver un seul trait caractéristique d'une importance qui antorise a en fair. denx espèces, en tant qu'on puisse se fier anx diagnoses et aux figures qui en ont été données. Les différences que l'on peut trouver, se rapportent à des caractères d'une valeur très douteuse (du moins dans ce cas), et dont chaque con"hyliologiste a en des ocrasions imombrables de ronstater l'inconstance. Telles sont: la forme entière de la coquille, la coloration et l'échancrure de la columelle. Liimportance que l'on pourrait être à même d'attribuer dans ce cas aux diftérences de grandeur et de forme, résultera de ce qui suit.

Selon la diagnose, Achatina clavata Gray mesure 28 mill. de longueur sur 1:i mill. de dianètre (Fig. 1), tandis que la longueur d'A. Calabarica Pfeiffer est de 28 mill. sur 11 de diam. (Fig. 2); la première est done plus large sur la mème longueur, tandis que selon la finure qu'a domnée II. Reeve d'A. clavata, cette espèce, quoique plus longue (34 mill.) ne mesure que 10 mill, de diam. (Fig. 3), étant ainsi moins large qu'A. Calahariea. Cette variabilite dams les mesures d'A. clavata a déjà été observée par M. v.

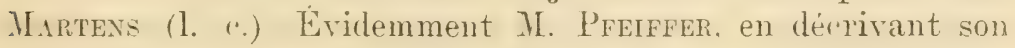
espèce, n'a en it sa disposition que de jemnes exemplaires; or 
nous arons devant nous non-senlement des spécimens qui. avec 10 tours et demi. mesurent 45 mill. de longuem sur 17 de diam. (Fig. 4), surpassant ainsi tous les individus dérit: et tigurés de rette espere. mais aussi des spécimens (11 expmplaires provenant d'm hois sombre et humide a Bonge), yui. aver la spire de 9 tours et demi. sont d'une longmeur de 24 mill. sur seulement \& de diam.; ayant un demi-tour de plus de développenent. cenx-ci répondraient partaitement a la figme de II. ReEve. Entre res deux extromes se voient en outre des formes de transition. A notre avis, il résulterait donc de ce qui vient d'être relaté, yue la forme le la corguille offre, dans ce cas, une raleur de peu d'importance.

Fig. 4 .

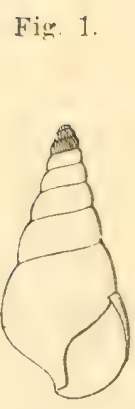

clavata .
Fig. :

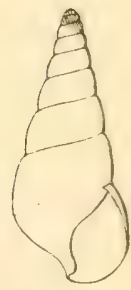

Calabarica.

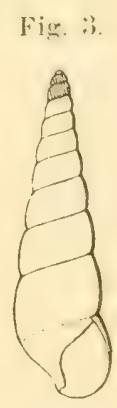

clavatia.

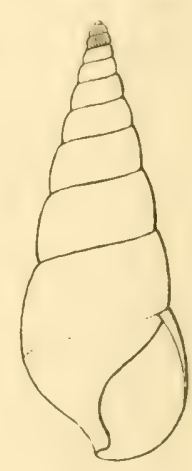

clavata

Pseudoglessula: Fig. 1 Achatina clavata GrAY selon la diagnose: fig. 2 Achatina Calabarica PremfFer; fig. 3 Achatina clavata GraY selon la fig. de ReEve; fig. 4 Psendoglessula clavata d'aprés un de nos exemplaires.

En parlant de la coloration d'A. clavata, MI. Pfenffer se sert de ces termes: sub epidernide cornea albida», et $\mathbf{M}$. REEve la déreit ainsi: albila. ppidermide sordide straminea induta ; $A$. "alabarica, an contraire, est caractérisée comme fusco-nigrieans interdum pallidius strigata. Nous avons pourtant entre les mains des indivilus d'une couleur brume. pálie par la décoloration de la conuille. ('omme en ontre nonseulement selon MII. PrEmFrik et KonibLt, mais aussi selon les observations faites sur plusieurs de nos sujets, qui ont ite recueillis frais. la conlemr fonce est quelquetois a et là 
plus ou moins pâle, il est permis de conchure que la nuance pâle peut devenir la prédominante, sans que pour cela il faille songer à des variétés albinos. Enfin, quant à la colımelle, elle est, comme il a été mentionné plus haut, le plus souvent, brusquement tranchée. quelquefois obliquement. Nous retrouverons l'inconstance de ce caractère chez Achatina marginata SwaInson, dont la columelle est aussi coupée dans les deux sens. La valeur de ce caractère comme signe de distinetion entre les espèces est donc très problématique. La diffèrence chez A. clavata entre la sculpture de l'apex et celle du reste du test est positivement indiquée par MI. Grar, et on la voit aussi dans la figure, ce qui ne laisse aucun doute sur la place de cette espèce parmi les Pseudoglessulie.

La carène du dernier tour, quoique non mentionnée dans la diagnose d'A. clarata, est cependant désignée sur la figure représentant l'espèce.

Enfin, n'est-il pas singulier que l'espèce de M. Grar n'a pas été retrouvée sur les lieux indiqués, malgré le grand espace de temps qui s'est écoulé depuis sa découverte?

Voilà les raisons qui nous ont forcé de réunir ces deux espèces sous le nom de Pseudoglessula clavata (Gray). La forme svelte sur laquelle la diagnose de M. Gray a été fondée ayant pent-être néanmoins le droit d'être considérée comme une variété, nous proposerons pour elle le nom de Psendoglessula clavata var. Grayi.

Dans plusieurs individus, nous avons trouvé des embryons d'un nombre oscillant entre 8 et 18 , dont la spire compte environ trois tours et dont les dimensions varient de $3^{1}$ a a 5 mill. de longueur sur $2^{1 / 2}$ à 3 mill. de diam. Ils sont tous d'une couleur blanche tirant plus on moins sur le verdatre. c'est-ì-dire la conleur propre au test adulte sous l'épiderme. Chez les plus grands, le péristome est bordé d'un brun foncé, et tous ont le dernier tour fortement anguleux, avec la base à peu près lisse, gravée seulement de stries d'une finesse extraordinaire. La forme de la columelle ressemble exactement à celle des adultes. Liespèce est donc ovovivipare. 


\title{
Pseudoglessula Sjöstedti n. sp.
}

\author{
(Tah, Y, Fier. li, T.)
}

Testa oblongu-conica, turrita. solidiusula. plicato-striata. fusc(s)-olivacea, hiv illic strigis satmatioribus obsolete ornata. Spira apicem versus concava, apios ('ylindrico, obtusinseulo. Antr. (9) , convexiusuli, sutura superne filo-marginata discreti. Anfr. :) embryonales retioulato-foreolati, convexi; sepuentes irresulariter plirato-striati. nltimus: rirea 1 : longritudinis iеpuans, ad neripheriam filo-cinctus, antire haur descendens, subtus attemuatus. Colmmella cmvata, albo callo-marginata. nume sat abrupte, nunc oblique truncata. Apertura subvertivalis, sulstriangulari-ovata, intus coprulesente-margaritarea: freristoma simplex, arntum. tenue; marginibus callo tenuissimo junctis.

Long. 42 mill.; diam. $15^{1 / 2}$; apert. lat. $8^{1 / 2}$, alt. $14 \mathrm{mill}$.

Habitat in Camerunia: Bonge, Bibundi, Itoki (prope Hum. Massalie) (S.).

\section{Specimina 11 exstant, omnia viv. coll.}

Quoique infiniment voisine de Ps. heteracra BötTGER

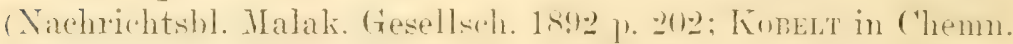
ed. Koв. p. 94 'T. 22 Fig. 5-8), cette espèce présente néanmoins des particularités qui nous empêchent de les considérer comme identiques. Le dernier tour de la spire de notre espèce, qui ne descend nullement vers l'ouverture, est toujours pourvu d'un angle obtus, filiforme, sous-bordé d'une exraration lisere et entrerouper par les stries pliciformes de la surface, qui se continuent à la base. On apercoit aussi cet angle sous la forme d'un til mespue papillenx marginant la suture, l'enroulement des tours ne coïncidant pas exactement avec lui. La columelle ressemble à celle de l'espèce

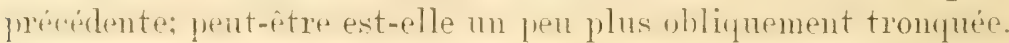
lionverture. dime teinte henatre à linterisur. varie plus on moins dans :a forme, dépendant de relle de la rolumelle, yui est plus ou moins courbée. La spire est beaucoup plus concave que chez les autres espèces de ce groupe; les tours moins convexes, séparés par une suture moins profonde, le dernier 
tour moins ventru vers la base que chez Ps. clavata. La surface est gravee d'une soulpture moins fortement acrusée, se composant de stries pliciformes assez espracies, plutot que costulées, comme chez la précédente. La sculpture singuliere ot élegante des tours embryonnaires nous semble tout a fait pareille à celle de Ps. heteracra. Chez tous nos exemplaires, dont le plus grand, comptant 9 tours de spire et demi, mesure 42 mill. de longueur, e'est-à-dire un tiers de plus qu'il n'a été assigné à Ps. heteracra, l'épiderme, d'un brun foncé olivitre et d'un éclat soyeux à l'état frais, manque partiellement sur la parri de l'ourerture, montrant le test d'une coulemr de paille ou de cire et d'un éclat assez brillant, trait que l'on retrouve chez presque toutes les especes appartenant is ce genre. Les oeufs, d'une forme allongée, caractéristique ponr re genre, d'environ is mill. de longuen sur à de diamétre, sont d'un blanc pur et d'nu éclat mat; ils ont dans l'oviducte la meime position oblinue que chez Ps. retifera (v. Martens) et Ps. involuta (Gould).

Cette espèce a été trouvée en 1891 avec Ps. retifera et clavata par M. SJöstent, anquel nous la dédions.

Comme ses congénères, elle vit parmi les feuilles mortes, et la plupart de nos exemplaires étaient, sur la moitié supérieure, couverts d'une croute de terre mince, mais d'une consistance très solide et aidhérente.

\section{Pseudoglessula retifera (V. Martens).}

1876 Stenogyra retifera v. Mar'tens Monatsber. Berlin, p. 260, T. 3, Fig. 7, 8.

1895 Homorus retifer (Psendoglessuli) KoBelt in Chemn. ed. KoB. p. 106: T. 30, Fig. 7, s.

Taméroun: Kumbe (D.); Bonge. Kitta, N'dian (S.): Bibundi (S., J.); (Bonjongo (B.)).

38 spécimens, dont 31 recueillis à l'état vivant.

La coquille en bon état est demi-transparente et d'un eclat soyeux. Le dernier tour n'est pas anguleux, il est vrai, mais il est néanmoins pourvu d'une carène aplatie et filiforme, dont M. V. Martess ne parle pas, et qui ne manque que rhez un seul individu qui endommage pendant sa rrois- 
silnce, a été ainsi contrarié dans son développement. Par la même cause, cet individu n'a pas le dessin carartéristique. In waratire que partage rette espere aren presine tontes les autres appartenant au même grompe, est la perte partielle de l'epiderme sur la paroi de l'ouverture, laissant ainsi roir la couleur propre du test. d'une conleur paille pâle tirant sur le verlatre, circonstance visible aussi dans la figure qu'en a donnée M. v. Martexs. La columelle est bordée d'un callus blanchâtre.

La description que donne de cette espèce l'éminent conchyliologiste allemand, se fonde sans doute sur des exemplaires jeunes, car nous en avons qui comptent 10 tours et mesurent 41 mill. de longueur sur 20 mill. de diamétre. l'onverture ayant 14 mill. de hauteur sur 7 et demi de largenr.

Les oeufs sont de la forme oblongue qui caractérise le groupe; ils mesurent 4 mill. sur $2^{2} / 3$, avec coloration d'un jaune pâle, blanchâtre. Ils sont rangés obliquement dans l'oviducte. comme chez Pseudoglessula involuta (GotLd), et l'individu le plus grand en contenait 10.

\section{Pseudoglessula fuscidula (Morelet).}

1858 Achatina fuscidula Morelet Séries conch. I p. 26. Pl. 1. Fig. 9. 1868 Pfeiffer Mon. Hel. YI p. 238.

1881 Stenogyra $>$ (Subulina) Preiffer Nomenclator p. 328.

Kaméroun: Ekundu-Etitti. Boangolo (I).). Bonge. Itoki (S.).

(Gabon.)

5) spécimens, dont 2 recueillis à l'état virant.

Tous les caractères de cette belle petite espèce lui assignent me place parmi les Psendoglessula. Les tour's amhryomaires sont ornés de côtes régulieres plus espacées entre elles (surtout sur les denx premiers) et moins obliques que celles du reste du test, ou elles deviennent irrégulieres et obliquement arquées; le dernier tour est pourvu d'un angle filiforme.

Sous l'épiderme, qui est très mince, sans éclat, et qui se détache aisément, spécialement sur la paroi de l'ouver- 
ture, comme chez les espèces précédentes, le test est brillant. d'une couleur de cire tirant sur le rerdâtre. et domnant, par transparence. un éclat mat d'une apparence soveuse à la coquille, avec addition d'une nuance de rert à sa couleur d'un brun çà et là un peu plus foncé. Les bords de l'ouverture sont réunis par un callus très mince, et la colunelle est marginée d'un blane calleux.

L'individu sur lequel M. Morelet a fondé la diagnose et dont il a donné une figure, n'a pas dû être adulte, le plus grand des exemplaires que nous avons examinés mesurant 11 mill. et demi de longueur sur: $:$ de diametre, avec ? tours et demi de spire.

Cette espece. vraisemblablement assez rare. a été rencontrée non-seulement sous des feuilles mortes, mais aussi sur des plantes. On l'y trouve une à une, jamais en abondance, comme les Subulinæ.

\section{Pseudoglessula Duséni n. sp.}

(Tab. V, Fig. 8-10.)

Testa oblongo-turrita, tenuis, fortiter filo-costata, sub epidermide rufo-fusca, apicem versus pallidiore, hic illic strigis saturatioribus obsolete picta et in parie aperturali facile deterenda, albido-virescens. Spira turrita apice cylindrico: obtusulo. Anfr. 101/2, convexi, leniter crescentes, sutura impressa discreti; embryonales. vertice ipso mammillato levigato excepto, subperpendiculariter regulariterque costulati, costulis in tertio sapius subtus evanescentibus; serpuentes costis validis, obliquis, leviter arcuatis, subirregularibus ornati; ultimus altitudinis quintam partem pauluhm superans, inferne filo-carinatus, subtus subplanatus, costulis in strias transformatis. Columella curvata, involuta, callo albo-marginata, oblique truncata, basin attingens. Apertura subrerticalis, subtetragona, intus coerulescente-albirla; peristoma simplex, acutum. marginibus callo tenui, albescente junctis, dextro regulariter arcuato, basali subrecto, fere horizontali.

Long. vix 10 mill.; diam. $2^{2} 3$; apert. lat. $1^{1 / 3}$; alt. 2 mill.

Habitat in Camerunia: Kitta, Ekundu-Etitti (D.). 
Specimina 23 , quorum 10 adulta, omnia viventia collecta, in coll. mea exstant.

Par sa taille et sa grandem cette petite espèce se rapporche beauroup de I'seurlogrlesisula fuscidula (Mor.), mais aprés m examen attentif. elle s'en distingue ausitot par des particularités qui en font une très bonne espèce.

Sa couleux est d'un brun plus foncé, tirant sur le rougeâtre au lieu du verdatre. Son test est plus solide et la sculpture est plus fortement accusée, excepté sur le sommet où elle est pareille chez toutes les denx espèces; les côtes sont plus espacées et moins arquées et obliques. La surface manfue de l'apparence sovense dr l'autre espece et les côtes, étant chez elle de la même couleur que celle du fond, sont chez notre espèce d'une nuance plus foncée que le reste de la surface. La coquille est à peine transparente et l'intérieur de l'ouverture est distinctement teinté de blanc.

La forme entière est plus étroite que celle de la précélente espèce. les touss s'accuissant encore plus lentement et itant moins hants, d'oì il suit que Psendoglessula Dusini fompte un plus grand nombre de tous qu'un expmplaime de Ps. fuscidula de même grandeur.

La base dr dernier tour est moins prolongée, ce qui rend la carène plus saillante et l'ouverture moins haute, presque tétragone. Le profil de la base, convexe chez Ps. tuxcidula, devient cher notre sejere concave par le prolongement de la columelle.

La sculpture de la base est plus affaiblie et la couleur y est de même plus pâle.

Achatina muscorum Moreler, qui probablement appartient au gente P'seubloglessula. est sans nul doute de mène assez voisine de notre espèce, tout en offrant cependant, d'a press la liasmose st les tigures, des partioularités yui l'en distinguent bien.

Nous devons cette élégante espèce à M[. Dusés, auquel nous la dédions. Il l'a trouvée seulement deux fois, l'une sur des plantes, l'autre sur un coquille d'oeuf. Elle paraît être tres rare. 
BIHANG TILL K. ST. VET.-AKAD. HANDL. BAND 22. AFD. IV. N:0 2. 109

\section{Pseudoglessula involuta (Gould).}

1843 Achatina iuvoluta Gould in Proc. Bost. Soc.; Nov., p. 158.

1846

1847

Fraseri Pfenffer Symbolæ III p. 90.

Philippi Abbild. II p. 216, Ach. T. 1, Fig. 6.

Pfajfare Mon. Hel. II p. 263.

1848

1849

Reeve Conch. icon. Vol. Y Pl. 16, N:o 71.

1850 Subulina Fraseri Albers Heliceen p. 195.

1853 Achatina involuta Pfeiffer Mon. Hel. III p. 500.

1855 Subulina Fraseri ADAms H. et A. Gen. of rec. Moll. Vol. II p. 110 .

Achatina involuta Pfeiffer in Chemn. ed. Küster p. 343, T. 37, Fig. 21, 22.

1860 Glandina

(Varicella) Alekrs Heliceen ed. v. Martens 1. 31 .

1868 Achatina

1881 Stenogyra

Morklet Voy. Welwitsch 1\% 80.

1893 Subulina

(Subulina) Pfeiffer Nomenclator p. 325.

v. Martens Mitheil. aus d. d. Sehutzgeb. Band VI, Heft. 3.

Testa turrito-subulata, apice (ylindrico, obtusiusculo, sub)papillari, irregulariter striata, polita, albido-rirescens, sub epidermide corneo-hrumea, hic illic saturatius strigata, in parie aperturali sepius deterenda, sub lente vix perspicur spiraliter densissime striata; spira apicem versus subconcara. ceterum subcylindrica; anfractus 15, primus et secundus suhglobosi, leves, sutura regulariter et distanter denticulatocremulata discreti, sequentes plano-convexi ad suturam irregum lariter plicato-crenulati, ultimns ${ }^{1} 1$ longitudinis non axquans, inferne subangulatus, angulo saturatiore; colmmella arcuata, abrupte truncata, albo-marginata; apertura subtetragonosemiovalis; peristoma tenue, rectum, simplex.

Long. 31-321/2; diam. 6; apert. long. 6, lat. $3^{1 / 2}$ mill.

Ova 3 mill. longa, 2 mill. lata.

Habitat in Camerunia: Bonge (D., S.), Itoki (S.).

(Cap Palmas; in ripis fluvii Nun Afr. Oce. (FRASER);

Sierra Leone: prope Freetown (WeLwitsci);

Togoland: Bismarckburg (BüTTNER).)

Specimina 49 exstant, omnia viventia collecta. 
Tous les savants ont mutierement negligé la rlifference yni "xiste ente la seulpture embryonnaire et relle du reste du test. Il est tres etrmmant que MI. PFfrffer et plusieurs auteurs avec lui aient pu la qualifier de: sutura lavis: MII. Gortu et ReEvesomt les seuls yui parlent dime faible "renelure, mais ils nont pas nom plus remaryue l'apparence differente et singruliere de la sculpture suturale des toms de -pire embryomaires. (1) cest justement cette particularite qui nous a forcé de ranger cette espèce parmi les Psendo-

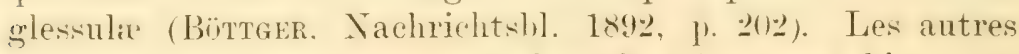
"alartires de lespece correspondent de méme asse\% bien avec ce genre, comme, p. ex., l'angle du dernier tour de spire, la prete particlle de l'épiderme sur la paroi de l'umrerture et la forme oblongue des oents.

Evidemment, les types de M. PFriffer n'me pas atteint leur complet développenuent. Les figures quen ont domnées IIII. Reeve (1. ‘. Pl. 16, N:0 71), Phisppi et Küster, représentent de même de jemes individus. L'exemplaire reprorluit par M. REwry rans la planche 17. X:o 88, de sa Conchologia iconica, ressemble au contraire parfaitement aux nôtres.

Les oeufs que l'on aperçoit à travers le test sont de moitie plus lomgs que larges, et ils ont dans l'oviducte la même prosition oblique que nous arous mentionne chez plusienrs especes précedentes comme mu trait caractérisant lo genre.

Psendoglessula involuta vit, comme ses cong'enèes, sperialement prami les fenilles mortes dans les bois sombres et lnumides, mais on la trouve aussi yuelquefois sur le sol parmi les graminées.

\section{Genus Homorus Albers.}

\section{Homorus pileatus (v. MARTENs).}

1876 Stenoryra pileata v. Martens Monatsber. Berlin p. 261, 'T. 3, Fig. 12, 13.

$1891 \quad>\quad$ Sitzungsber. Gesellsch. naturf. Freunde, Febr. 1. : :il.

Kaméroun: Bonge (S.); Bibundi (J.); (Bonjongo (B.); Buea, Barombi (Pr.)).

7 spécimens. 
BIHANG TILT K. SV. VET.-AKAD. HANDL. BAND 22. AFD. IV. N:0 2, 111

Un seul individu provenant de Bonge, qui compte 11 tours de spire, atteint la longueur extraordinaire de 33 mill. et demi.

Les oeufs sont à peu près sphériques, de 2 mill de diamètre.

Par la sculpture de l'apex, qui diffère de celle du reste de la coquille. rette espère semble voisine du genre Psendoglessula, mais la forme des neufs et la conleur du test ne permettent pas de l'y ranger.

L'espèce a été trouvée avec Subulina angustior DoHrN parmi des feuilles mortes sons un cotonnier (Eriodendron anfractrosum D. C.).

\section{Genus Subulina BEck.}

\section{Subulina angustior DOHRN.}

1847 Achatina striatella Pferffer in Philippi Abbild. II p. 216 T. 1, Fig. 7.

1848

1855

1858

1866

1868

1876

1881
Mon. Hel. 1I, p. 263.

in Chemn, ed. Küster p. 341 Bul.

T. 37, Fig. 17, 18.

var. Moretert Séries conch. I p. 26.

Stenogyra angustior (Suhulina) H. Domns Malak. Blätter XIII 1. 127.

Achatina

Pfeifer Mon. Hel. VI p. 236.

v. Martens Monatsber. Berlin p. 262; T. 3, Fig. 14, 15 .

(Subulina) PFEIFFER Nomenclator p. 327.

Kaméroun: Kimmbe, Lue, Batoki, Ekmmba-N'dene (D.); N'dian (S.); Bonge (D., S.); Bibundi (D., S., J.); (Bonjongo (B.)).

(Ilha do Principe.)

Environ 600 spécimens, recueillis à l'état vivant.

Cette espèce, dont nous avons à notre disposition de grandes masser provenant de plusieurs localités différentes, paraît être assez variable au point de vue de la forme, plus ou moins subulée, avec le dernier tour plus ou moins développé, et tous les tours de spire plus ou moins aplatis. Elle paraît autant variable quant à la couleur, 
grisâtre chez quelques-mns, plus blanchâtre chez d'autres.

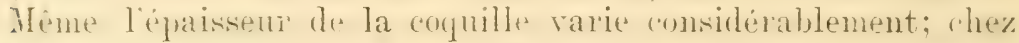
lus exmplaires raiss, yni sont an reste ordinairenent moins étirés en longueur, elle est d'ordinaire très mince.

Il serait peut-être possible de distinguer chez nos exemplaires, sinon denx espexes, dn moins deux varictes, dont l'ume se rapproche de Sulutina striatella (RANi), wt dont l'antre. par sa forme et par sa suture évidemment crénelée, semblerait être assez roisine de S. gracilenta (Iorecer). Nous n'osons tontefois pas nous prononcer, vu le manque de matériaux de comparaison.

Presque tous les exemplaires, qui ont été recueillis en juillet-octobre, même les petits, ne mesurant que 12 mill., sont remplis l'ount's blanes sphrignes, rangis mu denx sérirs. Inisant à traver's le test, et ayant environ 1 mill. de diamètre. Un exemplaire d'environ 28 mill. de longueur, où il ne se trouvait que des débris de l'animal, contenait un oeuf de $1^{1 / 2}$ mill de diamètre et 5 petits embryons avec suture asiez profonde, trés élégamment crónelée sons le microscope. mais à tous autres égards parfaitement lisses et luisants.

Voici ce que M. SJüstedt mentionne quant à son habitat: »A la ferme de Bonge, je trouvai, à une occasion, cette espèce en nombre (60 ex.). Elle se tenait principalement parmi les feuilles tombées sous un cotonnier soyeur (Eriodendron anfractuosum D. C.), ainsi qu'au bas du tronc. Elle

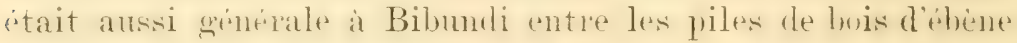
entassées sur la terrasse de la factorerie. Le sol ou elle se tenait, se composait d'un gravier fin de lave noire. On la trouve aussi bien dans les prairies que dans les forêts humides et sombres, sous les feuilles pourries.

\section{Genus Opeas Alibrs.}

\section{Opeas egens n. sp.}

Testa minute perforata, turrita, dense arcuatim plicatostriata, pallide cornea, temuis, diaphana. (ereo-nitens; spira tourita, apice obtusulo; antiautus li; embryonales sub lente minntissims densissimepue spiraliter striati, convexiusculi; senpentes sulpulanati, plus minuses gradati, regulariter ac- 
crescentes, sutura impressa, sub lente subtiliter crenulata et obtuse marginata discreti; ultimus $2 / 5$ longitudinis adiequans, ad suturam angulatus rel gradatus et circa perforationem compressus; apertura oblonga, verticalis; peristoma simplex, marginibus callo tenuissimo junctis, dextro arcuatim producto, recto, columellari longius subfornicatim reflexo; columella subverticalis, plerumque ad sinistram paululum devians.

Long. 5-6 mill.; lat. $1 \frac{1 / 2-2}{2}$ mill.

Habitat in Camerunia: Kitta (S.).

Specimina permulta in alcoh. servata.

Il est probable que cette nouvelle espèce se rapproche très sensiblement d'Opeas pauper DoHrn (MIal. Blätt. XIII, 1866 , p. 126, T. V, Fig. 14-16), mais la forme de la coquille est beancoup plus étirée en longueur, et les tours de spire, plus étagés, sont séparés par une suture profonde, faiblement crénelée et plus ou moins distinctement marginée, caractères dont l'ensemble justifie sans nul doute la création d'une nouvelle espèce.

Nous ne trouvons unlle part mentionnée, comme caractérisant le genre, la sculpture spirale des tours de spire embryonnaires. Elle est d'mne extrême finesse, et ce n'est qu'à une forte loupe et à une lumière favorable qu'on l'aperçoit distinctement. Nous n'avons pas hésité, néanmoins, à ranger notre espèce dans ce genre, car nous sommes convaincu qu'on retrouvera la même sculpture chez tous les Opeas.

Presque tous nos exemplaires sont munis d'oeufs parfaitement sphériques et rangés en une série simple dans l'oviducte, ou ils sont visibles par transparence à travers la coquille.

Opeas egens a été recueilli parmi des feuilles pourries. 


\section{Fam. SUCCINEID王.}

\section{Genus Succinea Draparnaud.}

\section{Succinea concisa MorELET.}

\begin{tabular}{|c|c|c|c|}
\hline 1848 & Succinea & concisa & Morelet in Revue zool. p. 351. \\
\hline 1850 & $\gg$ & spurca & Gould in Proc. Bost. Soc. III p. 193. \\
\hline 853 & $\gg$ & $\gg$ & Pfeiffer Mon. Hel. III p. 12. \\
\hline$\gg$ & $\Rightarrow$ & concisa & $\gg \quad \gg \quad \gg \quad$ p. 11 \\
\hline 1854 & $\gg$ & 》 & $\begin{array}{l}\text { in Chemn. ed. KÜSTER p. } 46, \text { T. } 4 \text {, } \\
\text { Fig. } 44-46 \text {. }\end{array}$ \\
\hline 855 & 》 & $\gg$ & ADaMs H. et. A. Gen. of rec. Moll. II p. 128. \\
\hline & 》 & spurca & $\gg \gg \gg \gg \gg \quad \gg \quad \gg .129$. \\
\hline 58 & $\gg$ & coucisa & Morelet Séries conch. I, p. 11, Pl. 3, Fig. 7. \\
\hline 6 & $》$ & spurca & Albers Heliceen ed. v. Martens p. 310. \\
\hline 366 & $\gg$ & eoncisa & H. Dourn Malak. Blätt. XIII p. 133. \\
\hline & $\gg$ & $\gg$ & Morelet Voyage Welwitsch p. 44. \\
\hline & y & $»$ & Pfeiffer Nomenclator p. 413. \\
\hline & $»$ & spurca & $»$ \\
\hline
\end{tabular}

Kamérom: Bibundi(D.,S.,J.); Batoki, Ekumba-N'dene(D.). (Liberia, Ilha do Principe, San Thomé, Gabon.)

Plus de 200 spécimens, conservés dans l'alcool.

Les spécimens que nous avons sous le yeux ne correspondent pas exactement aux diagnoses qui ont été données de cette espèce, mais les différences qu'ils offrent ne sont pas assez importantes pour les en séparer.

Parmi le grand nombre qui s'en trouvent à notre disposition, aucun ne compte plus de $2^{1}$ ' tours de spire; pas même les exemplaires de la grandeur indiquée par M. Monelet n'atteignent les 3 à 4 tours qu'il mentionne pour son espèce. Les stries d'accroissement, que MI. Monelet trouve a peine perceptibles à l'oeil nu, sont chez les nôtres aisément risibles sans l'aide de la lonpe; un trait, au contraire, dont ne parle pas ce savant, et que nos exemplaires partagent avee $\mathbf{S}$. modesta Goud, sont des sillons spiraux interrompus, plus ou moins distinctement marqués.

Selon M. Pfeiffer, la suture de s'. concisa Morelet est ssubmarginata et la columelle superne subcallosas, mais un 
examen attentif ne nons l'a pas fait constater (hez nos spécimens; la columelle différe aussi de celle de S. spurca, en ce qu'elle n'est pas fortement arquée.

Nous ne trouvons nulle part mentionné que S. concisa se couvre de terre comme le fait S. spurca Gould; mais, à en juger par la diagnose: sstriis laxis scabris lutum cumulantibus, l'enduit, chez celle-ci, ne peut pas être disposé de la manière singulière que présentent nos sujets, ou il forme trois hautes carènes spirales parallèles à la suture; on rencontre cette singularité chez tous nos exemplaires, grands to petit., quoiqu'ils proviennent de localités assez distantes l'me de l'autre, Bibundi et Batoki étant situés sur la côte. EkumbaN'dene à environ 35 kilomètres dans l'intérieur du pays.

Comme M. MLorelet, en la citant dans la synonymie (Séries 1. c.). approuve la diagnose ru'a dommée M. Pferffrer de S. concisa MoReLet, malgré les différences qu'elle présente, et (qu'en outre il identifie S. spurea (Gould avec son espece (Vor. Welw. 1. c.), opinion que M. Pfemfer semble partager, nons avons hésité à créer une nourelle espèce de nos exemplaires, quoique les particularités dont nous avons parlé ci-clessus nous y autorisassent peut-être.

L'espèce a été recueillie pendant les mois do juillet-septembre; la plupart vivaient sur des trones pourris de palmiers. MI. Dusén a même trouvé plus de 100 individus sur un seul tronc.

\section{Fam. VERONICELLIDÆ.}

\section{Genus Veronicella Blatnville (emend.).}

Veronicella pleuroprocta V. MARTENS.

1876 Veronicella pleuroprocta v. MarTens Monatsber., Berlin, p. 268. T. 5, Fig. $2-5$.

Kaméromn: N'dian, Kitta, Itoki (S.), Bonge, Bibundi (S., D). (Barombi $(\mathrm{Pr})$.)

(Côte d'Or: Aburi (B.); Liberia (Benson); Togoland: Bismarckburg (BüTTNER).)

Spécimens en abondance. 
Le grand nombre d'individus provenant de plusieurs lowalitis différentes que nons avoms devant nous, renferme sans nul donte plusieurs especes, mais n'ayant ni litterature, ni matérianx de comparaison. nous n'osons pas les séparer.

MI. SJöstedr nous écrit sur l'occurrence de cette espèce: :Te la rencontrai surtout en abondance en août pendant la saison des pluies a la ferme de Bibundi, converte de l'herhe à éphant (Pennisetum polystachyon Schult.) et d'une zingihéracée (Amomum granum paradisi L.). On l'y aurait pu recueillir alors par milliers sur les fenilles. Et ML. Dust. declare quelle est excessivement commune sur l'Amomum et sur les graminées etc., mais non sur le sol.

\section{Fam. AURICULID坐.}

\section{Genus Melampus Montrort.}

\section{Melampus flavus (GMELIN).}

1791 Toluta flava Guelin Syst. p. 3436 N:0 5.

1792 Bulimus monile Bruguière in Encycl. méth. I p. 338 N:o 70.

1839 Conovulus flavus AntoN Verzeichn. p. 48 N:o 1776.

1844 Auricula monile KüsTer in Chemn. ed. Küster Auric. p. 30, T. 4, Fig. $7-9$.

1856 Melampus flavus Pfeiffer Monographia Auriculaceorum p. 21.

$1866 \gg$ \#. DoHRN in Malak. Blätter XIII. p. 133

Kaméroun: Jonje (D.).

(Illha do Principe (DoHrs); Antilles.)

10 spécimens conservés dans l'alcool.

Tous nos exemplaires se distingnent par len couleur chatain singulièrement foncée. Les trois handes claires, de la largeur d'un fil, sont faiblement prononcées. A l'exception de la région très luisante à la paroi de l'ourerture. toute la coguille est recourerte d'un mince épiderme coulenr olive, qui ne laise que tris faiblement entreroir les bandes claires.

Chez aucun de nos exemplaires la suture ne présente de hord clair. La seulpture spirale, qui est le plus fortement accusée vers la base et à la partie supérieure de la 
BIHANG TILL K. SV. VET.-AKAD. HANDL. BAND 22. AFD. IV. N:0 2. 117

spire, s'apercoit néanmoins aussi, sous un fort grossissement et à une lumière favorable, sur les autres parties de la coquille.

Cette espèce habite les cavités de tuf basaltique atteintes parfois par les brisants.

Melampus Liberianus $H$. et A. ADAMs.

1854 Melampus Liberianus Adars H. et A. in Proc. zool. Soc. p. 12. obovatus

》 ibidem.

Pfeiffer Novitates Conch. I p. 21. T. 6, Fig. $10-12$.

$1856 》 \gg$ Monogr. Auricul. p. 23.

》 $\gg$ obovatus 》 》 $\gg 46$.

1878 \iberianus H. DOHRN in Jahrbücher Malak. Gesellsch. p. 151 .

Kaméroun: prés du fleuve Oonge (J.).

(Liberia; Côte d’Or.)

20 spécimens conservés dans l'alcool.

Nos exemplaires correspondant en tout avec la description qu'a donnée Preiffer de Melampus obovatus $\mathrm{H}$. et $\mathrm{A}$. Adams, lequel, suivant M. Dourn est la forme non adulte de II. Liberianus, nous n'avons, pas hésité à donner cette espèce sous le nom mentionné en tête, quoique manquant de matériaux de comparaison.

Le bord extérieur de l'ouverture a chez tous une bordure claire, et des exemplaires de la même grandeur, 8 mill., sont munis à l'intérieur, à une certaine distance du bord, de 1 , 2. 3, jusqu'à 12 lamelles, ou aussi en manquent totalement, circonstance qui milite ultérieurement en faveur de l'opinion de M. Dohrn.

Outre la lamelle saillante dans la partie inférieure de la paroi de l'ouverture, s'étendant en spirale dans l'intérieur de la coquille, les parties moyennes montrent quelquefois une callosité tuberculoïde, qui chez quelques exemplaires est nettement divisée en 2 on 3 dents. Le nombre des bandes pâles varie de 1 à 4 . Un exemplaire n'en porte aucune.

Cette espèce a été recueillie sur des points inondés par des eaux saumâtres près du fleuve Oonge. 


\section{Gemus Tralia Gray.}

Tralia pusilla (GMELIN).

1791 Voluta pusilla Guelin Syst. p. 3436. N:o 7.

1792 Bulimus ovulus Bruguière in Encycl. méth. I. p. 339. N:o 71.

1844 Auricula nitens KUster in Chemn. ed. KÜs. p. 18. T. 2. Fig. $11-13$.

1853 Conovulus pusillus Forbes et Hanley Brit. Moll. IV. p. 197.

1855 Tralia pusilla Adans H. et A. Genera of rec. Moll. II. p. 244.

T. 82, Fig. 8 .

1856 Melampus pusillus Pfemfer Monogr. Auricul. p. 46.

1866 》 $\gg$ H. DoHre in Malak. Blätt. XIII. p. 133.

Kamérom: Jonje (D.).

(Ilha do Principe (Domrx));

(Antilles; Sandwich, Oahu.)

17 spécimens conservés dans l'alcool.

Les exemplaires que nous avons sous les yeux paraissent se séparer légèrement de ceux que nous possédons de diverses régions des Indes occidentales. La spire est plus courte, d'ou il suit que le dernier tom est plus fortement anguleux vers le haut, ot la couleur est d'un chocolat foncé, non châtaiu. La coquille est recouverte d'un épiderme vert jaunâtre se ditachant aisément ef prenant alor's me couleur tirant sur l'or. Cet épiderme s'étend juspu'au callus nettement limite et très brillant qui réunit les hords de l'ouverture. La spire présente chez tous les exemplaires des érosions plus on moins semsihles, et le dernier tom ottre des lignes de croissance grossières avec de fortes érosions.

L'esperce a été tronvée arec Melampus flarus daus les mêmes cavités de tuf basaltique.

\section{Genus Pedipes Adanson.}

Pedipes Dohrni n. sp.

Testa imperforata, globoso-conica, solida, liris decurrentibus, validis, confertis, prope suturam latioribus ibique angustioribus hic illic interjectis, seulpta, rufo-fusea; spira sub- 
gradato-conica, apice acutiusculo, levigato; anfractus 4, superi superne distincte angulati, supra angulum leviter excavati, ultimus $2 / 3$ longitudinis subrequans, superne basique obtuse rotundato-angulatus, angulo basali paululum distinctiore; apertura diagonalis, semicircularis, lamella parietali, alba, obliqua, compressa, subtus excavata, valida, intrante et dentibus columellaribus 2 albis, quorum superior lamelliformis, spiraliter intus continuatus, inferior minor, acutus, munita; peristoma acutum, marginibus callo tenui junctis; dextro medio intus tuberculo, deorsum in callositatem longitudinalem continuato, instructo; columellari calloso, nitido, leviter excavato.

Long. 5, lat. 4 mill.; apert. $3^{1 / 2}$ mill. longa, 2 mill. lata. Habitat in Camerunia: Jonje (D.).

Specimina 12, viventia collecta.

Comme on le voit par la diagnose qui précède, cette espèce se rapproche non-seulement de Pedipes mirabilis (MÜHLFELD), mais aussi de P. globulus Petit et de P. angulatus C. B. AdAus. Malheurensement nous n'avons à notre disposition aucune de ces trois espèces, mais les particularités les différenciant de la nôtre qu'offrent les descriptions, ne nous permettent pas d'identifier celle-ci avec aucune d'entre elles.

Elle a été recueillie par MI. Dusén, en 1891, avec Melampus flavus (Guesis) et Tralia pusilla (GMeLis) à Jonje dans des cavités de tuf basaltique, parfois atteintes par les brisants.

M. H. Dohrn mentionne (Malak. Blätter XIII, 1866, p. 134) une espèce du genre Pedipes, qu'il a trouvée à l'île du Prince en compagnie des mêmes espèces avec lesquelles la nôtre a été recueillie, et qu'il déclare être probablement nouvelle, mais qui a été perdue avec d'autres parties de ses collections. Comme il est possible que c'était l'espèce décrite par nous que l'illustre savant a eue entre les mains, nous la lui dédions. 


\section{Fam. CYCLOPHORID $¥$.}

Genus Cyclophorus Montfort.

\section{Cyclophorus Preussi V. Martens.}

1892 Cyclophorus preussi v. MarTexs Sitzungsber. Gesellsch. naturf. Freunde, Berlin, Nor., p. 183.

Kaméroun: Buea (J.; Pr.), Mokundange (D.).

Deux spécimens, dont l'un conservé dans-l'alcool.

La sculpture des premiers tour's de la spire, qui en compte un de plus que ne lui en reconnait M. V. Martexs, rrésente quelques particularités dont ce sarant ne parle pas, et qui ne sont visibles qu'à la loupe. L'un de nos deux exemplaires étant eu très bon état, cela nous permet de faire les observations que voici.

L'apex étant presque lisse, les denx premiers tours de spire sont ornés de côtes pliciformes, minces et serrées, plus fortement accusées au-dessous de la suture, qui, après un affaiblissement success if et presque entier sur le troisième tour, se transforment peu à pen en côtes fortes et régulières couvrant le reste de la coquille. En outre, les tours embryonuaires sont ornés de stries filiformes décurrentes très nettement accusées et assez espacées entre elles, s'évanonissant aussi sur le troisième tour. On aperçoit de même ces stries au côté opposé des tours au fond de l'ombilic. Le dernier tour s'abaisse quelque peu rers l'onverture exactement circulaire.

L'm des exemplaires que nous arons derant nous et qui est de la même grandeur que celui décrit par MI. v. Mantexs, est anssi de la même origine: il a été recueilli à Buea, en juin 1R91. sous des feuilles mortes par II. le Dr. Juxgaen, 'qui l'a ainsi probablement déconvert le premier; l'autre, qui a senlement \& mill. de diamètre, provient de Mokundange (ou Makondanye) situé sur la côte de la baie d'Ambas, a l'onest de Victoria. 


\section{Fam. CERITHIID王.}

\section{Genus Potamides Brongniart.}

\section{Potamides (Tympanotonos) fuscatus (LINNÉ).}

1767 Murex fuscatus Linné Syst. nat. ed. XII p. 1225.

1791 》 cingulatus GMeLin Syst. nat. ed. XIII p. 3561.

1792 Cerithium muricatum Bruguire Encycl. méthod. I, p. 490, N:0 27.

1887 Potamides (Tympanotonos) fuscatus Tryon Man, of Conch. P. 35 , p. 159 , T. 31 , Fig. 34 .

Kaméroun: Ekundu-N'dene (D.).

(Gambie, Cap Palmas.)

11 spécimens, conservés dans l'alcool.

Liespèce était très commune dans les eaux sammâtres.

\section{Potamides (Tympanotonos) radula (LiNNÉ).}

1767 Murex radula LInné Syst. nat. ed. XII p. 1226.

1780 Turbines circulis etc. (Cerithium Owenii Férussac) Martini Conch. p. 304, T. 155. Fig. 1459 .

1887 Potamides (Tympanotonos) radula Tryon Man. of Conch. P. 35. p. 159 Pl. 31 , Fig. 35, 36 .

Kaméroun: la localité non précisée.

Un seul exemplaire avec qui concorde le mienx la figure donnée pour Cerithium Owenii FÉR. (1. c.).

\section{Fam. MELANIID王. \\ Genus Melania Lamírck. \\ Melania Nigritina MoreleT.}

1848 Melania nigritina Morelete in Revue zool. p. 355.

1851 》nigrita 》 \Journ. Conch. II. p. 191 Pl.5, Fig. 2.

1858 » $\gg$ igritina $》$ Séries conch. I. p. 31. Pl. 3, Fig. 8.

1860 foenaria Reeve Conch. icon. Vol. XII, N:o 134. 


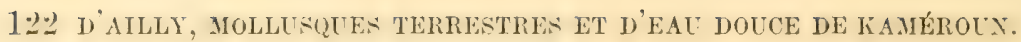

1874 Melania nigritina BroT in Chemn. ed. KÜster p. 67 'T. 7 , Fig. 8 .

var. $\beta$ Вrот 1. c. p. 68 , T. 7 Fig. 8 a, 8 b.

1876 \igritina v. Martens Monatsber., Berlin p. 270.

Kaméroun: Bibundi: fleuve Bekongele; Basse, Etome: fleuve N'dive; Boangolo: Aleuve Jongalove; Loe: dans le erique (D.).

(Calabar; Gabon.)

(Bonjongo (B.), Barombi (Pr.))

Spécimens en abondance, conservés dans l'alcool.

Parmi les riches matériaux que nous avons devant nous de cette espèce, on peut distinguer la varièté $\beta$ Brot; la variété $\gamma$, au contraire, n'y est pas représentée.

Une grande partie de nos exemplaires présentent sur le milieu des tours une zone foncée. Le dernier tour en porte une seconde an-dessous de l'augle de la périphérie; elles correspondent aux fascies de l'intérieur de l'ouverture. Ces exemplaires ont ordinairement la suture sous-bordée d'une nuance pâle, circonstance qui les rapproche de Melania foenaria Reeve (l. c.). Sous l'épiderme, le test est d'un blanc lilas clair qui devient pur au-dessons de la suture, et qui produit, par transparence, la pîle bordure suturale dont nous venons de parler; les bandes y sont d'un lilas foncé.

Melania Nigritina est trés commune dans les torrents et les courants rapides du voisinage de la mer, mais ne se rencontre jamais dans les eaux calmes.

\section{Genus Claviger Haldemann.}

\section{Claviger Matoni (GRAY).}

1791 Murex fuscus GMelin Syst. nat. ed. XIII. p. 3561, N:0 139.

1831 Melania Matoni Gray Zool. Misc. p. 10.

$1843 \gg$ fusca Philippi Abbild. I. p. 59. T. 2, Fig. 1.

$1860 \gg$ 》 $\gg$ REvE Conch, icon. Vol. XII. N:o 200.

1874 Claviger Matoni Brót in Chemn. ed. Kúster p. 366, T. 37 Fig. 3, 3 a, $3 \mathrm{f}$.

1876 Melania (Vibex) fusca v. Martens Monatsber., Berlin, p. 270. 
BIHANG TILL K, SV. VET.AKAD, HANDL. BAND 22. AFD. IV. N:0 2. 123

Claviger Matoni var. $\beta$ Brot.

1831 Melania quadriseriata Gray Zool. Mise. p. 10,

1843 》 mutans Gould Proc. Bost. Soc. p. 159.

1850 》esselata LEA Proc. zool. Soc. London p. 192.

$1860 \gg$ mutans ReEve l. c. N:o 215.

1874 Claviger Matoni var. $\beta$ BRot 1. c. p. 367 . T. 37 , Fig. $3 \mathrm{~b}-\mathrm{e}$, 4,4 a, b.

Kaméroun: Bibundi: fleuve Bekongele (D., S.), EkunduN'dene: crique près du fleuve Meme (D.). (Victoria (B.).)

(Senegal; Calabar.)

Nous avons de chacune des différentes formes de cette espèce protéeune un très grand nombre d'exemplaires, qui prouvent indubitablement l'identité des formes, que l'on prendrait an premier aspect pour des espèces très distinctes. Ainsi toutes les rariations de formes sont représentées parmi les exemplaires provenant du fleuve Bekongele à Bibundi.

\section{Fam. AMPULLARIID王.}

\section{Genus Lanistes MontFort.}

Lanistes Libycus (Morelet) et var. Bernardianus (Morelet).

1848 Ampullaria Libyca Morelet in Revue zool. p. 354.

1851 》 Philipp in Chemn. ed. Küster p. 25 T. 6. Fig. 8.

$1858 》$ Morelet Séries conch. I p. 28, T. 3, Fig. 9. 1876 Lanistes Libycus v. Martens Monatsber. Berlin p. 270.

var. Bernardianus (Morelet).

1860 Ampullaria Bernardiana Monelet in Journ. de Conch. VIII p. 190. 1866 Lanistes Bernardianus var. v. Martens in Novitates Conch. II p. 286 T. 70 , Fig. $1-4$.

Kaméroun: Boangolo: fleuve Jongalove (D.); Bonge; Bibundi: fleuve Bekongele (S.); (Victoria (B.), Barombi (PR.)).

(Gabon; Afrique centrale (Schweinfurth).) 
124 D'MLLY, MOLLUSOLES TERREATRES ET D'FAU DOUCE DE KAMÉROTN.

la variété: Kaméroun: Kumbe, Etome: fleuve N'dive (D.); Bonge (S.). (Lagos.)

Spécimens en grande quantité des deux formes.

On considère actuellement comme synonymes Lanistes Libyrus (Moriset) et Lanistes Bernardianus (Monelet). L'étude rle la grande quantité d'exemplaires qui se trourent a notre disposition wous a fait toutefois arriver à une opinion différente. Parmi ces exemplaires, il s'en trouve qui correspondent en tout aux descriptions domées des deux espèces, et qui nous paraissent devoir être considérées an moins comme variétés. Un fait certain, c'est que quiconque comparerait des exemplaires appartenant le plus typiquement aux deux formes, les attriburait sans hésitation à des espèces parfaitement distinctes. Les denx types sont néanmoins reliés entre eux par des formes intermédiaires qui, selon nous, peuvent difficilement justifier me distinction pareille.

Le caractere le plus distinctif de L. Bernardianus, est sa soulpture spirale, it stries filiformes de force et a intervalles variables. L'me de ces stries occupe l'angle supérieur même des tours de spire, et lui donne l'apparence d'une carène en forme de cordon. ('es stries peuvent rependant se rencontrer sur les parties plus récentes de la coquille, tandis quelles s'aff'aiblissent on disparaissent totalement à une période postérieure re croissance. Elles manquent au contraire completement chez la forme principale, on elles n'y sont que légèrement accusées sous l'aspect de faibles séries spirales a apparence granulée. Dans la forme principale, la carène n'est pas munie d'm cordon pareil, mais elle off're la forme d'um angle aigu s'arrondissant plus on moins vers l'ouverture chez les grands exemplaires.

La sculpture verticale de ceux de nos exemplaires que nous estimons appartenir à la variété, est anssi en général plus serrée et plus régulière, avec stries de croissance ça et lia plus saillantes. Ils sont aussi revetus le plus souvent d'un épiderme foncé, couleur olive, qui ne laisse que très indistinctement percer les bandes colorées sousjacentes. Aucum d'entre eux n'a me conleur anssi claire que les exemplaires reproduits dans les Noritates (1. c.), couleur qu'ont par contre 
tous les exemplaires que nous possédons de la forme principale, mais se rapprochent davantage, quant à la conleur, des exemplaires domnés au même endroit sons le nom de Lanistes Libyens Mor.?.

Un caractère appartenant tant à la forme principale qu'à la variété, mais qui ne se trouve mentionné nulle part, est l'enveloppe extérieure tonte particulière de l'épiderme, ressemblant à du feutre on à du velours. Elle se compose de lamelles extrêmenent minces, membraneuses, correspondant aux fines stries rerticales sonsjacentes. Partout où elles coupent les stries spirales filiformes, ces lamelles sont étirées en petites pointes on en petites frisures dont l'apparence ne permet pas la dénomination de poils ou de cils, et dont le développement correspond à celui des stries sousjacentes. Il en résulte une quantité de bords frangés spiranx à intervalles variés et de force différente. Or, quelles peuvent être les relations de ces types avec Lanistes ciliatus v. Martexs (Monatsber. Berlin, 1878, p. 296, T. 2, Fig. 8-10)? Des exemplaires de la variété de la même grandeur que le seul exemplaire connu de L. ciliatus, et par conséquent nou adultes, correspondent en tout par leur forme et leur couverture extérieure avec les figures que M. v. Martens a données de cette espèce. Le nombre des bords frangés, qui est de 15 à 16 chez les exemplaires non adultes, est beancoup plus considérable chez les plus grands individus, par la raison que la sculpture spirale qui se voit sous la forme de stries fines entre celles d'apparence filiforme dans les phases plus jeunes de la coquille, continue, pendant la croissance de celle-ci, à se développer partiellement en stries filiformes avec la converture qui les revêt. La circonstance seule que l'auteur ne mentionne pas de bandes colorées, et qui fait supposer que son espèce est unicolore, aimsi que le lieu de la découverte, Finboni, sur la côte de Zanzibar, nous empêchent de la citer dans la synonymie. Chez la torme principale, cette enveloppe extérieure n'est que très rarement conservée, mais elle existe par contre dans la règle chez la variété et principalement chez les exemplaires non adultes. Cette circonstance, jointe i la diversité de la conleur chez les deux formes, dépend sans mul doute de la nature des cours d'ean où on les rencontre. La forme principale semble préférer les eaux claires avec fond de gravier ou de sable. La variété 
se rencontre de son côté dans les eaux à fond de vase ou d'argile. Cette dernière est par conséquent exposée à me nsure moins considérable, ce qui lui permet de conserver son enveloppe extérieure. La rase noire domne aussi me couleur sombre a la rariété. Les exemplaires manquant de l'enreloppe extérieure feutrée, en général adultes, sont enduits en compensation d'une couverte de rase noirâtre très fine et très tenace, cachant les stries filiformes sousjacentes, et prenant un aspect de suie à l'état sec.

Sous l'épiderme, qui chez quelques exemplaires est fortement attaché au test, tandis que chez d'autres il s'y détache si facilement. que quand la coquille s'est séchée après sa sortie de l'alcool, il se brise immédiatement, le test est d'un blanc laiteux chez les deux formes, avec de nombreuses bandes violettes d'intensité différente, qui principalement dans la partie supérieure des tours de spire et surtout chez la variété, confluent plus ou moins complètement, et sont tout particulièrement visibles dans l'intérieur de la coquille.

Ces mollusques déposent leurs agglomérations d'oeufs sur les plantes aquatiques. Une agglomération pareille que nous avons dans de l'alcool, se compose de 15 oeufs, de forme sphérique, à diamètre de $3^{1}, 2$ à 4 mill., et consistant en une membrane assez forte, de la transparence du verre, a travers laquelle on voit l'embryon. Ce dernier, qui compte un tour de spire, est finement strié en spirale sur toute sa surface jusqu'au nucléus, et tant l'angle supérieur que la carène ombilicaire s'accusent avec une pleine évidence.

\section{Fam. NERITID丑.}

\section{Genus Neritina Lamarck.}

\section{Neritina Oweniana GRAY.}

\section{Neritina Oweniana Sowerby Conch. illustr. Fig. 15.}

1855

1856
Thesaurus conch. II p. 519, Pl. 114, Fig. 168.

Reeve Conch. icon. Vol. IX., Pl. 13 N:0 59. GraY in Wood Index Testaceologicus, Suppl. p. 232 Fig. 8. 
BIHANG TILL K. SV, VET.-AKAD. IIANDL. BAND, 22. AFD. IV. N:0 2. 127

1879 Neritina Oweniana v. Martens in Chemn. ed. Kobelt p. 75 T. 9, Fig. $14-17$.

1887

Tryon Man. of Conch. P. 38. p. 76, Pl. 22.

Fig. 90 .

Kaméroun: fleuve N'dian, Itoki: fleuve Masakke (S.).

(Fernando Po (Fraser); Cap Palmas.)

60 spécimens.

II. v. Martens déclare, dans sa monographie, que l'apophyse apicale (der Zapfen) du seul opercule de cette espèce qu'il a en l'occasion d'examiner, manquait totalement, mais la plupart des exemplaires que nous avons examinés la possèdent sous la forme d'un tubercule plus ou moins développé, qui s'étend en arrière dans une courbe parallèle à l'apophyse claviforme, et diminue successivement de hauteur.

M. Sjőstedt nous a communiqué ce qui suit sur la trouvaille de cette charmante Néritine: Elle fut trouvée parmi des galets et du gravier au-dessous de la chute du Heuve N'dian à environ 10 kilomètres de la côte, et au point où la marée cesse de se faire sentir. La trouvaille en est due à nne heureuse circonstance. Quelques indigènes qui, lors d'une course en canot à proximité de la chute, avaient laissé tomber un objet dans l'eau, s'efforcèrent de le repêcher par des plongeons réitérés. Cette Néritine se trouva parmi les galets et les graviers rapportés par eux à la surface. Grâce aux plongeons ultérieurs anxquels on réussit à les engager, les indigènes en question réussirent à ramener un nombre assez grand d'exemplaires pour montrer que l'espèce était commune sur ce point-là.»

\section{Neritina Afra SowerbY.}

1843 Neritina Afra Sowerby Conch. illustr. Fig. 13.

$\gg$ Africana RÉcluz in Proc. zool. Soc. London p. 198.

1848 æquinoxialis Morelet in Revue zool. p. 355.

1850 》 $\gg$ RÉClUz in Jouru. conch. p. 146.

$1855 \gg \quad 》$ Thesaurus conch. II. p. 520, P1. 115,

Fig. 193.

》 Listeri Reeve Conch. icon. Vol. IX. Pl. 15, N:o 71.

1858 æquinoxialis Monet.et Séries conch. I p. 29, Pl. 3, Fig. li. 
12S D'AILLY, MOLLUSQUES TLRRESTRES ET D'EAU DOUCE DE KAMÉROUN.

1866 Neritina xquinoxialis DoHnN in Malak. Blätter XIII p. 134.

1868 \ MORELET Voyage Welwitsch p. 96.

1879 Afra v. Martens in Chemn. ed. Kobelt p. 78, T. 5, Fig. $1-4$.

1887 » T'rYoN Man. of Conch. P. 37 p. 61 (ex parte), Pl. 20, Fig. 42 (nec Fig. 43, 44).

Kaméroun: Bibundi: fleuve Bekongele (I., S., J.), N'dian (D.).

(Fernando Po; Ilha do Principe; Angola.)

\section{5 spécimens.}

La description que donne M. Dours des dents du bord columellaire est parfaitement conforme aux exemplaires que nous possédous. Sauf cela, ils ressemblent en tout aux figures données par MoreLET.

\section{Neritina rubricata Morelet.}

1858 Neritina rubricata Monelet Séries conch. I. p. 30, Pl. 3, Fig. 2. Calabarica Mousson collect. (fide v. Martens).

1876 rubricata $ミ$ Martens Monatsber. Berlin p. 271.

1879 》 $》$ in Chemn. ed. Konelt p. 81, T'. 13, Fig. $9-11$.

1887 》 Afra Tryon Man. of Conch. P. 37 p. 61 (ex parte), Pl. 20, Fig. 43, 44.

Kaméroun: Loe (I).); Bibundi (D., S.): (Victoria (B.)).

(Sénégambie; Old Calabar; Gabon (?).)

Spécimens en abondance.

Les deux variétés de grandeur mentionnées par M. $\mathrm{r}$. MIArtexs, sont representies par de nombrenx exemplaires. Ceux yui ont été recueillis sur les racines des Rhizophorées (Mangroves) ont de plus petites dimensions, et offrent de mrandes varictés de conlen et de dessin. Quelques exemplaires sont d'un rouge brun micolore, d'antres sont ornés de bandes pales on fonceses et de taches a disposition très variable. [ue système de coloration des grands exemplaires, provenant du beach de Loe, est parfaitement semblable a chlui de Neritina Oweniana Grar. Les figures données che\% ('numsitz (l. c.) ne sont pas homes: les taches nont pas leur's 
pointes dirigées vers le haut (voir la fig. 11 l. c.), mais sont d'une forme ovale plus on moins triangulaire aree la pointe toujours dirigée en avant.

11. Tryon a placé cette espèce comme symonyme de Neritina Afra Sowerbr, mais quiconque a en, comme nous, l'occasion de comparer un grand nombre d'exemplaires des deux espèces dans tous les âges et provenant de diverses localités, n'adoptera jamais cette manière de voir. Les particularités qui caractérisent Neritina rubricata Morelet sont très bien décrites par M. v. Martens dans son excellente monographie du genre Neritina. N. Tryon nous semble d'ailleurs trop disposé à réunir comme synonymes des espèces parfaitement distinctes.

\section{(?) Neritina Adansoniana RÉcLUZ.}

1841 Nerita Adansoniana RÉcLUz in Revue zool. p. 313.

1848 Neritina Sangara Morelex ibidem p. 355.

$1855 \gg$ Adansoniana Sowerby Thesaurus II p. 535, Pl. 116, Fig. $254,255$.

1856

ReEve Conch. icon. Vol. IX Pl. 32, N:0 145.

1858

1879

Morelet Séries conch. I p. 30 .

1887

v. Martens in Chemn. ed. Kobelt p. 129 , T. 14, Fig. 22, 23.

Tryon Man. of Conch. P. 37, p. 40, Pl. 13, Fig. 50, 51 .

Kaméroun: Ekundu-N'dene (D.).

(Sénégal; Cap Palmas.)

\section{5 spécimens.}

Nous ne sommes pas sûr que les exemplaires que nous avons entre les mains représentent en effet Neritina Adansoniana Récuuz, que nous ne commaissons que par des descriptions et des figures, mais ils s'en rapprochent du moins de très près.

Ils offrent des dimensions très inférieures à cette espèce, le plus grand ne mesurant que 8 mill. de hanteur sur $8^{1 / 4}$ de diametre max., et leur forme est plus globuleuse. Le systeme de coloration se compose, ontre de fines stries ondulées qui caractérisent $\mathrm{N}$. Adansoniana, de taches triangulaires ou arrondies ou de bandes obliques, d'où résulte une ressemblance évidente arec celui de $\mathrm{N}$. virginea $\mathrm{L}$. La figure 4 


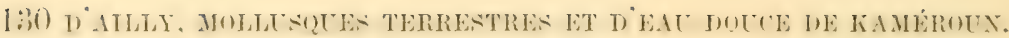

(Chemn. Pl. 14) que Mr. r. MakTtas a domée de cette esperenai resimble parfaitement a quelpues-mu de nos exemplaires. D'antres sont entourés de deux zones foncres yui renferment alors les taches. La rounille est d'm blane de vire avec les stries dun gris plus on moins moiratre. et sil surface offre im éclat mat. Le bord interne de l'area columellaire est muni de 5 a 7 dents fines. dont la plus haute est fortement développée et se prolonge en forme de lamelle dans l'intirieur de l'onverture. On retrouve aussi ce caractère chez $\mathrm{N}$. virginea.

Elle a été recneillie par M. Dusḱn dans de l'eau sanmâtre au fond d'une petite crique mise à sec à la marée descendante, près de la factorerie d'Ekundu-N'dene.

\section{Fam. MYTILID框。}

\section{Subfam. Dreissensiina.}

\section{Genus Dreissensia VAN Benedex.}

\section{Dreissensia Holmi 11. sp.}

(PI. V, Fig. 17-23.)

Comeha triquetra. mytiliformis, antice rotmdato-earinata. margine inferiore umbones rersus angustissime hiante. fusca, epidermide facile deterenda, oncentrice striata. postice squamosa induta: umbones erosi; septum tergum rersus lamina interna, postice excavata instructum.

Long. 16-20; alt. 9-10; lat. $6-7$ mill.

Habitat in Camerunia: Ekumbi (S.).

Cette espèce est sans nul doute assez voisine de Dreissensia Africana Tax Baxbex. provenant du sénégal, mais relativement is la longuenr elle est d'une hautenr beaneoup plus considérable, d'ou il résulte anssi une forme plus triangulaire. La forme de la lamelle dont est muni le septum, diftere ansi chez les deux esperess. ('ette lamelle nous semble assigner id ces deux especes une place prés du sous-genre Mrtilopsis (oxman). qui habite tontefois les tleuves d'Amérique. 
BTHANG TILI K. SV. VET-AKAD. HANDT. BAND 22. AFD. IV. x:0 2. 131

Dreissensia Holmi, que nous dédions à MI. le Dr. Germalid HoLm, paléontologiste suédois, a été recueillie par MI. S.̈̈̈теIт dans des eaux saumâtres.

\section{Fam. CYRENELLID王.}

\section{Genus Cyrenella Deshayes.}

Cyrenella rosea 1 . sp.

(PI. V, Fig. 24-30.)

Concha inflata, subcordata, concentrice leviter striata, umbonibus levibus, plerumque roseis exceptis, sub epidermide squamosa, fuscescente, pallide "ormea vel cerea, intus viola('eo-albida, sursum et antice rosacea; umbones prominuli, antrorsum rersi; dens primus valvie dextre maximus, leviter flexuosus margine rotundato, ceteri duo acuti, posterior minor; dens anterior valve sinistrae maguns margine rotundato, supra exeavatus, antrorsum elongatus, posterior subperpendicularis, compressus, apice bifido, interdum conicus, debilis.

Alt. 13 ; long. 15 ; lat. $10^{1 / 2}$ mill.

Habitat in Camerunia: Ekumbi (S.).

Specimina 10, viventia collecta.

Cette espèce est beaucoup plus petite que Cyrenella (Cyrenoida) Dupontice Joannis, provenant du Sénégal; les dents sont, au contraire, très grandes relativement ì la coquille. Elle en diffère en outre par la conleur de rose dont sont teintés les crochets.

Par les 100 espèces et les 8 variétés mentionnées dans les pages qui précèdent, le nombre des espèces comnues de l'Afrique occidentale s'est accru de 35 espèces et de 1 variété. décrites pour la première fois. 


\section{Table alphabétique.}

Les synonymes sont en italique.

Achatina ampleore $\mathrm{JAY}_{\mathrm{AX}}$

Planche. Page

angustior (DOHRY)

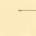

61

auripigmentum (REEVE) .

111

Aurora $\left(\mathrm{J}_{\mathrm{AY}}\right)$

balteata v. MARTENs

Barriana Sowerby

Calabarica Pfetffer

Camerunensis n. sp.

clavata GRAY

III, fig. 1-1 64

Downesii (GraY)

felina (ShutTLEWORTH)

Fraseri Pfeiffer . . . . . . . . . - 109

fuscidula MoneLeT . . . . . . . . . . . _ 106

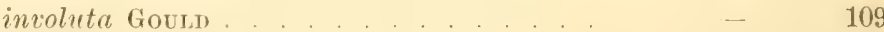

iostoma PFeifier

KnorTi JONAS

Leqiand Grateioup

marginata SWAINSON

> var. gracilior v. MaRTEes

Numidica (Reeve)

munum REEve

pulchella $\mathrm{v}$. Mnrtexs

rubicunda (SHUTTLEWORTH)

Shuttleworthi Preiffer

Sillimani Deshayes.

Smithi. Sowerby

Solimana (HoRELET)

striatella PHEIFFER

suturalis Phusipl

tenebrica (REEVE).

Vignoni Monelet 
BIHANG TILL K. SV. VET.-AKAD. HANDI. BAND 22. AFD. IV. N:0 2. 133

Auricula monile (Bruguì̀re)

I'anche. Page.

nitens LAMARCK

$-\quad 116$
$-\quad 118$

Bulimina Burnayi (DoHrN)

58

eminula (MORELET)

-
$-\quad 59$
-

Buliminus Burnayi DoHRN .

eminulus (MoRELET)

58

- 59

Bulimus Adansoni $\gamma$ PFeiffer

auripigmentum REEVE

83

Aurora $\mathrm{J}_{\mathrm{AY}}$

77

Burnayi (DoHRs)

58

Downesii (Gray)

sit

var. REEve

92

eminulus MoRELET

59

flammeus (MÜLLER)

75

Liberianus LAA.

monile Brugutìre

116

Numidicus ReEve

75

ovulus Bruguitere.

118

pupulus Moretat

;

Sillimani (Destriss)

82

Solimanus MoRELET

82

suffusus ReEve.

suturalis Pfetffer

82

tenebricus ReEve

74

Bulinus Downesii GraY

86

Cerithium muricatum BRUGUİ̀P:

121

Ovenii Férussac

121

Claviger Matoni (GRAY) .

122

Conoutuls flavus (GMeLIN)

116

pusillus (GMELIN)

118

Cyclophorus Preussi v. Martens

120

Cyrenella rosea n. sp.

V, fig. $24-30 \quad 131$

Dreissensia Holmi n. sp.

$\mathrm{V}$, fig. 17-23 130

Ennea Bonugolensis n. sp. .

1, fig. $24,27 \quad 21$

Bongeensis $n$. sp.

I, fig. $34-37$

10

Buchholzi v. Martens

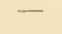

cavidens v. Martens

I, fig. $14-18$

columellaris v. Martens

romplicata $\mathrm{v}$. M.ARTENs

I. fig. 1!) 21

ronospira $v$. MARTENs

Dohrni E. A. SuIth

I, fig. 6-9 19

Duséni n. sp.

I, fig. 22-25 20

gemmia n. sp.

insignis $\mathrm{v}$. I

I, fig. 28-33

10

Liberiana (LEA). 
134 D'ATLLY, MOLLUSQUES TERRESTRES ET D'EAU DOUCE DE KAMÉROUX.

Ennea Yartensi n. sp.

Planche. l'ate.

Wartensi E. A. Sxiтu

- 15

monodon $\mathrm{v}$. MLARTENS

monodon MORELET .

$-\quad 12$

mucronata v. MARTENS

- 11

perforata n. sp.

1. tium. 11 1:; 16

pupula (MORELET)

serrata 11. sp.

1. $\mathrm{fi}$ :.. $38-41 \quad 17$

trigonostoma $\mathrm{v}$. MiARTENs.

12

'T'ullbergi n. sp.

I, $1 \mathrm{it}$. $4 \div, 43$

2:3

Excisa nov. sectio gen. Ennea .

Ganomidos n, gen.

Barrianum (Sowerbi)

III, fig. 5-10 70

Shuttleworthi (PFEIFFER) .

III, fig. 11-14 (69

Gibbus insignis (PeEIfFer)

Liberianus (LEA)

Martensi (E. A. Sumit)

forma minor $\mathrm{m}$.

pupulus (MORELET)

Glandina involuta (GoOLD) .

Glessula Calabarica (Penerfer)

clavata (GRAY)

Hapalus eminulus (MoReLET)

sulcatus n. sp. . .

III, fig $11-14$ (in

ร

4

9

ร

1ii!

$\begin{array}{ll}- & 11111 \\ - & 1011\end{array}$

-

$\mathrm{V}, \mathrm{fig} .5 \quad 60$

Helicarion columellaris n. sp.

II, tig. 1-S 31

depressus n. sp.

pertenuis n. sp.

, fig. $49-54 \quad 30$

plicatulus $v$. MARTENS

semimembranaceus $\mathrm{v}$. MARTENS

- $\quad 30$

I, fig. $44-48 \quad 34$

subglobosus n. sp.

II, fig. : $-14 \quad 33$

Helix Adansonice Morerat

Africana Pfeiffer.

Calabarica Pfeiffer .

calamechroa Joxds .

Camerunensis n. sp. .

1. fig. $11 \quad 13$

Folini Monelet

Ibuensis PFEIFFer

Jungneri n. sp.

V. fig. $11-11 ; \quad 5 i$

Liberice Brown

talcosa Gourn

tenera JoNAs.

troglodytes Morenet

$41 ;$

36

5) 1

istis

i)

$4 \%$

11

:iti

(j);

涪

:3t;

$10(1)$

1111

pileatus (v. MarTeNs)

105

retifer (v. MARTENS).

46

Hyatinia Adansonice (IoreLet)

Lanistes Bernardianus var. v. MaIrTexs. . . . . . . . . . 1:3

Libyeus (MoRELET)

$12: 3$ 
Lanistes Libycus var. Bernardianus (MoneLeT) . . .

Plarche, Page.

Limicolaria Aurora (JAY)

felina SHuttueworth

flammata PFEIFFER

flammea var. Numidica (REEve)

Kobelti n. sp.

-

123

Numidica (Reeve)

7
$-\quad 73$
$-\quad 75$

rubicunda ShutTueworth

suffusa (REEve)

suturalis (PFETFFER)

75

tenebrica (REEVE).

$-$

75

- 79

- 75

- 72

-

- 82

Macrochlamys calamechroa (Jonas)

- 74

Melampus flavus (GMeLiN)

38

Liberianus H. et A. ADAMS . . . . . . - 117

obovatus H. et A. ADAMS . . . . . . .

pusillus (GMELIN) . . . . . . . .

Melania foenaria REEve

117

- 118

fusca (GMelin) .

121

loricata REEVE.

Matoni GraY

$12 \%$

mutans GouLD.

123

nigrita MORELET

$12:$

Nigritina MoreLET

123

quadriseriata GRAY

121

tesselata LEA

121

123

Murex cingulatus GMELIN

fuscatus LINNÉ

123

fuscus GMeLiN

$\cdot 121$

radula LinNé.

$\begin{array}{ll}- & 121 \\ - & 122 \\ - & 121\end{array}$

$-\quad 121$

Nanina Adansonia (MoReLeT) . . . . . . . . . - 46

calamechroa (JoNAs) . . . . . . . . . _ -

Folini (MORELET) ….......... 47

troglodytes (MORELET) _ . . . . . . . -

Neritina Adansoniana RéClez . . . . . . . . . . . . . - 129

aquinoxialis Horelet . . . . . . . . _ - 127

Afra Sowerby . . . . . . . . . . . . _ _ 127

Africana RÉCLUZ ............. . - 127

Calabarica Mousson. . . . . . . . . _ 128

Listeri REEVE . . . . . . . . . . _ _ 127

Oweniana GRAY ............ . _ 126

rubricata MORELET . . . . . . . . . . _ - 128

Sangara MoRelet . . . . . . . . . . - n 129

Opeas egens n. sp. . . . . . . . . . . _

Orthalicus auripigmentum (REEVE) . . . . . . . - 83

Pedipes Dohrni n. sp............. - _ . 118

Perideris auripigmentum (REEVE) . . . . . . V fig. 3,4 , 83

Solimana (MoRELET) . . . . . . . . . . . E $\quad$ - 82

Vignoni (MORELET) . . . . . . . . . . - 84 
136 J'AITLY, MOLLTSOLES TERIESTRES ET D EAT DOTC'W IDE KAMEROTY.

Petitia petitia Joussenume.

Planche. I'age.

Petitia pulchella (v. Martens)

Potamides fuscatus (Linvé) i1

radula (LrNné)

\section{rathla (Lines)}

121

Psendachatina Dennisoni Pfriffrer. . . . . . . .

var. connectens $\mathrm{m}$.

IV, fig. 1-3 92

Downesii (GRAY)

var. grandinata (PFEIfFer)

IV, fig. $4,5 \quad k$ !

Downesii var. Sodeni KoBELT

grandinata PFEIFFER

Gravenreuthi BöTTGEP

var. Preussi Koneti .

Liljevalli n. sp.

Martensi n. sp.

Sodeni (КовецT)

- 90

- $\quad$ -

- $\quad 94$

- $\quad 14$

$\mathrm{V}$, fig. 1, 2 $9 \pi$

IV, fig. 7-9 (t)

IV, fig. 6 (4)

Pseudoglessula Calabarica (Pfeiffer)

clavata (GraY).

v var. Grayi m.

100

Duséni n. sp.

100

fuscidula (Monelet)

V. fig. \& 10107

involuta (GoULD).

10 ;

retifera (v. Martens).

109

Sjöstedti n. sp.

105

Psendotrochus Solimanus (MoRELET)

Pupa liberiana (LEA)

pupula (MORELET)

Rhachis Burnayi DoHrs

Rhysota Adansonioe (Morelex)

Calabarica (Pfeiffer)

Folini (MORELET)

Ibuensis (PFEIFFER)

percarinata (v. MaRTENs)

talcose (Gould)

tumidula (v. Martens) .

Stenogyra angustior. Domn

Calabarica (PFenfere)

fuscidula (Morelet) .

involuta (GovLD)

pileata v. Martens

retifera v. MARTENS

Y, fig. li. T 1114

Streptaxis Camerunensis n. sp.

Streptostele Bonjongoensis Truox

Buchholzi v. Martexs

mucronata (v. MLAR'TENS)

pusilla n. sp.

$\begin{array}{rr}- & 59 \\ - & i \\ - & 51 \\ - & 4 i \\ - & 54 \\ - & 4 i \\ & 41 \\ - & 45 \\ - & 53 \\ - & 41 \\ - & 100 \\ - & 106 \\ & 10 ! \\ - & 110 \\ - & 10 \%\end{array}$

1. fie. $1-5$

-- 24

- $\quad 25$

- $\quad 2 \%$

:6;

111

Subulina angustior DoHrN

clavata (Gisi)

109 
BIHANG TILL K. SV. VET.-AKAD. HANDL. BAND 22. AFD. IV. N:0 2. 137

Subulina involuta (GouLD)

Planche. Page

Succinea concisa MLoRELeT 109

spurca Gould .

114

Thapsia calamechroa (Jovas)

Sjöstedti n. sp. .

114

troglodytes (MoRELET)

II, fig. $15-20 \quad 39$

- $\quad 36$

Tralia pusilla (GMeciN) .

118

Trochomorpha Folini (Morelet) . . . . . . . . . . - - 47

Iluensis (PFETFER) . . . . . . . . . 41

talcosa (Gould) . . . . . . . . . . . . . . . . . . . . . . .

Trochonanina bifilaris DoHRN . . . . . . . . . . . . . . 52

Calabarica (PFetfFer) . . . . . . . - 54

Ibuensis (PEEIFEER) . . : . . . . . . 41

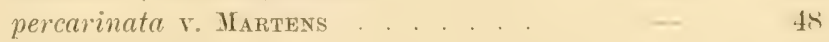

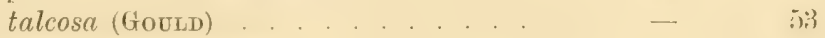

tumidula v. MARTENS . . . . . . $t 1$

Trochozonites Adansoniz (MoRELET) . . . . . . . . - . 46

bifilaris (DoriRN) . . . . . . . . II, fig. 49-55 52

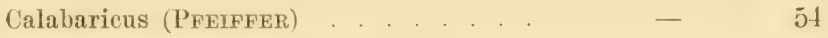

Folini (MORELET) . . . . . . . - 47

var. percarinatus (v. MaRnens) . $\quad-\quad 48$

hystrix I. sp.. . . . . . . . . . . . II, fig. $39-35 \quad 49$

Ibuensis (PFEIFFer) . . . . . . . . . . . $\quad$ - 11

var. tumidulus (v. Martens) . - $\quad 11$

Lindströmi n. sp. . . . . . . . . . . . II, fig. $40-42$ 4t

pilosus n. sp. . . . . . . . . . . . II, fig. $36-39 \quad 50$

reticulatus n. sp. . . . . . . . . . II, fig. $26-31 \quad 43$

suturalis n. sp. . . . . . . . . . . II, fig. $43-46 \quad 45$

talcosus (GovLD) . . . . . . . . . . . . . . . .

Théeli n. sp. . . . . . . . . . II, fig. 21 95 55

turbinatus n. sp. . . . . . . . . II, fig. 47, 48 il

Urocyclus Buchholzi v. Martens . . . . . . . . . . . $\quad 97$

Veronicella pleuroprocta v. MaIiTens . . . . . . . . . _ _ . 115

Voluta flava GMELIN . . . . . . . . . . . . . . . . . . 116

pusilla GMeLin . . . . . . . . . . . . . . . $\quad$ - 118

Xesta Buchholzi Bourguignat . . . . . . . . . . . . $\quad 36$

calamechroa (JonAs) . . . . . . . . . . . . . . . . 3r

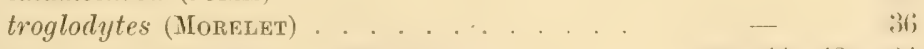

Zonitarion semimembranacens (v. MaRtens) . . . . . I, tig. $41-18$ it 


\section{Errata:}

I'. Y ligne 2 en descendant: au lieu de: Gibbus Jartensi E. A. Surtr. lisez: > $>$ (E. A. Smith).

- $\quad 6$ - $\quad$ - au lien de: Gibbus Martensi E. A. SмIтr. lisez: Ennea o

P. S5 ligne 33 en remontant: au lieu de: Pseudachatina Martensi m. lisez: $\quad$ Dennisoni PFEIFFer var. connectens m.

P. 90 ligne 3 en remontant: au lieu de: Pseudachatina Downesi KoBELT. lisez: > $\quad$ var. Sodeni KOBELT.

\section{Abréviations:}

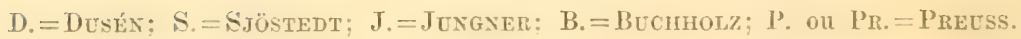





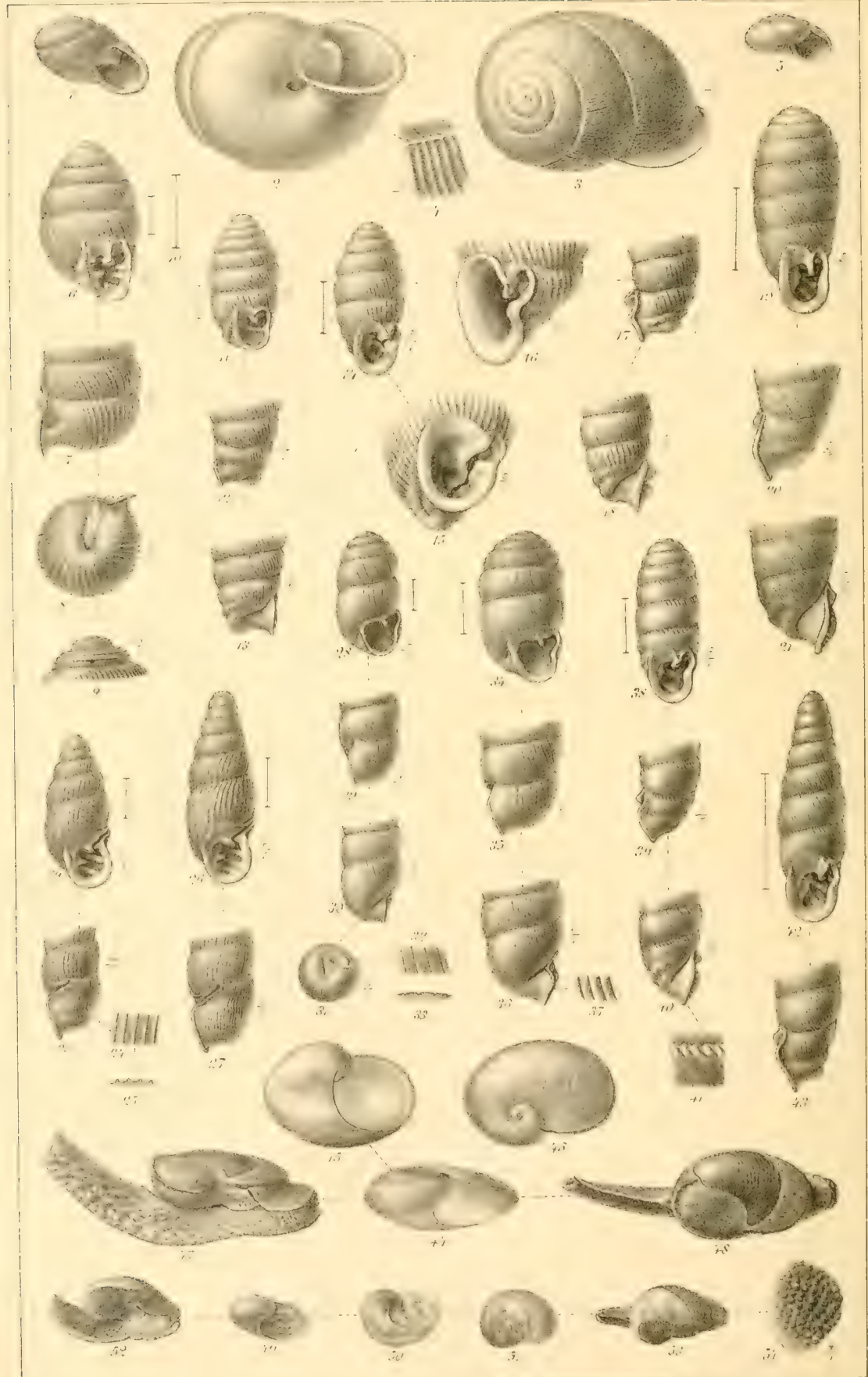

ज. Tiljevali cel \& lith 


\section{Planche I.}

Fig.

I'age.

$1-5$

Streptaxis Camerunensis n. $\mathrm{sp}$

6-9 Ennea conospira v. Martens forma minor m. . . . . 19

\begin{tabular}{|c|c|c|c|c|c|c|c|c|}
\hline 10 & 》 & $\gg$ & » & typica & . & . & & \\
\hline $11-13$ & $\gg$ & perforata n. sp. . . & . & . . . & . & . & & . \\
\hline $14-18$ & $\gg$ & columellaris v. Malten & . & . & . & . & & \\
\hline $19-21$ & $\gg$ & complicata v. Martens & . & . & . & . & & \\
\hline $22-25$ & $\gg$ & Duséni n. sp. . . . & . & . & . & . & & \\
\hline $26--27$ & ;) & Boangolensis n. sp. . & . . . & . & . & . & & . \\
\hline $28-33$ & $\gg$ & gemma n. sp. . . . & . & . & . & . & & . \\
\hline $34-37$ & $\gg$ & Bongeensis n. sp. . & . . . & . & . & . & & \\
\hline $38--41$ & $\Rightarrow$ & serrata n. sp. . . . & . . . & . & . & . & & . \\
\hline $42, \quad 43$ & $\gg$ & Tullbergi n. sp. . . & - . & . . & & . & & \\
\hline $44-48$ & Zoni & ion semimembranaceus & (v. M & ARTENS) & & . & & . \\
\hline $49-54$ & Hel & $\begin{array}{l}\text { ion pertenuis n. sp.; } \\
\text { ties de l'animal renferm } \\
\text { dées pour conserver } \\
\text { ie du manteau. . }\end{array}$ & dans & les fig. & & & & \\
\hline
\end{tabular}




\section{Planche II.}

Fig.

Page.

$1-8$ Helicarion columellaris n. sp.; fig. 6: sculpture embryonnaire; fig. 8: partie du manteau, . , 31

9-14 subglobosus n. sp.; fig. 13: partie du manteau 33

$15-20$ Thapsia Sjöstedti n. sp. . . . . . . . . . . 39

21 -... Trochozonites Théeli в. sp. . . . . . . . . . . 55

2 2 $: 1$ reticulatus n. $\mathrm{sp}$.

$\therefore 2$ hystrix n. sp. . . . . . . . 49

3i-:3 pilosus n. sp. . . . . . . . 50

$40-42$ Lindströmi n. sp. ......... 44

$43-46$ suluralis u. sp. . . . . . . . . ti;

47. $48 \quad>\quad$ turbinatus n. sp. . . . . . . 51

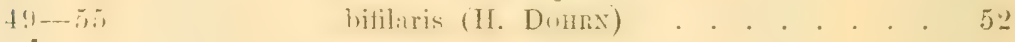


Bihang till K.Vet. Akad. Handl. Bd.22. Afd IV.N: z.

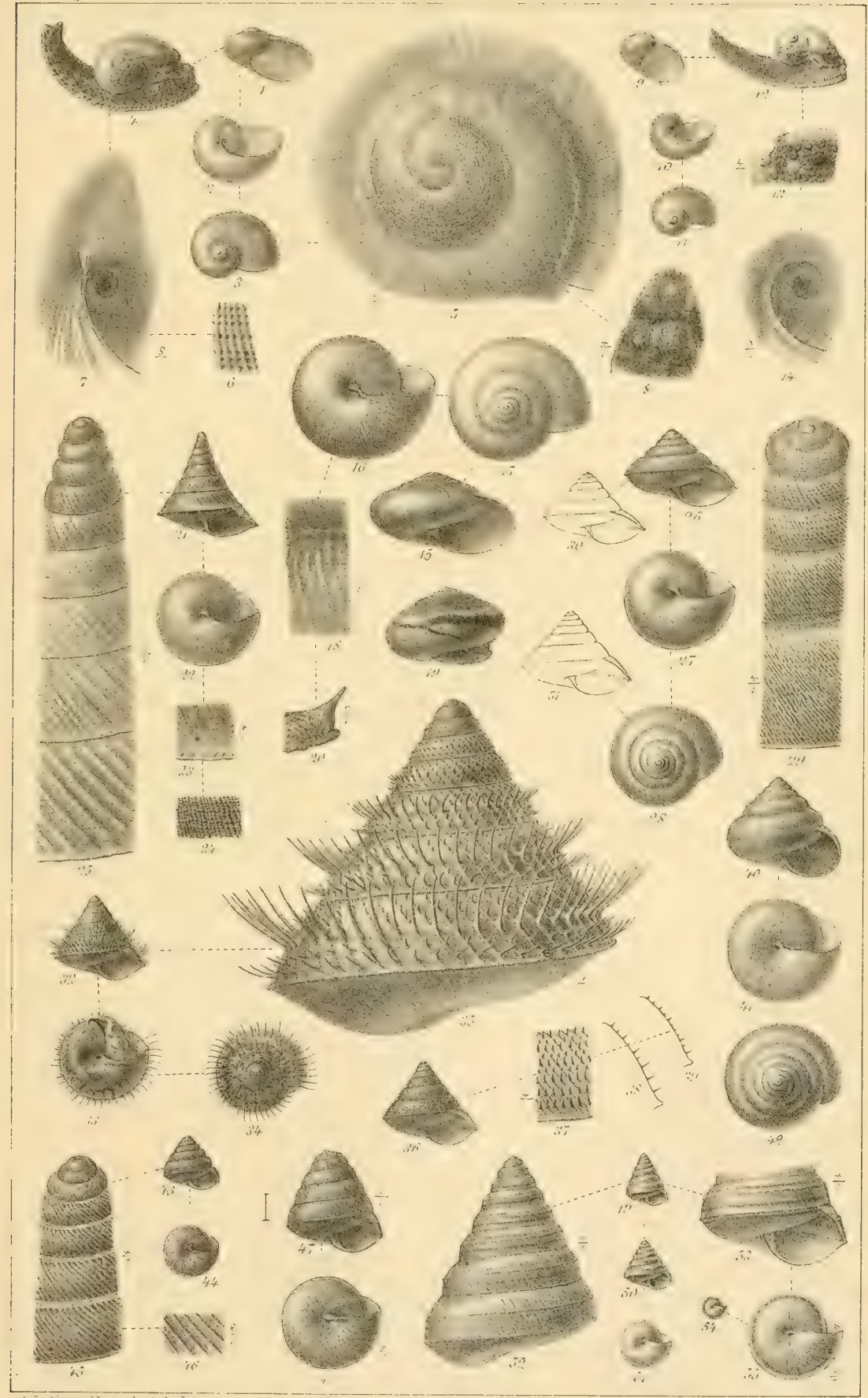






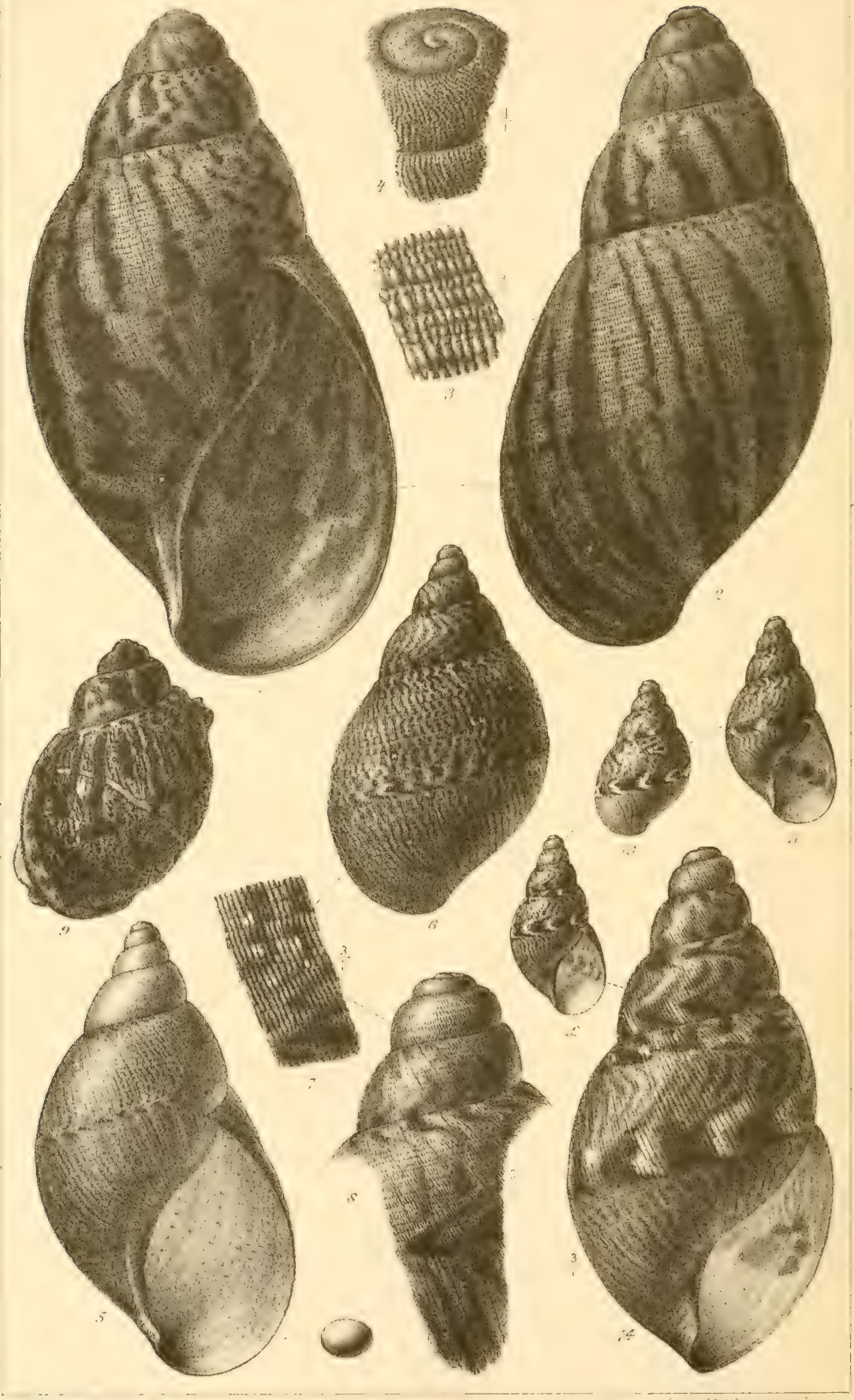




\section{Planche III.}

Fig.

Page.

$\begin{array}{ll}1-4 & \text { Achatina Camerunensis n. sp. . . . . . . . . . } \\ 5-10 & \text { Ganomidos. Barrianum (Sow }\end{array}$ un animal retiré de la coquille et conservé dans l'alcool . . . . . . . . . . . 70

11-14 Ganomidos Shuttleworthi (Pfeiffer) . . . . . . 69 


\section{Planche IV.}

rie.

Page.

1--.) Pseulachatina Dennisoni Pfeifrer var. connectens m.; la fig. 2 représente un jeune exemplaire vu de la base . . . . . . . . . 92

4. 5 D Downesii var. grandinata (PFerfFer) . . . 89

(i) Sodeni (KоBEIT) . . . . . . . 90

7--! Martensi n. sp.; la fig. 9 représente un jeune exemplaire vu de la base... . 95 


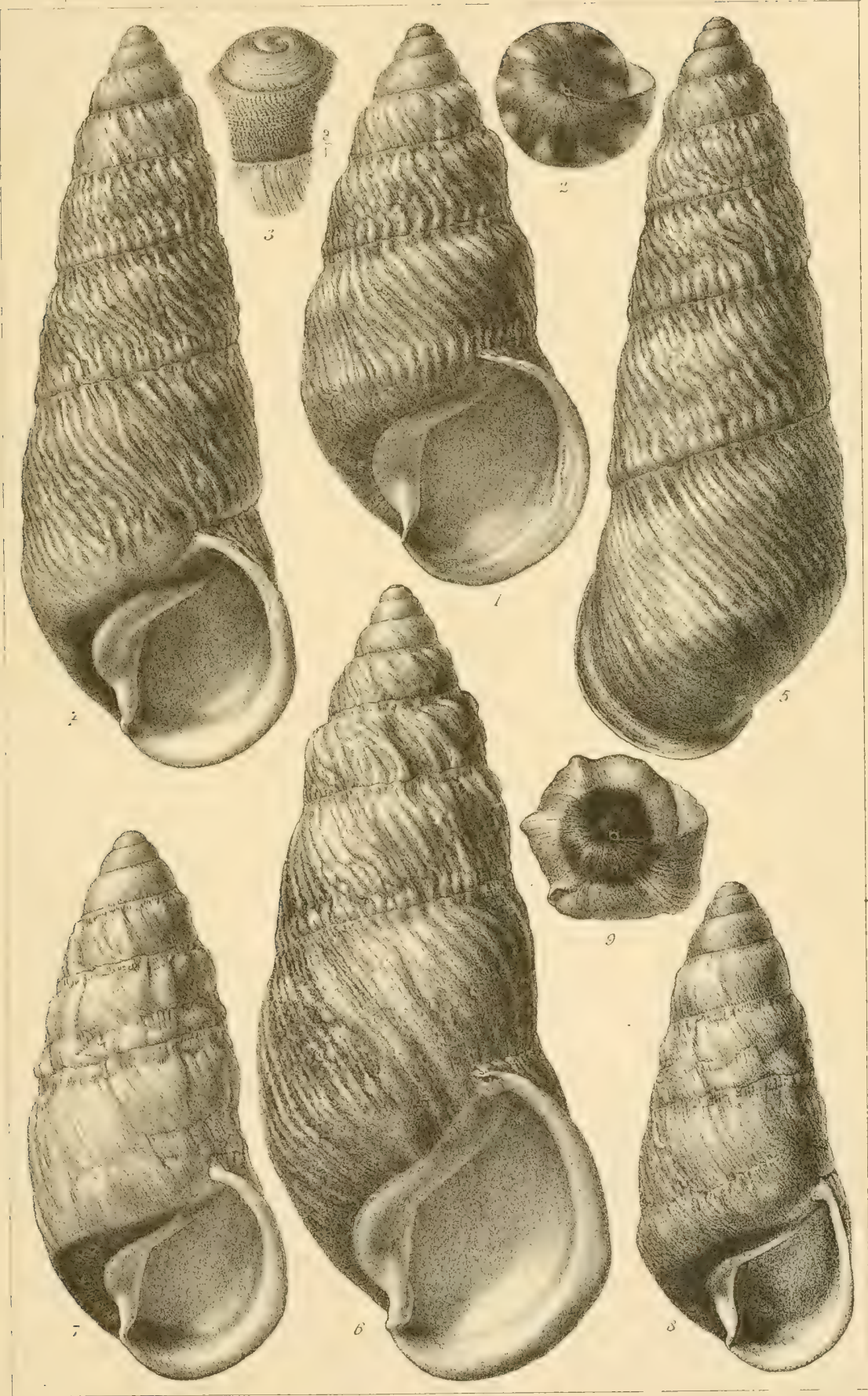



Planche V. 
Fig.

Page

1, 2 Pseudachatina Liljevalli n. sp. . . . . . . . . . 98

3,4 Perideris auripigmentum (REeve) . . . . . . . . 83

$5 \quad$ (?) Hapalus sulcatus в. sp. . . . . . . . . . . 60

6, 7 Pseudoglessula Sjöstedti n. sp. . . . . . . . . 104

8-10 $\gg$ Duséni n. sp. . . . . . . . 107

$11-13$ Helix Camerunensis n. sp. . . . . . . . . 57

$14-16 \gg$ Jungneri n. sp. . . . . . . . . . . . . 57

17-23 Dreissensia Holmi n. sp.; fig. 23: vue oblique pour faire voir la lamelle du septum . . . . . . . . 130

24-30 Cyrenella rosea n. sp.; la fig. 28 représente un animal retiré de la coquille et conservé daus l'aleool . . . 131 
Bihang till K.Vel. Akad. Handl. Bd. 22. Afd. TV. №\&.

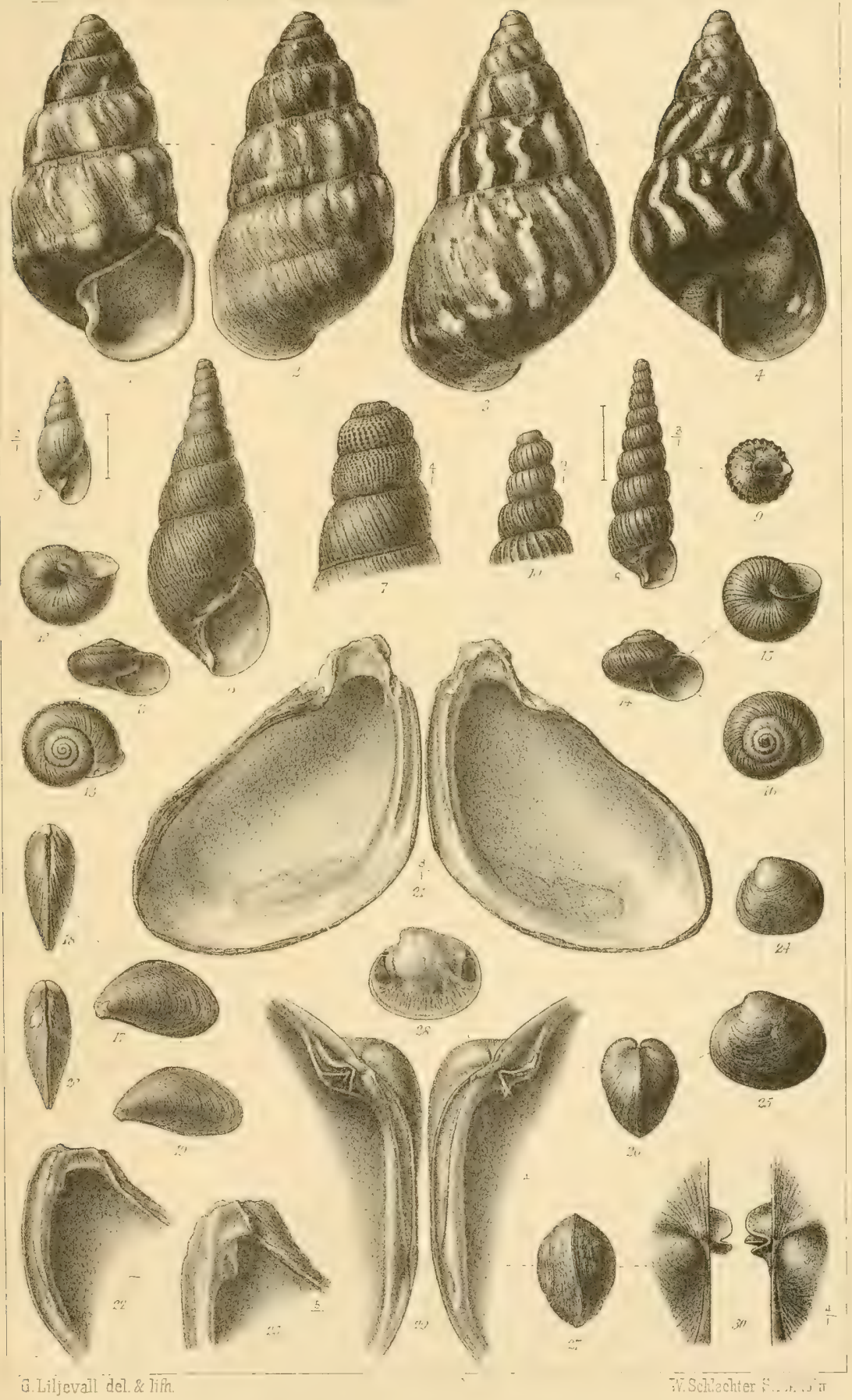










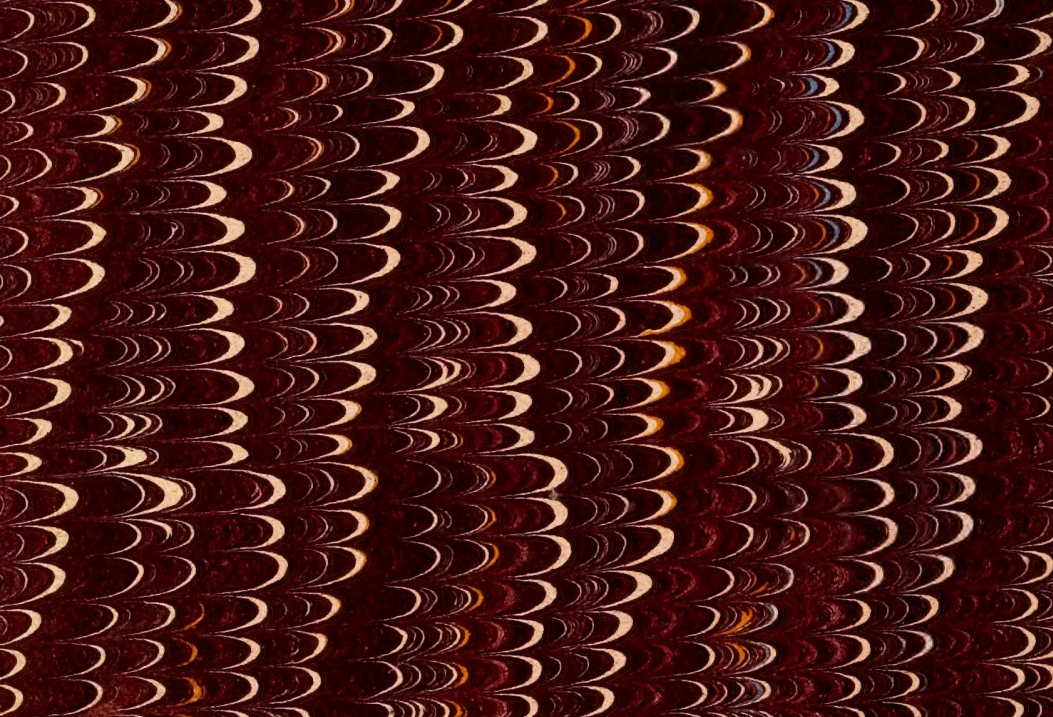
333
अ) 3
320035

उ) 310

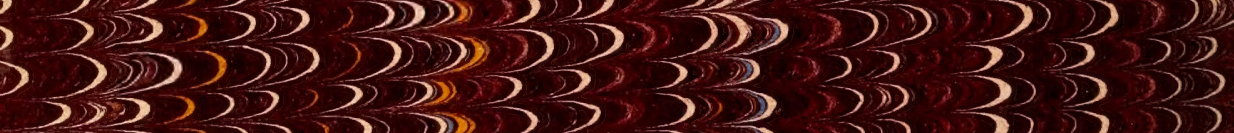

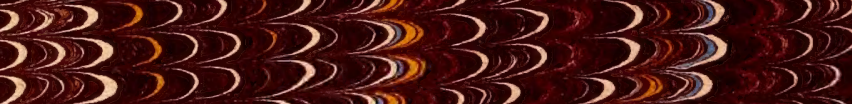
( 53,35035 3) $3,3>3>3>3$

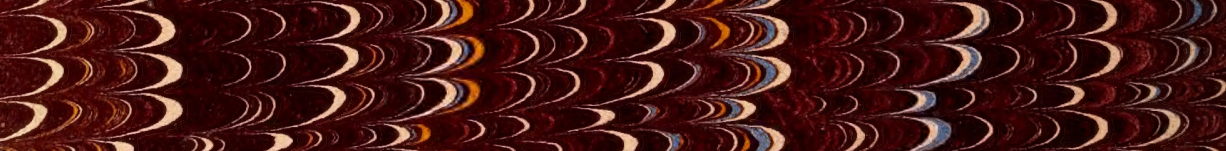
3. D () . , 3 () ()) 》 (7) 33 , 3,3 135

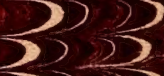



